JHU/APL

ANSP-M-19

DOE/OSA/32087-19

OCTOBER 1988

Copy No. OB?

\section{Ronguad ky Com}

MAR 011989

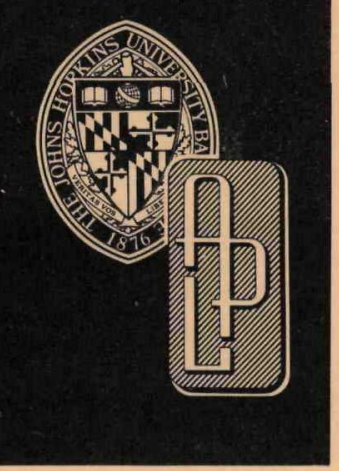

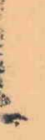

Aerospace Nuclear Safety Program

\title{
REENTRY RESPONSE OF THE LIGHT WEIGHT RADIOISOTOPE HEATER UNIT RESULTING FROM A VENUS-EARTH-EARTH GRAVITY ASSIST MANEUVER ACCIDENT
}

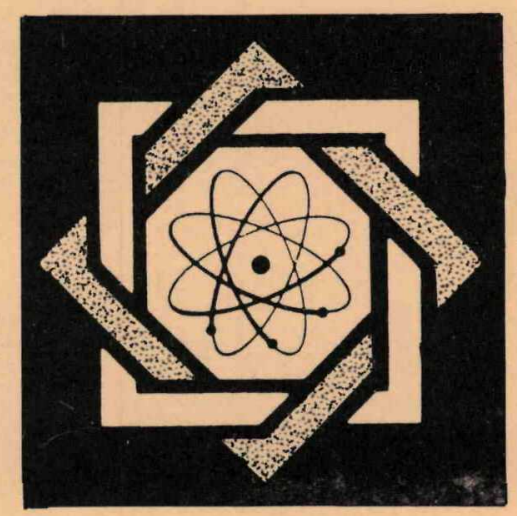

THE JOHNS HOPKINS UNIVERSITY • APPLIED PHYSICS LABORATORY

DO NOT MICROFILM COVER 


\section{DISCLAIMER}

This report was prepared as an account of work sponsored by an agency of the United States Government. Neither the United States Government nor any agency Thereof, nor any of their employees, makes any warranty, express or implied, or assumes any legal liability or responsibility for the accuracy, completeness, or usefulness of any information, apparatus, product, or process disclosed, or represents that its use would not infringe privately owned rights. Reference herein to any specific commercial product, process, or service by trade name, trademark, manufacturer, or otherwise does not necessarily constitute or imply its endorsement, recommendation, or favoring by the United States Government or any agency thereof. The views and opinions of authors expressed herein do not necessarily state or reflect those of the United States Government or any agency thereof. 


\section{DISCLAIMER}

Portions of this document may be illegible in electronic image products. Images are produced from the best available original document. 
Th1s work was prepared as an account of work sponsored by an agency of the United States Government. Ne1ther the Un1ted States Government nor any agency thereof, nor any of their employees, makes any warranty, express or Implled, or assumes any legal liabllity or responsibllity for the accuracy, completeness, or usefulness of any Information, apparatus, product or process disclosed, or represents that 1 ts use would nor. 1nfringe privately owned rights. References herein to any specific commerical product, process, or service by trade name, trademark, manufacturer, or otherwise, does not necessarily constitute or $1 \mathrm{mply} 1 \mathrm{ts}$ endorsement, recommendation, or favoring by the Unfted States Government or any agency thereof. The views and opinions of authors expressed herein do not necessarily state or reflect those of the Un1ted States Government of any agency thereof.

\section{DO NOT MICROFILM COVER}


JHU/APL

ANSP-M-19

DOE/OSA/32087-19

OCTOBER 1988
ANS P-M- -19

DE89 005359

\section{REENTRY RESPONSE OF THE LIGHT WEIGHT RADIOISOTOPE HEATER UNIT RESULTING FROM A VENUS-EARTH-EARTH GRAVITY ASSIST MANEUVER ACCIDENT}

\section{DISCLAIMER}

This report was prepared as an account of work sponsored by an agency of the United States Government. Neither the United States Government nor any agency thereof, nor any of their employees, makes any warranty, express or implied, or assumes any legal liability or responsibility for the accuracy, completeness, or usefulness of any information, apparatus, product, or process disclosed, or represents that its use would not infringe privately owned rights. Reference herein to any specific commercial product, process, or service by trade name, trademark, manufacturer, or otherwise does not necessarily constitute or imply its endorsement, recommendation, or favoring by the United States Government or any agency thereof. The views and opinions of authors expressed herein do not necessarily state or reflect those of the United States Government or any agency thereof.

THE JOHNS HOPKINS UNIVERSITY — APPLIED PHYSICS LABORATORY Johns Hopkins Road, Laurel, Maryland 20707

Operating under Contract N00039-87-C-5301 with the Department of the Navy

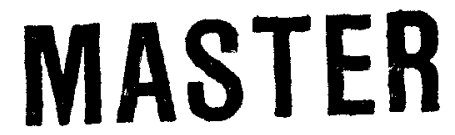


REENTRY RESPONSE

OF THE LIGHT WEIGHT RADIOISOTOPE HEATER UNIT

RESULTING FROM A VENUS-EARTH-EARTH GRAVITY ASSIST

MANEUVER ACCIDENT

Approved by

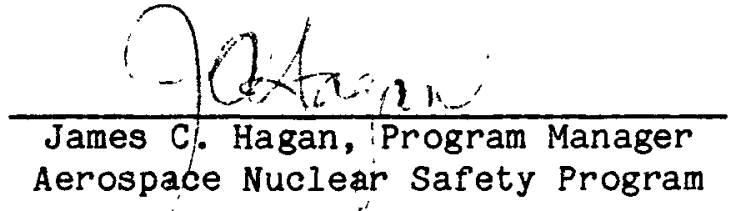

October 1988 


\title{
LWRHU REENTRY RESPONSE
}

\author{
The Johns Hopkins Uni versity \\ Applied Physics Laboratory
}

\section{ABSTRACT}

Reentry analyses consisting of ablation response, thermal response and thermal stress response have been conducted on the Light Weight Radioisotope Heater Unit for Galileo/VEEGA reentry conditions.

Sequential ablation analyses of the LWRHU aeroshell, the fuel clad, and the fuel pellet have been conducted in reentry regimes where the aeroshell has been deemed to fail. The failure criterion for ablation is assumed to be recession corresponding to $50 \%$ of the wall thickness (the design criterion recommended in the DOE overall Safety Manual). Although the analyses have been carried far beyond this limit (as presented and discussed herein), JHU/APL endorses the position that fallure may occur at the time that this recession is achieved or at lower altitudes within the heat pulse considering the uncertainties in the aerodynamic, thermodymamic, and thermo-structural analyses and modelling. These uncertainties result mainly because of the high energies involved in the VEEGA reentries compared to orbital decay reentries. Risk evaluations should consider the fact that for shallow flight paths the unit may disassemble at high-altitude as a result of ablation or may remain intact until it impacts with a clad that had been molten.

Within the limitations of the methodologies and assumptions of the analyses, the results indicate that:

1. For side-on reentry and shallow reentry angles $\left(\gamma \leq\left|-40^{\circ}\right|\right)$ LWRHU aeroshell ablation failure could result. The shallower the angle, the more probable is the failure.

Following side-on entry failure, the LWRHU clad is expected to melt within one second and release the fuel pellet. This occurs at altitudes greater than about $90,000 \mathrm{ft}$. Significant melt of the fuel pellet could occur following pellet release.

2. For side-on reentry at steep angles, neither ablation failures nor catastrophic thermal stress failures of the LWRHU are expected to occur.

3. For end-on entry, neither ablation failures, thermal failures nor catastropic thermal stress failures were predicted for the range of flight path angles between $-10^{\circ}$ and $-90^{\circ}$. Extrapolation of ablation predictions to more shallow flight path angles indicates probable ablation failure and fuel release for angles less than about 8 degrees. 
Both angle of attack orientations analyzed are ideal. The orientation at hypersonic speeds and during the heat pulse interval may be either somewhere in between or random tumbling.

4. Successful ablation response tests were completed for the LWRHU (and other modules) at conditions approximating orbital decay entries. The data have not been analyzed sufficiently to provide results at this writing. Succeeding tests are planned at higher pressure and enthalpy conditions.

In the case of shallow angle reentry for the side-on stable orientation, another scenario for fallure is that in which the aeroshell does not come apart even though burn-through occurs. For this scenario the following impact configurations may be possible in the reentry zones defined in this study as: Zone A (reentry angle, $\left.\gamma \leq\left|7^{\circ}\right|\right)$; Zone $B\left(\left|7^{\circ}\right| \leqslant \gamma \leqslant\left|20^{\circ}\right|\right)$; and, Zone C $\left(\left|20^{\circ}\right| \leq \gamma \leq\left|40^{\circ}\right|\right)$.

Zone A: Full LWRHU assembly with aeroshell burn-through on windward surfaces leading to direct exposure of underlying PG sleeves, extensive melting and resolidification of clad.

Zone B: Full LWRHU assembly with partial burn-through of aeroshell at the ends with direct exposure of underlying PG insulator end plug, possibly partial clad meltings and resolidification in the near end regions.

Zone C: Full LWRHU assembly with partially ablated aeroshell clad assembly intact.

In assessing the radiological risk, both failure scenarios (ablation leading to release of the fuel pellet an containment of a melted clad) should be considered as possible.

The analyses included in this report concentrate on VEEGA reentry scenarios. Analyses reported previously have demonstrated that the LWRHU has adequate design margin to survive reentry from orbital decay scenarios and most injection scenarios at speeds up to escape speeds. The exception is a narrow range of flight path angles that produce multiple skip trajectories which may have excessive ablation. 


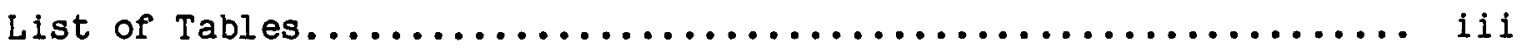

List of Figures................................ v

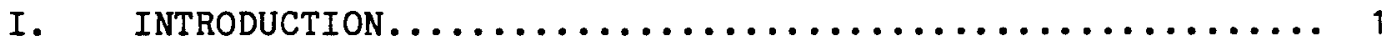

II. MODULE DESCRIPTION ........................ 2

A. Geometry and Mass Properties................ 2

B. LWRHU Reentry Design Features................ 2

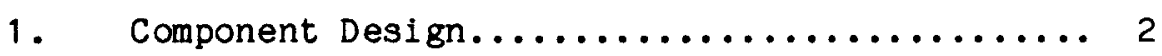

2. System Design..................... 3

III. REENTRY CONDITIONS ....................... 4

IV. GENERAL DESCRIPTION OF ANALYSIS................ 5

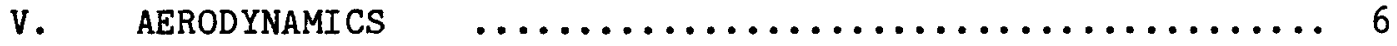

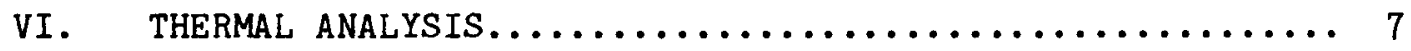

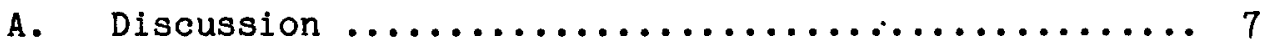

1. Reentry Design Evaluation Versus Design Development .................. 7

2. VEEGA Reentry Safety Evaluation Process Guidelines...................... 7

B. Assumptions........................... 7

1. Initial Reentry Conditions............. 7

2. Reentry Configurations ................. 8

3. Reentry Orientation.................. 8

4. Failure Criteria for Ablation and Thermal Response.................. 8

5. Breakup Criteria................... 9

C. Methodology - Side-on Analyses................ 9

1. Zonal Approach for VEEGA Reentry Safety Evaluation .................. 9

2. Reentry Trajectory Analyses Side-on Stable LWRHU ................ 10

3. Engineering Methods and Correlations........ 12

4. Summary of Zonal Environments Side-on LWRHU Reentry Configuration....... 14 


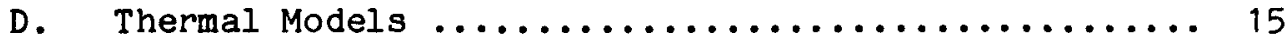

1. Thermal and Ablation Response

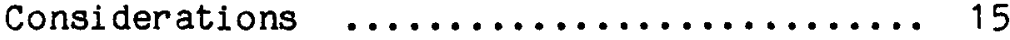

E. Results of Analysis - Side-on Stable

Response Behavior.................. 22

1. Reentry Configuration: Side-on

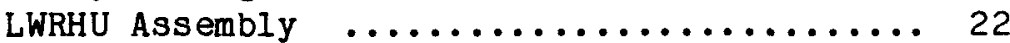

2. Reentry Configurations: Side-on

Stable Clad/Fuel.................... 23

3. Bare Fuel Pellet Side-on Reentries.......... 24

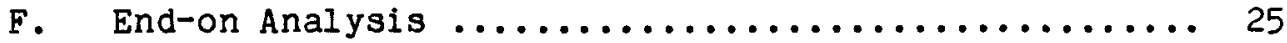

1. 3DOF Trajectory and other Input

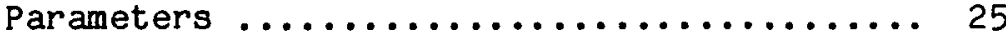

2. Results of 2-D End-on Thermal Analysis....... 25

G. Comparison of Side-on and End-on

Reentry Ablation Response................. 28

VII. THERMO-STRUCTURAL ANALYSIS $\ldots \ldots \ldots \ldots \ldots \ldots \ldots \ldots \ldots \ldots \ldots$

A. Summary and Conclusions .................. 31

B. Methodology and Assumptions................. 32

C. Results of Side-On Stable Analysis............. 36

D. Results of End-on Stable Analysis............. 39

VIII. LWRHU ABLATION RESPONSE TESTS.................. 39

A. Test Program ........................ 40

B. Thermal Response Predictions for 60

Ablation Tests at NASA/Ames in the $20 \mathrm{MW} \ldots \ldots \ldots 40$

IX. OTHER REENTRY CONDITIONS.................... 43

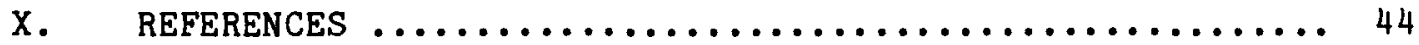


Table 1 Estimated Drag Coefficients for Light Weight Radioisotope Heater Unit

Table 2 Estimated Drag Coefficients and Terminal Velocity for the LWRHU

Table 3 Guidelines for LWHRU Reentry Evaluation Process - Galileo/VEEGA

Table 4 Galileo-VEEGA Reentry Trajectory Analysis Conditions, Assumptions and Limitations

Table 5 Galileo-VEEGA Reentry Environment Engineering Methods for Side-on Stable LWRHU Configuration

Table 6 Galileo-VEEGA Reentry Environmental Parameters LWRHU Side-on Stable Attitude

Table 7 Initial Radial Temperature Distribution for Side-on Stable LWRHU Reentries

Table 8.a Thermal Properties for FWPF carbon-carbon (Table C-1-2, Ref. 56)

Table 8.b Thermal Properties of Pyrolytic Graphite; C-plane (LWRHU radial) direction (Table G-6-1, Ref. 56)

Table 8.c Thermal Properties for Pyrolytic Graphite, A-B Plane (LWRHU Circumferential) Direction (Table G-2-3, Ref. 56)

Table 8.d Thermal Properties for Pt30Rh (Table P-2-1, Ref. 56)

Table 8.e Thermal Properties for Plutonia (Table 3, Ref. 55).

Table 8.f Thermal Properties for Plutonia, heat of Fusion Ef fect Incorporated at Melt Failure Threshold ( $\mathrm{T}_{\text {melt }}{ }^{-500}$ )

Table 9 Galileo/VEEGA Reentry Release Conditions Clad/Fuel Assembly

Table 10 Galileo-VEEGA Reentry Release Conditions for the Bare Fuel Pellet

Table 11 LWHRU End-On Reentry Analysis Initial (Inertial) Conditions for 3DOF Trajectory Anal ysis

Table 12 LWRHU Internal Contact Conductances 
Table 13 LWRHU Side-On Stable Coarse Model VEEGA Steep Reentry Hoop and Axial Stresses and Safety Factors

Table 14 LWRHU Side-On Stable Fine Model VEEGA Steep Reentry Hoop and Axial Stresses and Safety Factors at 1.40 Seconds - Basic Assumptions

Table 15 LWRHU Side-On Stable Fine Model VEEGA Steep Reentry Hoop and Axial Stresses and Safety Factors at 1.40 Seconds - Refined Assumptions

Table 16 LWRHU End-On Stable VEEGA Steep Reentry Hoop and Radial Stresses and Safety Factors (Elastic Tensile Material Properties) 


\section{Figures}

Figure 1 Cross-sectional view of LWRHU assembly (from Reference 2)

Figure 2 LWRHU aeroshell body (reproduced from Reference 2)

Figure 3 LWRHU clad body (reproduced from Reference 2)

Figure 4 LWRHU fuel pellet (reproduced from Reference 2)

Figure 5 Varlation of initial altitude versus flight path angle for maximum and expected initial velocities for discrete GPHS module and LWRHU reentries; Galileo-VEEGA mission profile (from Reference 5)

Figure 6 a. Typical initial reentry velocity - flight path angle map; negligible shock layer radiation

b. Characteristic convective heat pulse for various heat source reentry design corridors (from Reference 9)

Figure 7 Zonal evaluation of LWRHU reentry performance for Galileo/VEEGA; side-on stable LWRHU assembly reentry configuration

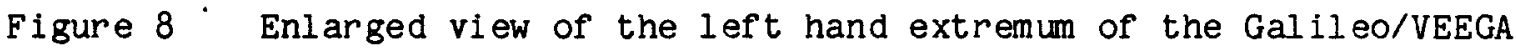
reentry map; side-on stable LWRHU assembly reentry configuration

Figure 9 Representative Galileo-VEEGA trajectory profiles for Zone A through E; hypersonic flight period for side-on stable LWRHU reentry configuration

Figure 10 Aerothermodynamic regimes for the Galileo-VEEGA zonal reentries; side-on stable LWRHU configuration

Figure 11 a. The effect of mass transfer and chemical species on surface radiation and convective heat transfer for superorbital earth reentry conditions (Ref. 17)

b. Blowing-nonblowing heat transfer ratio $(\psi)$ as a function of the mass transfer parameter, $B^{\prime}$. (Ref. 43)

Figure 12 Composite plot comparing experimental data with the Detra, Kemp and Riddell correlation

Figure 13 Windward laminar boundary layer convective heating rate distribution

Figure 14 Leeward convecting heating rate distributions around a sphere (Ref. 30) 


\section{Figures (cont)}

Figure 15 Smoothed values of experimental heat transfer distribution around a circular cylinder (Ref. 32)

Figure 16 Stagnation velocity gradient correction on the side-on LWRHU stagnation convective heating rate (without geometry scaling)

Figure 17 a. 2-D thermal model for side-on LWRHU assembly; mid-span cross section (not to scale)

b. 2-D thermal model for side-on clad/fuel assembly (not to scale)

c. 2-D thermal model for side-on fuel pellet (not to scale)

Figure 18 Surface energy balance-reentry thermal analysis

Figure 19 Surface mass balance-reentry thermal analysis

Figure 20 Thermochemical ablation regimes for graphite

Figure 21 Aeroshell total stagnation recession predictions; side-on stable LWRHU assembly reentry configuration

Figure 22 Clad stagnation maximum and impact temperatures (assuming no prior ablation or thermal stress failure events); side-on stable LWRHU assembly reentry conf iguration

Figure 23 Clad thermal response behavior subsequent to aeroshell failure (Fig. 21) and release; side-on stable clad/fuel assembly reentry configuration

Figure 24 Estimate of mass fraction of fuel melt resulting from prior sequential failure of the LWRHU; side-on stable bare fuel pellet reentry configuration

Figure 25 LWRHU stagnation reentry heating

Figure 26 Sketch of the end-on LWRHU thermal model which shows the 762 nodal points. Dimensions are in inches in this particular drawing.

Figure 27 Heating boundary conditions used in the APL analyses

Figure 28 LWRHU aeroshell windward end-face and clad temperature response, Galileo-VEEGA steep ( -90 degree) end-on reentry orientation 
Figure 29 LWRHU aeroshell windward end-face and clad temperature response, Galileo-VEEGA shallow ( -10 degree) end-on reentry orientation

Figure 30 LWRHU end-on ablation profile, Galileo-VEEGA, -90 degree reentry

Figure 31 LWRHU end-on ablation profile, Galileo-VEEGA, -10 degree reentry

Figure 32 Comparison of the aeroshell maximum recession predictions for side-on and end-on LWRHU assembly reentry configurations

Figure 33 LWRHU aeroshell, dimensions

Figure $34 \quad$ Boundary conditions - side stable models

Figure 35 Coarse mesh planar cross section model for side-on reentry calculations

Figure 36 LWRHU nodal temperature distributions

Figure 37 LWRHU coarse model hoop stress distributions

Figure 38 Fine mesh planar cross section model for side-on reentry calculations

Figure 39 Boundary conditions - end stable models

Figure 40 Axisymmetric model for end-on reentry calculations

Figure 41 Heat pulse of an orbital decay earth reentry for a LWRHU with the end-on orientation

Figure 42 Aeroshell recession at the stagnation point of the LWRHU for an orbital decay reentry with the end-on stable orientation

Figure 43 Total pressure history of an orbital decay reentry for an LWRHU with the side-on stable orientation

Figure 44 Heat transfer parameter $\left(\dot{q} / H_{T}\right)$ history for the LWRHU for an orbital decay reentry with the side-on stable orientation

Figure 45 End-on stagnation point temperature history for the LWRHU -- 20MW, AHF, 12" nozzle

Figure 46 Aeroshell recession at the end-on stagnation point for the LWRHU -- 20MW, AHF, 12" nozzle 
THE JOHNS HOPKINS UNIVERSITY APPLIED PHYSICS LABORATORY LAUREL MARYLAND

I. INTRODUCTION

In the Aerospace Nuclear Safety Program (ANSP) effort associated with the Galileo Mission, The Johns Hopkins University/Applied Physics Laboratory (JHU/APL) has been charged by the Department of Energy (DOE) (Reference 1) with the primary responsibility of assessing the survivability of the Light Weight Radioisotope Heater Unit (LWRHU) in an inadvertent entry into the earth's atmosphere. This report constitutes the JHU/APL contribution to the Final Safety Analysis Report (FSAR) for the LWRHU. It includes methodology, assumptions, and results of thermal, thermal response, and thermal stress analyses. Some preliminary observations from ablation response tests are also included.

The key personnel and their roles in this study are as follows:

J. C. Hagan is the Program Manager for the APL/JHU Aerospace Nuclear Safety Program; E. F. Lucero is the Assistant Program Manager; P. T. Brenza: Pre-test Thermal Response Predictions and Fuel Simulant Analyses; C. C. Chan: Thermal and Thermal Response Predictions - End-On; D. W. Conn: Thermal and Thermal Response Predictions - Side-On and Sequential Breakup; Design Rationale and Evaluation Processes; Thermal Analyses Code Development; R. A. Drobnick: Test Module Holder Design; J. A. Ecker: Thermal Stress Analyses; E. F. Lucero: Aerodynamics; S. A. Lutz: Test Program Plan and Test Engineer for Arc Jet Ablation Tests; and, S. M. Remeikis: program secretary. 


\section{MODULE DESCRIPTION}

\section{A. Geometry and Mass Properties}

The geometric parameters and mass properties that are pertinent to the aerodynamic, thermal, and thermo-structural analyses of the LWRHU and components, Figures 1-4, are described here for easy reference. The materials are shown in Figure 1; the dimensions of the various components (Figures 2 to 4), are given in Appendix B.1 of Reference 2; the weights are from Table B. 1 of Reference 2. A description of the design rationale follows for completeness.

\section{B. LWRHU Reentry Design Features}

The basic elements of an effective reentry design are demonstrated in Figure 1 which is a cross sectional view of the LWRHU assembly. For radioisotope devices, there are generally four such elements, three of which relate to component design and one to overall system considerations.

\section{Component Design}

The three component design elements are the heat shield member, thermal insulation member(s) and the structural containment member. For the LWRHU design, the heat shield (aeroshell) member is fabricated using a 3-D carbon-carbon (FWPF ${ }^{\text {tm }}$, Avco Corp.) which maintains the high temperature heat accommodation and ablation performance attributes identified with past generation graphites (e.g., the $\mathrm{POCO}^{\mathrm{R}}$ grades) but most importantly, possesses superior thermo-structural capabilities. This is particularly the case regarding fracture mechanics as related to crack propagation arrestment. The design's thermal insulation is provided by five members, all of which are manufactured from pyrolytic graphite (PG). For the insulator plugs, PG's low thermal conductivity (' $c$ ') direction is aligned in the longitudinal direction to thermally protect the clad against end-on orientation reentries. Similarly, the ' $c$ ' direction is aligned radially for the nested tubes to provide protection against side-on reentries. Standoffs have been machined on the tubes and clad to provide gaps at the radial interfaces to further increase thermal resistance in this direction. To prevent the formation of critical thermal paths to the clad along PG's high conductivity direction (orthogonal to the ' $c$ ' direction), the tubes and end plugs are mated in a stepwise configuration. This stepped interface also prevents radiation 'shine' directly to the clad from the aeroshell's interior surface. Finally, the structural component (clad) is fabricated from Pt30Rh alloy which meets the required fuel compatibility requirements, has high temperature capability (a carbon eutectic melting point of $3660^{\circ} \mathrm{R}$ ) and excellent ductility behavior at room temperatures and above. It is this last attribute which permits significant absorption of hard target impact energies via strain deformation without the incurrence of rupture and subsequent fuel release. 


\section{System Design}

System design relates to size, form and weight which, when taken collectively, dictates a body's reentry ballistic coefficient. A low ballistic coefficient configuration, as of ered by the LWRHU, results in high decelerations and consequently, reduced heat pulse flight periods, reduced heating rates (for a fixed aerodynamic configuration) and reduced time integrated heat loads. Additionally, the lowering of a body's ballistic coefficient will prolong the subsonic cool down flight period and reduce impact velocities, both being important considerations related to clad impact performance. With regard to size, a small reentry body such as the LWRHU provides numerous advantages beyond its effect on ballistic coefficient. For example, a small size will (a) inhibit boundary layer transition and the advent of turbulent heating (b) form a thin shock layer with reduced shock layer heating (an important factor for VEEGA generated entries) and (c) permit ground testing at full scale in a variety of ground test facilities. However, a diminutive size also has negative effects such as increased convective heating rates for stable reentries and 11 mited system thermal capacitance provided by the heat shield and internal components. It is this latter consideration, particularly when further aggravated by a design requirement of low system weight (approximately $40 \mathrm{grams}$ ), that led to the novel and efficient internal thermal design of the $P G$ insulators. This concept was promoted by both APL and the Fairchild Corporation during the latter development stages of the LWRHU (circa: early $80 \cdot \mathrm{s}$ ). 


\section{REENTRY CONDITIONS}

The mission profile for Galileo includes launch by the space shuttle into low earth orbit and subsequent boost into the Venus Earth Earth Gravity Assist (VEEGA) trajectory. The VEEGA trajectory involves two passes through the earth's gravity field, both of which have some small probability of accidental reentry. This mission profile results in a broad spectrum of possible reentry scenarios, ranging in severity from orbital decay to the very high speed VEEGA reentries. Detailed analyses of typical reentry scenarios, other than VEEGA, were provided in Reference 2. The new, more severe, VEEGA trajectory is the subject of most of the attention in this report.

The Galileo/VEEGA earth reentry breakup analysis conducted by the Jet Propulsion Laboratory (JPL), Reference 3, provided the basis for the initial velocity and altitude conditions selected for the LWHRU analysis. Subsequent detail on altitude-gamma reentry combinations was provided by A. McRonald, Reference 4, and used by D. Conn to generate the altitude-gamma-velocity charts (Figure 5, Reference 5) used by APL in the reentry analyses.

More specifically, APL chose to examine the expected initial velocity conditions (46,750 fps, inertial frame) and the midrange altitude versus flight path angle variation corresponding to this velocity as shown in Figure 5. The first survey of the VEEGA reentry map in addressing the LWRHU reentry performance was restricted to initial flight path angles of $-90,-50$ and -10 degrees (Reference 1). The $-90^{\circ}$ case would provide representative thermal response behavior for steep reentries to support thermal stress evaluations. The $-10^{\circ}$ case was intended to provide representative thermal and ablation response behavior for shallow reentries where, as indicated later in section VI, thermal and ablation failure events are more likely to occur. The -50 degree condition provides response behavior to moderate flight path reentry environments.

The reentry orientations selected for these analyses are side-on and end-on. The rationale and necessity for these selections are discussed in Section VI. 
IV. GENERAL DESCRIPTION OF ANALYSES

The purpose of the LWRHU reentry analyses is to assess the survivability of the LWRHU under orbital, super-orbital, and VEEGA reentry modes. Computer simulations are used to calculate 1-D ablation, multi-dimensional thermal response, and thermal stress response on the LWRHU and sequential configurations.

The orbital and super-orbital (escape velocity conditions) reentry modes were addressed in the 1985 SAR, Reference 6. For the present analyses, the VEEGA entry modes are emphasized. A discussion of the other scenarios is given in Section $X$.

The reentry trajectory of the LWRHU was simulated at JHU/APL using a three degree of freedom (3DOF) simulation. This 3DOF program was used to generate, in addition to trajectory parameters, the heating rate, $\dot{q}$, stagnation pressure, $P_{t}$, and stagnation enthalpy, $h_{t}$, that are used as inputs to 1-D and multi-dimensional thermal analysis programs. The 1-D CMA program generates preliminary thermal and ablation (recession) response information while the multi-dimensional SHTP programs generate detailed recession and temperature distributions. The distributions are used as inputs to a program that is used to evaluate the structural integrity of the module due to thermal stress - the SAAS program. These analytical computer programs are more fully described in the pertinent sections on thermal and stress analyses. 


\section{AERODYNAMICS}

The LWRHU and LWRHU component aerodynamics used as inputs to the 3DOF trajectory simulation consist of the drag coefficient,

$$
C_{D}=\frac{d r a g(1 \mathrm{bs})}{1 / 2 \rho V^{2} S}
$$

where

$$
\begin{aligned}
& \rho \quad=\text { density in slugs/ft } \\
& \mathrm{v}=\text { velocity in } \mathrm{ft} / \mathrm{sec} \\
& \mathrm{S}=\text { the reference area in } \mathrm{ft}^{2} .
\end{aligned}
$$

These are given as either a function of Mach number and altitude or as a function of Mach number in the continuum flow regime and a bridging function for interpolating between continuum and free molecule flow. A constant value of angle of attack is assumed. Side-on and end-on orientations have been selected for LWRHU thermal and thermal stress analysis. The aerodynamic estimates used as inputs to the 3DOF simulation, Reference 7 , are given in Table 1 for these orientations. Since aerodynamic data for cylinders of these low fineness ratios $(=1.2)$ are very sparse (especially in the free molecule regime), these aerodynamic estimates are based on a variety of data found on blunt bodies - disks, plates and some cylinders.

The drag coefficient at terminal velocity conditions of the LWRHU, and the terminal velocity were estimated, Reference 8 , in support of impact tests performed by Los Alamos National Laboratory, Table 2. The cross-flow drag (and consequently terminal velocity) is highly dependent on the wall temperature. The transition from cold wall drag to hot wall drag is dependent on Reynolds' number and surface roughness. The expected ranges in these parameters for the LWRHU are such that either cold or hot values are possible, Table 2.

The drag coefficients for the side-on orientation of the LWRHU clad/fuel assembly and for the fuel pellet were assumed to be the same as for the LWRHU, except that the reference areas are appropriate for these components viz: $0.001074 \mathrm{ft}^{2}$ for the clad/fuel assembly and $0.000583 \mathrm{ft}^{2}$ for the fuel pellet. The estimated terminal velocities at sea level are $162 \mathrm{ft} / \mathrm{sec}$ for the clad assembly and $119 \mathrm{ft} / \mathrm{sec}$ for the fuel pellet. For the end-on orientations the terminal velocities are about the same as for the side-on orientation. 
THE JOHNS HOPKINS UNIVEASITY APPLIED PHYSICS LABORATORY LAURel maryland

VI. THERMAL ANALYSIS

A. Discussion

1. Reentry Design Evaluation Versus Design Development

Although the effectiveness of the LWRHU design has generally been demonstrated for reentry environments resulting from direct orbit accident scenarios, the reentry design boundaries clearly do not extend to the high energy reentry environments associated with the Galileo/VEEGA maneuver. For a VEEGA reentry safety assessment, the LWHRU is positioned fully into a design evaluation process as opposed to the earlier design development process implemented for the direct orbit scenarios. Although both processes require scrutiny of the design's thermal, ablation and structural failure modes, the scope of the processes are clearly different. In the design development mode, concentration can be narrowed down to particular reentry design corridors as indicated in Figure 6 . However, because of the severity of environments with the VEEGA situation, the issue is not whether failures might occur but one of determining the extent of failure zones. Consequently, the evaluation process shaped by VEEGA must consider an enlarged $V-\gamma$ map as the domain of interest and greatly increases the scope of the reentry safety analysis.

It was assumed for this study that VEEGA initiated entries, as defined by initial flight path angles, will range from the escape boundary at the shallow end to the maximum -90 degree case. All reentries within this maximum range were assumed to occur with equal and likely probability since a VEEGA probabilistic reentry map was not available in time to direct this study into those zones of higher probabilities. The total probability for VEEGA reentries, as estimated by JPL, is less than $10^{-6}$ per mission.

\section{VEEGA Reentry Safety Evaluation Process - Guidelines}

Ground rules were established on a prioritized basis to keep the VEEGA reentry safety evaluation process within manageable limits. These ground rules are listed in Table 3 for the network of VEEGA reentry analyses. Although aeroshell temperatures are generated in support of thermal stress evaluations, it is emphasized that the thrust of section VI is to address the ablation and thermal response performance of the LWRHU for the VEEGA environments. The structural response is addressed in section VII.

\section{B. Assumptions}

1. Initial Reentry Conditions

The initial primary reentry conditions for all LWRHU assemblies are as given in section III. These primary reentry evaluation cases were supplemented in the thermal analyses by evaluations at -4.5 and -30 degrees to further define LWHRU response behavior over the entire VEEGA $V-\gamma$ map. 


\section{Reentry Configurations}

This study considered a simplified three stage sequential breakup scenario consisting of (a) a full LWRHU assembly followed by (b) an instantaneous breakup and release of the clad fuel assembly followed finally by (c) instantaneous breakup and release of an integral fuel pellet should failure events occur during the course of reentry. Neither fractured segments of the pellet nor particle fines, possibly produced during assembly or the prior phases of the mission, have been considered as discrete reentry bodies upon a clad failure event.

\section{Reentry Orientation}

This category, perhaps more than any other, serves to illustrate just how rapidly a reentry safety evaluation can propagate in size and why restrictions become necessary. Each of the reentry configurations discussed above can, from a idealistic, analytically expedient viewpoint, assume three orientations: side-on stable, end-on stable and tumbling. In truth, reentry orientation is a dynamic, stochastic process dependent upon applied forces and moments resulting from structural breakup and release. Consequently, given a reentry environment that leads to a complete breakup sequence, and that further includes all orientation permutations, 27 cases (from solely an orientation perspective) would be required to analyze a single reentry condition. Multiply this potential requirement by four or five initial reentry conditions as considered in this study and one comes to understand why only a limited number of scenarios may be examined.

The primary orientation assumed for the LWRHU assembly and subsequent breakup configurations was a sustained side-on stable attitude from initial reentry through to impact. A preferred (or stable) reentry orientation is a hotter condition than a dynamic, tumbling mode and, of the two idealistic stable attitudes, previous design studies indicate a side-on orientation will more likely produce a clad melt event. Of all the in-flight failure modes, the clad melt event bears the greatest significance since it nearly always results in fuel release (if not during flight, then ultimately at impact).

Limited cases of LWRHU reentries for an idealized end-on stable orientation were also examined as discussed in section VI. F. Clad/fuel assembly and fuel pellet reentry analyses need to be conducted to examine the effect of orientation on melting.

4. Failure Criteria for Ablation and Thermal Response

The threshold failure criteria used in this study to identify ablation and clad melt failure events are an attempt to recognize the uncertainties that affect analyses for high energy reentries such as the VEEGA family. These criteria are subjective, relying on engineering intuition rather than hard statistical analyses. In most cases, statistics are simply 
not available for the numerous environmental and response variables that determine these failure modes. A sensitivity analysis needs to be conducted to provide the effect of uncertainties on the LWRHU reentry response parameters and related failure conditions.

Two criteria are specified for the melt failure. The threshold failure temperature for those members protected by insulation is the platinumcarbon eutectic temperature minus $300^{\circ} \mathrm{F}$. This corresponds to $\left(3660-300^{\circ}\right)=$ $3360^{\circ} \mathrm{R}$ for the LWRHU's Pt30Rh member. For non-insulated members, the threshold is 1 owered by $500^{\circ} \mathrm{F}$ (or $3160^{\circ} \mathrm{R}$ for the Pt30Rh clad) since there will now be greater sensitivity via direct exposure to environmental uncertainties.

One final thought regarding the uncertainty band formed by the failure threshold (e.8., the 300 or $500^{\circ} \mathrm{F}$ temperature band on melt failure) relates to probability of occurrence. The farther a response variable such as 'aeroshell recession' or 'clad temperature' is predicted to penetrate this uncertainty band, the greater the probability of occurrence for this failure event. Here again, probabilities are definable only in a qualitative sense (high, low, etc).

\section{Breakup Criteria}

This category is closely related to the previously discussed failure mode criteria but requires separate identity and specification to define the sequential breakup analyses. In this study, the failure thresholds for both the ablation and melt failure modes at the side-on stagnation location were chosen to identify an instantaneous catastropic failure for either the LWRHU or clad/fuel assemblies with resulting instantaneous release of its contents. Fuel pellet melting is also initiated at the threshold of the $500^{\circ} \mathrm{F}$ uncertainty band for all locations on the pellet. However, the issue for bare fuel reentry is not one of failure identification but rather determination of the pellets's melt mass fraction during entry.

\section{Methodology: Side-on Analyses}

\section{Zonal Approach for VEEGA Reentry Safety Evaluation}

A zonal approach over the VEEGA reentry $V-\gamma$ map was selected to represent reentry performance. This zonal evaluation strategy is illustrated by Figure 7 which shows five discrete zones ranging from Zone A for shallow reentries (that are critical to both the aeroshell ablation and clad melt failure modes) to Zone $E$ for steep reentries (which are critical to aeroshell thermal stresses).

The strategy was formulated for the side-on analysis but the general approach is applicable to any orientation.

The boundaries of the zones are given by the dashed lines with the overall map defined by the escape condition as the left extremum and the 
vertical entry as the right extremum. Within each zone exists a solid line that represents a reentry evaluation condition for the side-on LWRHU assembly. Those conditions labeled with a ' $P$ ' represent the primary evaluation sites selected for this study while those labeled ' $S$ ' were supplemental conditions subsequently evaluated to provide a more definitive response description over the entire reentry map.

To further illustrate, the reentry evaluation condition for zone $E$ is the vertical ( -90 degree) flight path angle which also happens to be the right hand extremum for the VEEGA reentry map. The response behavior of a side-on LWRHU determined for this reentry condition is taken to be representative for the entire zone ( $1 . e .$, from -70 to $-90^{\circ}$ ). Likewise, the LWRHU response predicted for the $-50^{\circ}$ reentry is representative of Zone $D$ (from -40 to $-70^{\circ}$ ) and so on.

This zonal map indicates that resolution on response behavior increases with decreasing flight path angle. In Zone $E$, this resolution is a relatively coarse $20^{\circ}$ on flight path angle since environmental sensitivity to initial flight path angle is low in this region of the map. The resolution has sharpened to $10^{\circ}$ in Zone $C$ and becomes even $f$ iner in $A$ and $B$ where environmental sensitivities to flight path angle are large.

Figure 8 shows a closeup of the region at the left hand extremum of the VEEGA reentry map. For the side-on orientation, three degree of freedom (3DOF) trajectory analyses indicated the LWRHU would encounter an initial pass into the atmosphere and subsequently exit in an escape trajectory for initial flight path angles of $-4.0^{\circ}$ or less. By increasing the flight path to $-4.25^{\circ}$, the LWRHU's assembly is captured on the second pass by the earth's gravity force field and will reenter either to the extent of structural breakup or, in absence of failures, all the way to impact. A further increase to $-4.50^{\circ}$ resulted in capture of the LWRHU on its initial pass and demonstrates the sensitivity to flight path angle in defining, for the side-on LWRHU, the various classes of reentries. It is noted that the $-4.50^{\circ}$ condition was also selected as a supplemental reentry thermal evaluation site in this study. As indicated in Figure 8 , the escape boundary $\left(-4.125^{\circ}\right)$ and the multiple pass boundary $\left(-4.375^{\circ}\right)$ were taken to be the mid-values based on the cases examined in the trajectory analyses.

\section{Reentry Trajectory Analyses - Side-On Stable LWRHU}

Since idealized side-on stable orientations were assumed for the LWRHU assembly as well as for subsequent breakup configurations throughout reentry, trajectory analyses were restricted to three degree of freedom flight dynamics as opposed to more complicated 6DOF simulations. The 3DOF code used in these analyses has been documented in Reference 10.

Table 4 provides the initial conditions for the side-on LWRHU corresponding to zones A through $E$ defined for the VEEGA reentry map. As previously discussed in section III, these initial conditions were obtained 
THE JOHNS HOPKINS UNIVERSITY

APPLIED PHYSICS LABORATORY

LAUAEL MARYLAND

from the JPL breakup study conducted for the Galileo spacecraft to identify GPHS module release events. This table also states other conditions specified for the analyses. It is emphasized that the trajectory analyses do not contain ablation coupling effects in terms of either changing weight (i.e., mass loss) or changing aerodynamics (via shape change) as a function of reentry flight time. This omission is due to limitations in the available trajectory and heat transfer computational codes at APL. For side-on reentries, it is believed that ablation coupling would result in a lighter and more blunt flight configuration, thereby reducing the LWRHU's ballistic coefficient. This has a benign effect in reducing impact environments (i.e., clad temperature and velocity) should the LWRHU assembly survive the reentry's heat pulse. Additionally, a lower ballistic coefficient will also act to reduce the thermal environment; however, this effect will be counteracted by increased local convective heating rates due to geometry scaling and shape change influences. The net effect to these opposing behaviors represents one of the larger uncertainties in the study.

The drag coefficients, reference area, and weights corresponding to the three possible flight configurations, and used in the 3DOF trajectory analysis, are given in section $V$.

Figure 9 shows the representative trajectory profiles over the hypersonic heat pulse period for each of the zones of the Galileo-VEEGA reentry map. There is a remarkable insensitivity of the trajectory track and hence reentry environment to flight path angle in Zones $C$ through $E$. This close similarity suggests that a failure mode occurrence in one zone (e.g., aeroshell thermal stress) will likely occur in all three zones and represents a sizeable region of the total reentry map. Flight path sensitivity becomes more evident in travelling from zone $C$ to Zone $B$. The totally different character of the Zone A profile is typical of minimum gamma trajectories for prompt reentries that show an intermediate peak in altitude prior to final descent to impact. These type of reentries result in a prolonged convective heat pulse and therefore represent severe environments for evaluating the ablation and thermal response failure modes as will be indicated in the next section. The critical or design minimum gamma would be one in which the track peaks at or close to 400,000 feet (i.e., the edge of the sensible atmosphere) prior to final descent.

The heat pulse flight period for all zones terminate at an altitude of about 100,000 feet or greater. This suggests the strong likelihood of high altitude source terms should failure events occur that lead to atmospheric fuel release. It is interesting to note that the location of the maximum convective heating rate for the various zones covers a wide range on altitude (approximately $150 \mathrm{~K}$ to $250 \mathrm{~K}$ ) in contrast to a narrow band on velocity ( $38 \mathrm{~K}$ to $40 \mathrm{~K}$ fps). 


\section{Engineering Methods and Correlations}

Due to the high energies of the VEEGA reentries, the aerothermodynamics for the LWRHU are complicated by the addition of shock layer radiation as a mode of heat transfer. Furthermore, both the radiative and convective modes are influenced by chemistry effects that further complicate the reentry environments. This is illustrated in Figure 10 which shows thresholds for both the dissociation and ionization processes as well as the kinetic threshold as overlays on the trajectory tracks for the various zones of the Galileo-VEEGA reentry map. These thresholds are very approximate but serve to illustrate the various aerothermodynamic regimes encountered by a side-on LWRHU reentry. Also, it is noted that the kinetic threshold shown here is for a one-foot radius-sphere; however, for the smaller LWRHU leading edge, this threshold will be translated to lower altitudes since, for this geometry, the forward (stagnation) shock layer thickness is considerably smaller relative to required kinetic relaxation distances. This figure indicates that the high energy VEEGA reentries result in a highly ionized (and therefore radiating) shock layer in the stagnation region. It also indicates that nonequilibrium chemistry is probably a consideration in the maximum heating region for most of the reentry zones. This has implications regarding both the radiative and convective heat transfer modes as will be discussed later.

The engineering methods used to define the aerothermodynamics for the Galileo-VEEGA reentry analyses are provided in Table 5. The table also gives references providing theory, experimental data sources, code user's manuals and other background information.

\section{a. Shock Layer Radiation}

Engineering charts developed from the RAD/EQUIL code as a function of velocity, altitude and spherical nose radii (via NASA-Langley Research (enter) were used to predict stagnation heating rate histories for the various VEEGA reentry zones. RAD/EQUIL is considered to be a good engineering radiation transport code for stagnation flow and recently demonstrated good performance in predicting ground and flight radiation test data for air (Sutton, Reference 14). The equivalent spherical radius for the side-on LWRHU configuration was based on equating the LWRHU's stagnation shock layer thickness (i.e., shock standof $f$ for adiabatic flow) with the stagnation shock standoff for a spherical geometry with nose radius $R$ (i.e.,

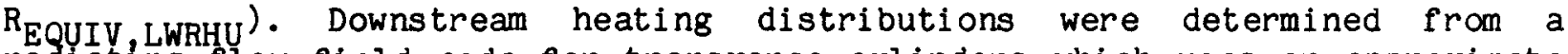
radiating flow field code for transverse cylinders which uses an approximate radiation transport model. This code, obtained from NASA-Langley Research Center (Olstad, Reference 15) is felt to provide a good engineering approximation for downstream heating histories in the form of the ratio: $\dot{q}(10 c a l) / \dot{q}(s t a g)$. These ratios are subsequently reduced using the stagnation values obtained from RAD/EQUIL. 
Carbon ablation products can act as an absorption barrier in blocking shock layer radiation to a body's surface. This effect is shown in Figure $11 . a$ and indicates that radiation blockage can be as much as $40 \%$ for high carbon ablation rates. Based on preliminary 1-D ablation analyses for the side-on LWRHU, radiative blockage is shown to reach these levels during the maximum heating region for the steep VEEGA reentry zones. Although this ablation effect is recognized to be important, time constraints on this study did not permit incorporation of radiation blockage into the reentry thermal codes for Galileo-VEEGA.

\section{b. Convective Heating}

Reentry convective heating is comprised principally of a molecular conduction mode (as usually identified with subsonic and supersonic flow) and a mass diffusion mode that results from dissociation and ionization processes occurring in the heated air shock layer. Although this study's stagnation theory (i.e., Detra, Kemp and Riddell, Reference 18) is theoretically founded on a dissociated shock layer condition, its selection is based on its excellent correlation of shock tube experimental data (Perini, Reference 21) that extends into the ionization regime. The comparison of theory with data is shown in Figure 12 and is demonstrated in Reference 21 to be very close to the data's least square fit and superior to various ionization stagnation theories.

Downstream laminar heating for the LWRHU utilized Lee's Mach 3.0 distribution during hypersonic flight and the Mach 2.0 distribution for supersonic flight (based on continuum flow). The distributions are compared with experimental data in Figure 13. The choice of the Mach 2 distribution is somewhat arbitrary; however, this flight period provides less than $1 \%$ of the total heat load. For stabilized reentries, the precision of base heating distributions as shown in Figure 14 will have little bearing in evaluating the ablation or thermal response failure modes due to their relatively low levels. Subsonic cooling distributions for the LWRHU, are based on a compilation of cylindrical heating rates for subcritical flow (Perini, Reference 32). A smoothed version of data is shown in Figure 15 and shows a singular distribution over the windward surface but a family of distributions as a function of Reynolds' number in the subsonic laminar wake. These data were programmed into the subsonic flight analyses to address the impact failure mode.

The effects of low density flow and mass transfer effects on convective heating were accounted for in the study. The blockage effect on convective heating is generally served by the Putz and Bartlett correlation which was developed from a multi-component, reacting boundary layer study for ablating graphite (Putz and Bartlett, Reference 33). However, this correlation did not permit stable solutions for the steep reentry zones, thereby resulting in the substitution of the Mickley-Spalding 'blowing' correlation for this mass transfer effect. As indicated in Figure 11.b, the Mickley-Spalding correlation results in less blockage to convective heating than the more accurate Putz and Bartlett. 
Stagnation heating rates that are required directly by the thermal response models (as well as for reducing distribution rates to obtain downstream heating) are calculated by the trajectory code for a one-foot radius sphere reference geometry. These heating rates are converted to cylindrical stagnation rates by adjusting for (1) flow dimensionality, (2) stagnation velocity gradients due to geometry and (3) geometry scale effects (nose radii). The ratio of stagnation velocity gradients of sphere and cylinder geometries having identical nose radii is shown in Figure 16 . This ratio indicates that a departure from unity is approximately the supersonic/subsonic flight period. Consequently, only the impact failure mode is addressed by this adjustment on velocity gradient (as it affects stagnation convective cooling rates).

\section{c. Applicability of Methods and Correlations}

The methods identified in Table 5 were used for all sequential reentry configurations that maintain a side-on stable flight attitude as considered in this segment of the VEEGA assessment. Except for distribution functions for heating and static pressure, the methods are applicable for endon stable entries as well. The distribution functions for the end-on reentry analyses are discussed in section VI.F of this report.

\section{Summary of Zonal Environments - Side-on LWRHU Reentry Conf iguration}

The 3DOF trajectory analyses provide aerodynamic and aerothermodynamic histories in addition to the basic position, velocity and acceleration data. Table 6 provides for each of the five zones in the study a list of reentry parameters that provide a measure of the Galileo-VEEGA environments for side-on LWRHU flight conditions.

Table 6 displays the typical environmental trends of increasing heating rates, (item 1) decreasing heat pulse periods (item 4) and decreasing time integrated heat loads (items 2,3 ,) with increasing flight path angle (1.e., from Zone A to Zone $E$ ). The high energy VEEGA reentries invoke an additional heating mode (shock layer radiation) in establishing thermal environments. However, the LWRHU reentry thermal environment remains convectively dominant. This is particularly evident in the shallow reentry zones where ablation and thermal response concerns are in the foreground. Acknowledgement of the secondary role played by the radiation mode is important because this secondary effect reduces the degree, or influence, of uncertainties associated with basic radiation transport modelling and radiation coupling on convective heating. The omission of the radiation blockage effect (due to ablation products) in this study has little bearing on the reentry safety evaluation except perhaps for very abbreviated flight periods in the steeper reentry zones. 
As Mach 5 is essentially the terminus for the hypersonic heat pulse period, it is shown by comparison from this table that the heat load (item 3 ) is essentially equivalent to the total heat load (item 2). Therefore, there is a strong argument for stating that failure events leading to atmospheric release of fuel will likely occur at or before the Mach 5 condition. The corresponding altitudes at Mach 5 would indicate that such fuel releases leading to source terms are at sufficiently high altitudes ( $\geq 90000$ feet) to be subject to global dispersion. Exceptions to this argument are the scenarios where (1) prolonged subsonic oxidation induces a heat shield ablation failure and releases a previously melted clad with liberated fuel fragments or (2) a fuel pellet experiences a high altitude release and either partially or fully survives reentry except for fuel particles that may be aerodynamically scrubbed from the pellet's surface. These events could possibly lead to lower altitude source terms.

Other parameters generated by the 3DOF trajectory analyses for the side-on configuration are pertinent to structural environment during flight, viz: maximum stagnation pressure (1.e., aerodynamic loading) and maximum deceleration (inertia loading) conditions (Table 6, items 5,6). Whereas stagnation pressures are moderate ( $\$ 4$ atmospheres) over the entire reentry map, the decelerations have a wide range over the various zones. Future refinements on aeroshell thermal stress evaluations will include inertia loads for the steep reentry zones.

A rough indication of the impact environment is given by impact velocity (item 7) and subsonic flight periods (item 4) for the various zones. For the full LWRHU assembly, all zones essentially acquire terminal (or equilibrium) velocity of about $125 \mathrm{fps}$ at impact (see section $V$ for more detailed analyses on terminal velocity). A long subsonic flight period, which constitutes over $96 \%$ of the total flight time for the steeper zones, suggests an extended cooling period to lower the clad's temperatures without incurrence of brittleness, improve its strength and thereby improve its impact performance.

\section{Thermal Models}

1. Thermal and Ablation Response Considerations

In determining the LWRHU assembly's thermal and ablation response, there are two basic modelling requirements to be considered in addressing reentry environments. The first requirement is one of modelling the LWRHU's structural configuration in variables appropriate for solving the general thermal diffusion partial differential equation. The product of this effort is generally referred to as a thermal model or network since the thermal variables possess electrical analogies. The second modelling requirement is one of simulating the ablation processes operating at the LWHRU aeroshell's external surface. The ablation modelling is obviously coupled directly to the thermal environment as well as to the structural thermal network. These modelling requirements apply to the sequential breakup configurations as well. 


\section{a. LWRHU Thermal Models}

Solutions to the thermal diffusion equation must be done numerically since, for reentry problems such as the VEEGA scenarios, the equation itself is highly nonlinear and the required boundary conditions are nonlinear in both time and space. A numerical approach requires subdividing the various structural components into discrete elements or nodes with each of their center of mass being a spatial location for a calculated temperature history as the solution marches in time along the reentry trajectory. Each node is characterized by a thermal capacitance and thermal conductance (connecting adjacent nodes) wherein the total composite is termed the thermal network. The two-dimensional (2-D) thermal network for the LWRHU assembly considered in the side-on analyses is shown in Figure $17 . a$ and represents a cross-sectional view in cylindrical coordinates $(R, \theta)$ taken at the mid-plane of the assembly. The network is described by a $12 \mathrm{R} \times 7 \theta$ notation for a total nodal count of 84. The second thermal model shown in this figure is the side-on clad/fuel assembly reentry configuration (Figure 17.b) that would result from either an ablation or thermal stress failure of the LWRHU aeroshell. This model was lifted directly from the assembly model in Figure 17.a. Finally, the third model considered was the 2-D side-on fuel pellet configuration (Figure 17.c) that would result from a clad melt failure of the Figure 17.b configuration. This model shows an enhanced spatial grid fineness for the pellet from that shown in Figure 17.a to improve on the resolutions of melt mass fraction during reentry.

\section{(1) Interface Heat Transfer}

The modes of heat transfer that are typically considered at an internal interface are thermal radiation, gaseous conduction and solid conduction. The first two will be operative for interfaces that are 'open' (i.e., a gap between components) and the latter for 'closed' interfaces (components in intimate contact). In some instances (e.g., high altitude flight), only radiation exchange will be considered as a heat transfer mode. Forced or natural convection models historically have not been considered as an additional heat transfer mechanism in reeentry safety analyses.

Prior reentry design studies for parking orbit related scenarios considered vacuum gaps that eventually became filled with helium due to helium release from the plutonia fuel. Release was somewhat arbitrarily related to threshold temperature levels specified for the clad member (Appendix G, Reference 43). A more sophisticated model for the fuel's helium release along with considerations of gas infiltration and extraction through the LWRHU aeroshell's permeable wall provided a clad thermal response that was closely approximated by use of a one atmosphere air fill in the gaps from initial reentry to impact (Appendix $G$, Reference 44). The simpler air fill model was chosen for the VEEGA side-on thermal models to expedite the study. Gaseous conduction is calculated from the fundamental Fourier heat conduction law. Thermal radiation across the interface gaps considers a radiation 
exchange factor based on parallel plate geometry and limits radiation to just the opposing set of nodes due to the small sizes of the gaps. The gap sizes are shown in Figure 17.a and have been assumed to be uniform circumferentially and constant with time.

\section{(2) Boundary Conditions}

The numerical solution of the diffusion equation requires an initial value of the LWRHU's temperature distribution and two boundary conditions for each spatial independent variable. The initial temperature distribution is generally taken to be the steady state operating temperature in space. Steady state distributions will likely differ for each of the numerous units aboard Galileo since such distributions are a strong function of the packaging configuration. As an expedient, this study has borrowed from the earlier LWRHU design studies by assuming that all the Galileo units possess the same initial steady state temperature based on direct exposure to space. This distribution is provided in Table 7 .

One of the specific boundary conditions for the space variables that is common practice in all thermal modelling exercises is the use of adiabatic (or insulated) surfaces that results from the intrinsic symmetry of the problem. This use of symmetry is displayed in Figure 17.a by considering only one-half the 2-D cross section for the side-on reentry orientation.

The other boundary conditions are tied to the energy balances at all internal and external surfaces for the LWRHU's various components. For the configuration shown in Figure 17.a, all internal surfaces have the following boundary conditions specifications throughout the solution's march in time for a reentry:

$$
\begin{gathered}
\text { Solid heat conduction }\left(\begin{array}{l}
\text { to surface } \\
\text { from }
\end{array}\right) \text { equals } \\
\text { gaseous heat conduction plus radiation }\left(\begin{array}{l}
\text { from surface } \\
\text { to }
\end{array}\right) .
\end{gathered}
$$

The external (or aerodynamic) surfaces' energy balance is the major requisite of the reentry solution by coupling the reentry environment to the structural response of the reentry body. The general specification of an energy balance for a reentry problem with surface ablation processes is defined by Figure 18. This boundary condition is applicable for the LWRHUVEEGA reentries. The energy term coupling the environment to the structural response is the solid conduction term, $\dot{q}$ (cond).

\section{(3) Energy Source Term}

One additional consideration to be included in the solution of the diffusion equation is the energy source term for the plutonium fuel. For this study, it is assumed that the fuel's one thermal watt of energy is uniformly distributed over the entire pellet. Impact is the only failure mode 
in which this term may be influential by providing a contribution in governing clad impact temperatures.

\section{b. Ablation Models - Carbon-Carbon Material}

Figure 19 shows the surface mass balance that is generally considered in developing an overall scheme for ablation modelling of carboncarbon materials. For this study, mass loss due to mechanical ablation (e.g., aerodynamic shear loads) was not considered and represents a major uncertainty in addressing the LWRHU's ablation failure mode. This uncertainty, along with other uncertainties in modelling discussed in various sections of this report, constitute the rationale for the use of a $50 \%$ wall thickness failure criterion. Arc jet ablation tests for the LWRHU that are in progress (see Section VIII) may provide insight for evaluating this ablation term; however, test results were not available in time to influence this study. An approximate mechanical erosion model has been developed for ATJ graphite (Reference 45) based on the experimental data of Lundell and Dickey (Reference 46). However, the processing and construction of the LWRHU's 3-D carboncarbon aeroshell was felt to be too disparate from the bulk graphite morphology to consider using this erosion model.

There are three primary thermochemical ablation processes to be considered for carbon-carbon (or graphite) materials. These are (in the direction of increasing surface temperature): rate limited (kinetic) oxidation; diffusion oxidation; and, sublimation (Figure 20). The thermochemistry in place for this study considered the 'moderate' rate limited mass loss schedule shown in Figure 20 with related reaction energies corresponding to the relationships provided by Hunter (Reference 47) for a glowing combustion process. This process is defined by the reaction:

$$
\mathrm{O}_{2}(\mathrm{~g})+2 \mathrm{C}(\mathrm{s})+2 \mathrm{CO}(\mathrm{g})
$$

The diffusion limited oxidation plateau is also based on carbon monoxide as a product of reaction and represents the limiting solid carbon consumption rate for an oxidation process. As the name implies, this limit is imposed when reaction rates become so fast with increasing temperature that oxygen (oxidizer) consumption becomes controlled by the time it takes oxygen to diffuse across the boundary layer to feed the reaction.

The sublimation model is based on equilibrium thermochemistry for 16 species including the important carbon vapor molecules $C_{1}$ through $C_{5}$ which take on increasingly dominant concentrations as temperatures increase in this regime. This $\mathrm{C}_{1}-\mathrm{C}_{5}$ JANAF model showed good performance in correlating the experimental sublimation mass loss data of Lundell-Dickey (Reference 46). It has been demonstrated that an equilibrium model will result in higher predicted mass loss rates than a more realistic nonequilibrium vaporization model (Reference 48). Further background on the APL ablation models can be obtained from References 45 and 47. 
THE JOHNS HOPKINS UNIVERSITY APPLIED PHYSICS LABORATORY LauRel maAyland

One final note regarding carbon ablation modelling has to do with representation. Mass loss rates determined from these various ablation processes are cast in the form of a mass transfer parameter, $B$, defined as surface mass loss rate divided by a selected mass transfer coefficient. Mass transfer coefficient is a means of representing mass diffusional fluxes at a surface through the use of a mass concentration driving potential in a manner analogous to representing surface heat transfer by use of a heat transfer coefficient and a temperature or enthalpy driving potential. In fact, the usual approach is to relate the mass transfer coefficient to heat transfer coefficient (generally a function of Lewis No.) due to the similarity of the mass and thermal diffusion equations. The preferred approach is to use the mass transfer coefficient correlation of Putz and Bartlett that was developed from multicomponent, reacting (i.e., equilibrium thermochemistry) boundary layer analyses for ablating graphite surfaces (Reference 49). The algorithm for Putz and Bartlett's heat and mass transfer correlations was circumvented when the alternate algorithm containing the Mickley-Spalding mass transfer correlation was invoked to compute the convective heating 'blowing' effect (as previously discussed in section VI.C.). This algorithm sets the mass transfer coefficient equal to the heat transfer coefficient (by assuming $a$ Lewis No. of unity) which will result in conservative mass loss rate predictions for the VEEGA reentries experiencing strong sublimation (i.e.,

$B>0.5)$. The mass transfer coefficient approach for high temperature ablation problems has been canonized through the widespread use of Aerotherm Corporation's CMA and companion EST codes (References 50 and 51). Further background on this approach is provided in the six part series of Reference 52 and in Reference 50.

\section{c. Ablation Models: Clad and Fuel}

For those reentries yielding sequential breakup configurations of, initially, the clad/fuel assembly followed by a bare fuel pellet, the ablation response becomes much more complicated than summarized above for the LWRHU carbon-carbon aeroshell. This complication arises from the introduction of a melt process. From a modelling viewpoint, predicted response behavior must consider a three phase problem consisting (from interior to exterior) of a solid core, a melt front followed by a melt layer and finally, vaporization at the melt layer's external surface. Both the melt and vaporization fronts represent moving boundaries with the velocities being a function of the thermal environment and the material's heats of formation in undergoing phase transformation. Further modelling complications arise as the melt layer grows in thickness due to interaction of aerodynamic loading. Such loading can lead to two very important, coupled events:

(1) the molten shape of the reeentry body will drastically deform with the resulting shape change affecting both the trajectory behavior and resulting reentry environment, and 
(2) the melt layer becomes ultimately subjected to mechanical erosion as well as surface vaporization losses with erosion taking the form of either melt layer stripping due to aerodynamic shear or ballooning (or parachuting) due to normal loads.

The precision of modelling a melt problem is considerably more demanding for bare fuel reentries since the disposition of the melt layer will dictate the radiological source term during flight. For example, with melt layer erosion, droplet size of fuel from the parent melt layer may be either directly respirable or may possibly break up into finer droplets, etc.

As the computational capability to address the reentry melt problem is not available, the melting event for the clad/fuel assembly and the bare fuel configurations could be addressed only in the most simplistic terms. A simple thermal response analyses was conducted for both configurations which included conditions of (1) a chemically inert surface (2) no shape change effects and (3) no mass removal due to vaporization and mechanical erosion. An approximate approach to account for heat of fusion was attempted for bare fuel reentries by lumping this heat into the material's

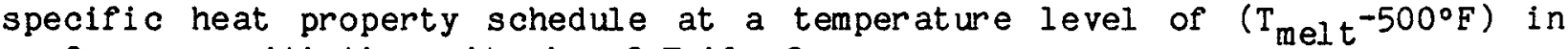
conformance with the criteria of Table 3.

Further perspectives on the fuel reentry problem are available in Ref erences 53 and 54.

\section{d. Material Properties}

Determination of the thermal response of a reentry structure requires specification of the thermodynamic property (specific heat) and the transport property (thermal conductivity) for all materials involved in the design. The need to obtain numerical solutions to the heat diffusion equation is caused, in part, by the problem's nonlinearity resulting from the strong dependence on temperature of the above properties. In addition to these properties, the solution of the surface energy balance (Figure 18) requires information on the thermal radiation properties, absorptivity and emissivity, to determine absorbed shock layer radiation and surface re-radiation. Additionally, these properties are required to determine radiation transfer at the internal interfaces.

The material properties for the various components are given in Table 8.a through 8.f. The properties are the same as used in earlier design studies except for the fuel. The selected fuel properties for the side-on studies are the result of a more recent property survey (Reference 55). The units expressed in Table $8 . a$ hold for all other Table 8 entries.

Due to insufficient information on absorptivity, particularly at the higher temperatures, this property was assumed equal to the more readily avallable hemispherical emissivity for all materials. 
There is mild anisotropy in thermal conductivity for the carboncarbon aeroshell in going from either the $\mathrm{x}$ or $\mathrm{y}$ direction to the $\mathrm{z}$ direction for the orthogonal layup of the material, Reference 56. Since the $z$ direction of the carbon-carbon aligns with the LWRHU's longitudinal axis, and given the assumption that thermal conductivity is essentially equivalent in the $x$ and $y$ directions, the thermal conductivity in the required radial and circumferential directions (via thermal model, Figure 17.a) can be shown by vectorization to be equivalent to the $\mathrm{x}$ (or $\mathrm{y}$ ) direction values ( $\mathrm{Table} 8 . \mathrm{A}$ ).

The anisotropy of the material properties of pyrolytic graphite is significant as indicated by the comparison of the radial thermal conductivity (Table 8.b) to the circumferential value (Table 8.c). In using pyrolytic graphite as an insulator, a designer must be cognizant of the material's high conductivity direction as was demonstrated for the LWRHU design in an earlier discussion.

The latent heat of fusion for the clad alloy, Pt30Rh, was determined by using the heats of the constituent elements (Reference 57) times the respective weight fraction for the alloy. The resulting value is a moderate $57.5 \mathrm{Btu} / \mathrm{lb}$. On the other hand, the latent heat for $\mathrm{PuO}_{2}$ is about twice as high at $112.0 \mathrm{Btu} / \mathrm{lb}$ (Reference 55) and, as previously indicated, was incorporated into the specific heat schedule over a five degree band as shown in Table 8.f.

Gaseous conduction at the interface gaps was based on the thermal conductivity of air (at one atmosphere) using the National Bureau of Standards's schedule for temperatures at or below $2500^{\circ} \mathrm{R}$ and the Yos model for temperatures greater than $2500^{\circ} \mathrm{R}$ (Reference 58 ).

High temperature problems such as reentry are not only difficult to address due to the involved physics but also due to the uncertainty of physical property data required for such evaluations. The thermophysical data of Table 8 is no exception in that the high temperature entries are based on extrapolations, or engineering judgement, from a lower temperature data base and represent a major source of uncertainty in studies of this type. This is particularly true for the fuel reentry problem where the focus is on the behavior of a high temperature melt layer.

\section{e. Numerical Algorithm for Response Solutions}

The numerical solution of the diffusion equation was based on a simple explicit (forward) finite differencing scheme that has been a standard in computational heat transfer for many decades (Reference 59). Minimum computational time steps for stable solutions are based on $90 \%$ of the nodal minimum value of (thermal capacitance/summation of thermal conductances) and ranged from 0.005 to 0.05 seconds for the thermal models of Figure 17 . 
The shortcoming of this algorithm is its inability to shrink the aeroshell's thermal network in response to surface ablation calculations. The network as shown in Figure 17.a, remains fixed during reentry and will have perturbations on the reentry safety evaluations by increasing the aeroshell's thermal stresses, increasing ablation response, increasing clad impact temperatures and decreasing peak clad temperature in addressing the reentry failure modes. The extent of these perturbations are not known at this time but are felt to be small. An algorithm that provides for nodal shrinkage (Reference 60 ) was initially attempted in this study but was abandoned due to repeatedly unsuccessful attempts to execute the steep VEEGA reentries. The other noted shortcoming with this algorithm is the absence of shape change feedback on flight dynamics and aerothermodynamics.

The surface energy balance uses the iterative Newton-Raphson scheme in a manner similar to Aerotherm's CMA code (Reference 50) except the independent variable in this algorithm is the surface temperature. An energy balance is declared when the summation of all the energy terms results in a net energy residual of $1.0 \mathrm{Btu} \mathrm{ft}^{-2} \mathrm{sec}^{-1}$ during hypersonic flight and $0.1 \mathrm{Btu}$ $\mathrm{ft}^{-2} \mathrm{sec}^{-1}$ during supersonic/subsonic flight.

\section{E. Results of Analysis - Side-on Stable LWRHU Response Behavior}

\section{Reentry Configuration: Side-On LWRHU Assembly}

Restricting attention to just the ablation and thermal failure modes, the important response variables become (a) aeroshell stagnation recession, (b) clad maximum stagnation temperature and (c) clad impact temperature. The initial step in this serial evaluation was to conduct reentry thermal analyses for all VEEGA reentry zones to allow comparisons of the response variables against the specified failure criteria. Zones $C$ through $E$ reentries were investigated entirely through to impact whereas, in anticipation of ablation failure events, evaluation for Zones $A$ and $B$ were shortened to just the hypersonic heat pulse (1.e., to Mach 5.0).

The aeroshell's stagnation ablation response for the various zones is given in Figure 21. The response shows a correspondence to total heat load (Table 6) as expected. Recessions greater than $50 \%$ are indicated for Zone A, $B$, and $C$ if the analysis is continued through the heat pulse. The recession values in these Zones, Figure 21, correspond to approximately $100 \%$, 70\%, and $60 \%$ recession, respectively. However, under the terms of ablation criterion of Table 3, aeroshell failure is deemed to occur when $50 \%$ recession occurs which for Zones $A, B$, and $C$ is before the end of the heat pulse. Zones of $D$ and $E$ are just below the failure threshold. The extent that the total recession predictions exceed the $50 \%$ wall failure threshold provides at least a qualitative sense of the probability of encountering an aeroshell ablation failure event. From this perspective, Zone A indicates a very high probability of occurrence based on the specified criteria for this study. The failure probability of occurrence for Zone B, etc., will become progressively lower with increasing flight path angle. 
Figure 22 provides zonal information on maximum clad temperature at the stagnation location and clad impact temperatures. These thermal response data should be interpreted independently of any indications of prior ablation or thermal stress failure events that would lead to a change in reentry configuration. Therefore, for a complete side-on LWRHU assembly reentry configuration, only Zone A decisively indicates the occurrence of a clad melt failure event although Zone $B$ is only marginally below the failure threshold. The clad impact temperatures for zones $C$ to $E$ range from 1160 to $1210^{\circ} \mathrm{R}$ and are well above the ductility transition temperature for the Pt30Rh clad material. Consequently, the only bearing these temperatures $m$ ht have on clad impact performance is possible strength degradation. This consideration should be factored into any review of available LWHRU experimental impact data.

When combining the information provided in Figures 21 and 22 , it is indicated from these reentry analyses that the ablation failure event will precede the clad melt event for prompt reentries in Zone A.

\section{Reentry Configurations: Side-on Stable Clad/Fuel Assembly}

With the ablation failure and structural breakup criteria specified in Table 3, the release conditions for an intact clad/fuel assembly are as given in Table 9. These release conditions offer further insight on LWRHU sequential breakup patterns for the VEEGA environments. The high release velocities for both Zones $A$ and $B$ indicate a considerable reserve of energy remaining for these reentries such that the occurrence of clad melt, fuel release and subsequent fuel melt events are strong possiblities. The occurrence of these events for Zone $A$ is further reinforced by observing that the clad temperature at release is just slightly below the melt failure threshold. On the other hand, the very low release velocity for Zone $C$ indicates that the thermal environment for reentries in this zone has been largely expended and the clad, even at its low release temperature, will experience cool down and survival for the remaining portion of flight.

Using the information in Table 9 as initial reentry conditions, 3DOF trajectory analyses were conducted for Zones A through $C$ followed by reentry thermal analyses for Zones $A$ and $B$. Here again, in conformance with Table 3 guidelines, the side-on stable orientation was given priority. The thermal response results are given in Figure 23. In conformance with the observations discussed earlier on available reentry energy at the clad/fuel assembly release conditions, clad melt failures do occur very shortly after release for Zone A or B. Very little of the available energy in these zones is expended arriving at this state. However, in Zone $C$, the 3DOF trajectory information indicated that any released side-on clad/fuel assemblies will survive reentry and impact at about $160 \mathrm{fps}$. Reentry thermal analyses were not conducted for Zone C; however, considering the long cool down flight period (i.e., 288 seconds) and the fact that the clad is directly exposed to the environment, it is estimated that clad impact tmeperatures will likely be in the range of local ambient to $560^{\circ} \mathrm{R}$ for this zone. 


\section{Bare Fuel Pellet Side-on Reentries}

The fuel pellet release conditions for Zones $A$ and $B$ are governed by the clad melt event at the stagnation region for the clad/fuel assembly reentry configuration (Figure 23). The melt event included the clad's heat of fusion effect. The pellet release conditions are given in Table 10 and indicate very little degradation in the reentry energies (i.e. velocities) from the earlier release conditions for the clad/fuel assembly (Table 9). The high initial velocity for Zone A reentries indicates the fuel pellet will be subjected to a severe environment and likely experience significant melting. Zone $B$ also indicates sufficient residual energy to cause fuel melting.

The fuel reentry analyses was restricted to acquiring a rough approximation for the mass fraction of the pellet that melts for the two VEEGA zones of interest. Using the conditions of Table 10, trajectory and thermal analyses were conducted for side-on pellet reentries. Computational capabilities were not available to fully analyze the fuel reentry problem as discussed earlier in this section (and in Reference 53). A simple thermal response analysis was conducted which approximated the heat of fusion effect but did not account for any shape change effects nor mass loss due to vaporization or mechanical erosion of the melt layer.

Melt predictions for the pellet using this simplified approach indicated $a$ 14\% mass loss in Zone $B$ and $42 \%$ in Zone $A$. The fuel reentry analyses included sensitivity studies in which (a) base heating levels were increased to $10 \%$ stagnation levels and (b) the fuel's emissivity was decreased to 0.8. These changes had no effect on the Zone $B$ baseline results and only $a$ minor influence on the Zone A mass fraction. Time did not permit extension of the sensitivity studies to other variables, such as the fuel's thermal conductivitiy, to provide estimates of uncertainties on melt fractions. However, it is not unreasonable to think that melt fractions could increase to 75\% in Zone $A$ and $42 \%$ in Zone $B$ if one could adequately model shape change effects and mechanical erosion and had better information on melt layer thermal properties. Consequently, this study is quoting a range on melt fraction as shown in Figure 24 to acknowledge these modelling deficiencies. The altitudes quoted in this figure represent the location for complete resolidification and serves as a lower bound. These results indicate radiological source terms resulting from the melt layer will very likely be high altitude terms.

Some percentage of this range on melt fraction (Figure 24) will have been converted to vapor. Here again, the time constraints placed on this study did not permit prediction of vapor mass fraction (e.g., via a KnudsenLangmuir mechanism) nor the effect on melt fraction due to an end-on or .tumbling reentry attitude for the fuel pellet. 


\section{F. End-on Analysis}

\section{3DOF Trajectory and Other Input Parameters}

The LWRHU end-on reentry stagnation heat transfer environment for the Galileo VEEGA trajectory was computed using the 3DOF code (Reference 61). Two initial flight path angles were chosen for analysis - a steep $\left(90^{\circ}\right)$ and a shallow $\left(10^{\circ}\right)$ angle reentry. These cases are the extreme condition under which thermal stress failure and thermal/ablation failures may occur.

The aerodynamic drag coefficients that simulate the end-on reentry orientation in 3DOF are from Reference 62. The initial conditions for each reentry angle are given in Table 11. In each case, the initial velocity is 46,750 fps (inertial). The corresponding initial altitude is based on the variation of altitude versus flight path angle for the expected initial velocities for discrete LWRHU reentries from Reference 63. This is depicted in Figure 5.

The radiative heating rates are interpolated from a table of values in 3DOF which were installed specifically for the LWRHU end-on geometry. Values for this table were computed by NASA (Reference 64) using the RAD/EQUIL radiation heat transfer code (Reference 65). The RAD/EQUIL code provides a refined spectral definition of air's absorption coefficient and represents an upgrade of the previously used Callis method (Reference 66).

The stagnation heating rates for the LWRHU end-on reentries that were computed using the 3DOF code are discussed below. The stagnation convective heating rates were computed in 3DOF for a reference sphere. These values were converted into values for the LWRHU geometry by using the following conversion factor (FC)

$$
\text { (convective heat transfer factor) } F C=2.4 \text {. }
$$

The convective heating conversion factor is taken to be the ratio of the stagnation velocity gradient for a flat faced cylinder and a one foot diameter sphere as developed by L. L. Trimmer. This factor assumes that the free-stream velocity is greater than Mach 5.0 (which happens to be true for most of the heat pulse). For the stagnation radiative heating rates, no adjustment is necessary because the LWRHU geometry is already included in the tables in 3DOF.

Stagnation heating rates at the shallow and steep flight path angles for a Galileo VEEGA reentry are illustrated in Figure 25.

\section{2-D End-on Thermal Analysis}

a. Summary and Conclusions

A thermal/ablation study was performed for the LWRHU in an end-on reentry attitude for reentry angles of $-10^{\circ}$ and $-90^{\circ}$. The JHU/APL SHTP-E 
THE JOHNS HOPKINS UNIVERSITY

APPLIED PHYSICS LABORATORY

LaUREL MagYLAND

Code, Reference 67, was used in the analysis. A shallow reentry with a flight path angle of $10^{\circ}$ was selected for assessing the possibility of clad melt and aeroshell ablation failures due to the high heat loads generated. A steep reentry with flight path angle of $90^{\circ}$ was selected for a thermal stress evaluation because it creates high thermal gradients.

The results of the thermal/ablation analyses for the end-on VEEGA reentry of the LWRHU indicate that the aeroshell and clad will survive reentry in the cases examined. The maximum aeroshell end-face ablation was predicted to be below the $50 \%$ ablation criteria for aeroshell failure for these cases. Extrapolation of these results to the most shallow flight path angles implies that the $50 \%$ ablation crtieria may be exceeded at these angles. The peak clad temperature was predicted to be slightly above $2460^{\circ} \mathrm{R}$, which is substantially below the eutectic temperature of Pt30Rh.

\section{b. Thermal Model}

The thermal/ablation analysis of the LWRHU in the end-on reentry configuration was performed using the SHTPE ablation code (Reference 67). The analytical assumptions are for the most part consistent with those used in the LWRHU side-on analysis. Assumptions that are specific to the end-on geometry are described below. The LWRHU end-on geometry was represented by a twodimensional 762 node thermal network as shown in Figure 26. It is identical to the network that was used for the analysis in Reference 68. The model includes representations of the aeroshell and end cap, all of the internal sleeves, and the cladded fuel pellet. The aeroshell and insulating sleeves are made of Fine Weave Pierced Fabrictm, a product of AVCO systems Division. The clad is made of platinum alloyed with $30 \%$ rhodium material (Pt30Rh). The end cap holes and the crack around the ( $f$ astened) end cap were not represented by this model. The internal contact conductances between adjacent components were specified according to the values in Table 12. These are also from the analysis in Reference 68. The open fuel/clad gaps were assumed to be helium filled and the end gaps in the aeroshell assembly were modeled as vacuums.

The release of helium from the fuel to the gaps in the aeroshell assembly was not included in this study. This assumption tends to increase the temperature levels of the aeroshell and suppress the temperature rise of the clad. However, based on the results from Reference 68, it is expected that helium in the aeroshell gaps will mainly affect the temperature rate of change of the clad and slightly affect the magnitude (i.e., it will slightly increase the peak clad temperature).

The stagnation heating rate history from the 3DOF computer program provided the boundary conditions for the LWRHU model. The radiation heating rates were applied over the entire windward end-face and were assumed to be equal to the stagnation rate. The convective heating rates were also applied over the entire LWRHU by using the distribution in Figure 27, again from Reference 68. They were converted from reference heating rates to LWRHU heating rates using the conversion factor from the section on 3DOF tra- 
THE JOHNS HOPKINS UNIVERSITY APPLIED PHYSICS LABORATORY

lauael mapylano

jectory. The side heating distribution was taken to be the midrange values defined by the bounds of Figure 27 and the leeward end-face heating was taken to be uniformly distributed at $5 \%$ of the stagnation value.

\section{c. Results}

The results of the 2-D thermal/ablation analysis are reported below for the Pt3ORh clad and the LWRHU aeroshell using the Galileo VEEGA reentry environment. The criterion that is being used for clad melt failure is a clad temperature near the eutectic temperature of Pt30Rh $\left(3660-300^{\circ} \mathrm{R}\right)$. The criterion for ablation failures is $50 \%$ ablation through the aeroshell.

\section{(1) Aeroshell and Clad Temperatures}

The aeroshell clad and temperature histories for the two reentry cases studied are presented in Figures 28 and 29 . The aeroshell temperature drives the clad temperature. The temperature response during the first 29 seconds is shown in Figure 28 for the steep reentry. The observable trend is that the clad temperatures will approach a peak value between 1460$2460^{\circ} \mathrm{R}$ beyond 29 seconds since the aeroshell driving temperature is declining from $2460^{\circ} \mathrm{R}$ at 29 seconds. For the shallow reentry, the temperature response of the first 72 seconds is shown in Figure 29. The trend in this case is that the clad temperatures will peak at approximately $2460^{\circ} \mathrm{R}$. In both cases, the peak clad temperature is expected to be below the eutectic temperature of Pt30Rh by a margin of $500^{\circ}$ to $1000^{\circ} \mathrm{R}$.

\section{(2) Aeroshell Ablation}

The ablation profiles for the steep and shallow reentries are shown in Figures 30 and 31 . Using the $50 \%$ ablation criterion for aeroshell failure, the results of Figures 30 and 31 indicate that the LWRHU aeroshell will probably survive in an end-on orientation for these VEEGA reentry environments. At the aeroshell end cap where the aeroshell thickness is least (0.2018"), the maximum recession is less than forty percent (40\%). This occurs at the perimeter of the end cap for the shallow angle $\left(10^{\circ}\right)$ reentry condition.

\section{(3) Temperatures for Thermal Stress Analysis}

Temperature data were computed for the thermal stress analysis using a model similar to that used for the above analyses. To reiterate, the aeroshell intercomponent gaps were assumed to be vacuum, thus increasing the temperature levels of the aeroshell. All other assumptions were identical except for the following.

The reentry analysis that generates data for the thermal stress analysis was computed using the Putz and Bartlett (Reference 69) 'blowing' correlation with a mechanical erosion model, whereas, the clad and aeroshell thermal/ablation failure analysis used the Mickley-Spalding 
THE JOHNS HOPKINS UNIVERSITY

APPLIED PHYSICS LABORATORY

LAUREL MARYLANO

(References 70 and 71) blowing formulation. Blowing is a mass transfer effect which reduces boundary layer convective heating rates, and mechanical erosion effects are those that remove solid matter from the aeroshell.

The Mickley-Spalding formulation is a general functional relationship for blowing. It can be used to correlate blowing data for an ablating material by the selection of appropriate parameters. It was used in the end-on thermal/ablation analyses to be consistent with the side-on studies.

The Putz and Bartlett correlation is specifically a correlation for graphite mass transfer and its activation in the SHTPE code also results in a crude mechanical erosion model. The mechanical erosion model was installed to compensate for differences in ablation between the sublimation model and experimental investigations of ATJ graphite ablation by Lundell and Dickey (Reference 72). The aeroshell end-face temperatures and thermal gradients calculated for the stress analysis were not signiflcantly different from those obtained using the Mickley-spalding blowing relation at the time of maximum thermal stresses.

\section{G. Comparison of Side-on and End-on Reentry Ablation Response}

The $-10^{\circ}$ and $-90^{\circ}$ end-on reentries that were investigated for thermal, ablation and thermal stress response represent Zones $B$ and $E$ on the Galileo VEEGA reentry map. The predicted maximum recession in terms of percent aeroshell wall thickness is shown in Figure 32 for Zones $B$ and $E$ for both the side-on and end-on reentry orientations. The side-on percentages are based on the maximum recession values presented in Figure 21 and the end-on percentages from the data of Figures 30 and 31 .

Comparison of the recessions for these two zones indicates that the endon attitude produces a milder ablation response and that it will survive across the VEEGA map from Zone $B$ to $E$. For both Zones $B$ and $E$, the end-on recession was about $63 \%$ of the side-on values. Using this proportion in Zone $A$, it is estimated that end-on reentries in this zone will exceed the $50 \%$ wall failure criterion as noted in Figure 32. Furthermore, there appears to be sufficient excess over the $50 \%$ recession level to anticipate fuel release and some melting for the Zone $A$ end-on reentries. Assuming the released fuel pellet reenters side-on, a rough approximation of the melt fraction for Zone $A$ will be the range quoted in Zone B for the side-on analysis (i.e., $14 \%$ to $42 \%$, Figure 24). Further sequential reentry analyses for the end-on study are required to confirm this estimate.

In the shallow angle reentries for the side-on stable orientations another scenario for failure is that in which the aeroshell is assumed not to come apart even through burn-through occurs. For this scenario the following impact configurations may be possible in the reentry zones defined in this study as: Zone A (rentry angle $\left.\gamma \leq\left|7^{\circ}\right|\right)$; Zone $B\left(\left|7^{\circ}\right| \leq \gamma \leq\left|20^{\circ}\right|\right)$; and, Zone C $\left(\left|20^{\circ}\right| \leq r \leq\left|40^{\circ}\right|\right)$. 
THE JOHNS HOPKINS UNIVERSITY APPLIED PHYSICS LABORATORY

LAUREL MAAYLAND

Zone A: Full LWRHU assembly with aeroshell burn-through on windward surfaces leading to direct exposure of underlying PG sleeves, extensive melting and resolidification of clad.

Zone B: Full LWRHU assembly with partial burn-through of aeroshell at the ends with direct exposure of underlying $P G$ insulator end plug, possibly partial clad meltings and resolidification in the near end regions.

Zone C: Full LWRHU assembly with partially ablated aeroshell clad assembly intact.

In assessing the radiological risk, both failure scenarios (ablation leading to release of the fuel pellet an containment of a melted clad) should be considered as possible. 
THE JOHNS HOPKINS UNIVERSITY APPLIED PHYSICS LABORATORY LAUAEL MARYLAND

\section{THERMO-STRUCTURAL ANALYSIS}

\section{A. Summary and Conclusions}

Thermo-structural analyses have been conducted for the LWRHU aeroshell with the new Galileo VEEGA reentry configurations. The previous work of $K$. $R$. Waeber, JHU/APL, during 1979-1982 was reviewed to assess the models and applicability to the current analysis requirements. Side-on stable twodimensional and end-on stable two-dimensional axisymmetric analyses were made for the Galileo VEEGA reentry configurations. These analyses were conducted using the computer code "Stress Analysis of Axisymmetric Solids" (SAAS III) utilizing ablation and thermal information from the SHTP and SHTP-E thermal analyses codes. PDA-PATRAN software on the Apollo computer system was used for pre- and post-processing of the finite element input data and the results.

For the most severe thermo-structural case for the side-on stable configuration, the steep gamma angle low altitude release reentry, the maximum aeroshell temperatures were found to be over $7460^{\circ} \mathrm{R}$. Plastic behavior of the FWPF carbon-carbon material was predicted at these temperatures. A possible compressive failure of the aeroshell due to axial stress early into the heat pulse was also predicted. However, the apparent compressive failure affected only a small area (one-tenth wall thickness) of the outer aeroshell wall near the stagnation point and would not cause a loss of the LWRHU internals. The compressive failure mechanism in the FWPF material is not believed to cause a catastrophic event, as would a tensile failure. At no time during the side-on reentry analysis was a tensile failure predicted in the aeroshell.

For the end-on stable thermo-structural analysis, again in a worst case steep gamma angle low altitude release reentry, no failures were predicted in the LWRHU aeroshell. Maximum temperatures witnessed by the aeroshell were predicted to be about $7460^{\circ} \mathrm{R}$. Compressive and tensile stress safety factors calculated for the end-on stable analysis show survival of the aeroshell through the heat pulse even for conservative linear elastic FWPF material properties. The less severe (from a thermal stress standpoint) medium and shallow angle VEEGA reentry configurations were examined at a cursory level, but as expected, showed little cause for alarm.

Conclusions to be drawn from the results of the side-on stable and the end-on stable thermo-structural analyses are as follows: (1) a catastrophic failure of the LWRHU aeroshell is not expected from the thermal stresses encountered during the predicted VEEGA reentry heat pulses, (2) release of the LWRHU internal components and cladded fuel pellet is not expected to result from the thermal stresses encountered during the predicted VEEGA reentry heat pulses. 


\section{B. Methodology and Assumptions}

Several stress analyses have been conducted (with increasing exactness and complexity) to determine the thermo-structural response of the LWRHU aeroshell to the thermal environment predicted for the Galileo VEEGA inadvertent reentries. Both side-on stable and end-on stable axisymmetric two-dimensional analyses were performed for the Galileo VEEGA reentry configurations. Details of each analysis type are described below. Common assumptions made in both the side-on and end-on thermo-structural analyses are detailed here. Only the aeroshell structure of the LWRHU was considered in the thermo-structural analyses.

The highest thermal heating rates and the largest thermal gradients through the aeroshell wall occur during the steep gamma angle reentry, therefore, this reentry configuration was examined initially. Medium and shallow gamma angle reentries then were examined based upon the results of the steep gamma reentry analysis. Temperature distribution input data were obtained from the finite difference Standard Heat Transfer Program (SHTP) or the embedding version (SHTPE), both capable of multi-dimensional heat transfer calculations. The latter version of the code was developed to analyze ablation heat transfer problems, including the effect of receding material boundaries. Input data for both versions were obtained from the reentry trajectory program, 3DOF.

Thermal stress analyses were conducted utilizing a finite element code called "Stress Analysis of Axisymmetric Solids" (SAAS III). This program is capable of plane strain, plane stress, axisymmetric, stress resultant boundary condition (SRBC) and gap element computations. SAAS III also has the capability of defining linear elastic and bi-linear elastic-plastic temperature dependent material properties.

Previous analyses performed on the LWRHU aeroshell for the launch accident scenario were utilized as a guide in the performance of the VEEGA reentry thermo-structural analyses. Reference 73 is the final report written on these analyses performed in the late 1970's and the early 1980's. The same basic precepts were followed in the VEEGA analyses, but the higher velocities and higher heating rates witnessed in these reentries were expected to result in lower safety margins. Based upon initial comparisons of heating rates, it was believed that fallure of the aeroshell could be possible. The same models for initial geometry models were utilized in the analyses when possible. Figure 33 shows the dimensions which were assumed for the LWRHU aeroshell.

Material properties for the AVCO Fine Weave Pierced Fabric (FWPF) carbon-carbon aeroshell material were also taken from the previous analyses by Waeber. The weave-form of the FWPF consists of stacked layers of woven fabric (in plane) pierced with graphite rods perpendicular to the plane of the fabric (axial). Accordingly, the in-plane orthotropic properties are assumed to be equal, while the axial material properties are different. Very little new material property information has been acquired concerning the FWPF material 
THE JOHNS HOPKINS UNIVERSITY APPLIED PHYSICS LABORATORY

LAUREL mapyland

since Reference 73 was published. Material properties up to $5000^{\circ} \mathrm{R}$ are based upon test data from the Air Force Pan Pilot Production Program. Above $5000^{\circ} \mathrm{R}$, the material properties are based upon bulk graphite properties at $1 \%$ strain. This strain assumption is considered conservative as FWPF is believed to support loads at up to $5 \%$ compressive strain. Further FWPF material property assumptions shall be discussed in the analysis descriptions.

\section{Results of Side-on Stable Analysis}

The steep gamma angle low-altitude release reentry configuration in a side-on stable orientation was examined initially because this was considered the more severe of the two orientations based upon previous similar analyses. Also, the vertical reentry produces the most severe thermal gradients through the aeroshell wall. Initial conditions for this reentry configuration are an inertial velocity of 46,750 feet per second, an inertial flight path (gamma) angle of $-90^{\circ}$ and a free release altitude of 271,000 feet. The approximate thermo-structural response of the aeroshell during the first 6.00 seconds of the reentry heat pulse was determined with the plane stress option of SAAS III. Symmetry in both geometry and thermal loading was assumed. Figure 34 shows the side-on geometry and boundary conditions chosen. A coarse finite element model which consisted of 90 four-noded plane elements was utilized with five elements through the thickness of the aeroshell wall. Figure 35 shows the element and node layout of the coarse model in a rectangular $(R-Z)$ coordinate system. All elastic material properties were used in this initial approximation. Use of these initial assumptions provided a quick and inexpensive method of determining the time of maximum thermally-induced stresses during the reentry.

Temperature distribution data at discrete time steps into the steep gamma angle reentry were obtained from the SHTP code. Because the embedding version of the code experienced instability problems (believed to be directly related to the high heating rates witnessed during this reentry prediction), the initial nonablated structural model was utilized throughout the thermostructural analyses. Figure 36 is a color-coded plot of the nodal temperature distributions as interpolated by SAAS III from the SHTP data. (This photograph was produced in the PDA-PATRAN finite element pre- and postprocessing software on the Apollo computer system.) The time of maximum temperature was found to be 2.40 seconds with a maximum of $7600^{\circ} \mathrm{R}$ on the outer wall surface near the stagnation point. Several interesting observations may be noted from the progression of the thermal gradient with time. Initially, the thermal gradient through the aeroshell wall near the stagnation point was most critical. In fact, as is noted later, the maximum thermally-induced stresses occurred a full second ( 1.40 seconds) before the time of maximum temperature. As the reentry time progressed, the aeroshell increased in overall temperature and the through-wall gradient reduced in magnitude. The thermal gradient along the wall of the aeroshell then became the more important factor, although never as great a concern as the through-wall gradient. As the aeroshell increased in overall temperature, the along-wall thermal gradient progressed further along the aeroshell wall. Another 
important observation drawn from these results, Figure 36, was the reversal (late in the reentry heat pulse) of increasing temperature direction at the stagnation point. Initially, the outer wall was at a higher temperature as expected, but after about 4.40 seconds, the outer wall temperature was lower than that of the inner wall. This led to a reversal of stress direction near the stagnation point at this time, as detailed in the stress analysis results below.

The maximum stresses were found to travel around the aeroshell in a manner similar to the temperatures. Initially, the maximums occurred near the stagnation point, as expected, due to the large thermal gradient through the wall. As reentry time progressed, the highest magnitude stresses began to move along the aeroshell wall. Stress results for each discrete time step of the reentry analysis were compared to determine time of maximum stress. Those evaluated were major and minor principal, hoop, radial and calculated equivalent stresses. Hoop stress was considered to be the most likely mode of failure for the side-on reentry, as in the previous analyses by Waeber (Reference 73). This is a valid assumption as the major and minor principal and equivalent stresses followed the magnitudes of the hoop stress. Radial stress appeared to be of little concern. The largest tensile hoop stress was determined to occur at 1.40 seconds into the reentry heat pulse at the stagnation point inner wall. This is one full second before the time of maximum temperature. The safety factor for this stress was 4.24 (a safety factor less than 1.00 signifies failure). The maximum compressive hoop stress occurred at 1.00 second at the outer wall near the stagnation point. The safety factor for this stress was 3.21. Figure 37 shows a hoop stress time history for the coarse analysis. The progression of the largest magnitude stresses can be seen in this plot, as well as the reversal of stress sign near the stagnation point later into the reentry. An evaluation to determine the minimum safety factors for each stress at each time step was also performed. This was done to locate any elements which, while at lower stress values, may have been more critical than the elements with maximum stresses due to the reduced strengths of these elements at the highly elevated temperatures. The minimum safety factors for both tensile and compressive hoop stresses were found to occur at different times than the maximum magnitude stresses. For tensile hoop stress, the minimum safety factor was 3.67 at 1.60 seconds. For compressive hoop stress, the minimum safety factor was 1.94 at 1.40 seconds. Refer to Table 13 for a listing of both maximum stresses and minimum safety factors for the coarse elastic analysis.

A more refined analysis was performed only for the 1.40 second time based upon the above findings. This analysis consisted of several steps, each more complex. These analysis steps were the same as those used by Waeber in the previous launch reentry analyses in 1981. First, a similar plane stress elastic finite element analysis was run with a finer mesh of 360 four-noded elements with 10 elements through the thickness of the aeroshell wall. Figure 38 shows the layout and element numbering scheme for the fine mesh model. Relative magnitudes of discretization and round-off errors have been identified as a function of element size and computational accuracy. 
Therefore, a finer model should provide more accurate results due to less approximation of the aeroshell geometry in the analysis. The results of this analysis compared well with those of the coarse plane stress analysis. Maximum magnitudes of the tensile hoop stress were lower, while maximum compressive hoop stress at 1.40 seconds increased slightly. This is probably due to better accuracy in the fine model and finer interpolation of the temperature data by SAAS III. Elements near the outer wall surface experienced a slight increase in temperature, while those near the inner wall were slightly reduced. The distribution of stress signs at this time, therefore, account for these magnitude changes. Safety factors witness a similar change in magnitude for this time, as expected.

Next, both the coarse elastic model and the fine elastic model were run with the Stress Resultant Boundary Condition (SRBC) option. The SRBC option of SAAS III is a two-dimensional iterative process which couples in-plane stresses and axial stresses normal to the plane, thereby accounting for axial end conditions of the model (i.e., end forces and moments are equal to zero). It should be noted that this is a highly conservative analysis approach, especially when the assumed physical constraints on the ends of the LWRHU aeroshell do not exist in the actual reentry. Comparison of the results for these two analyses with the plane stress executions show a minor increase in hoop stress magnitudes due to the coupling effect. The maximum axial stresses calculated with the SRBC version of SAAS III were double the magnitude of the maximum hoop stresses. As with the previous comparison between coarse and fine models, the maximum fine model compressive stresses were somewhat higher in magnitude and the maximum fine model tensile stresses were slightly lower. These conservative analysis results also showed minimum safety factors for compressive axial stress which were below 1.00 , thus indicating a possible compressive failure of the aeroshell. (A safety factor of 1.00 indicates a stress equal to the ultimate strength of the material at that temperature). In both the coarse and the fine models, the apparent region of failure is at the outer wall surface from the stagnation point to approximately $45^{\circ}$ around the aeroshell. Table 13 contains a tabulation of the SRBC coarse analysis results. Both the plane stress and the SRBC fine elastic analysis results are tabulated in Table 14.

A further step involved the use of the fine elastic plane stress results to isolate regions of the model which were totally in tension or in compression. If both the in-plane stresses of an element were compressive, then only compressive material properties were assigned to that element. The elastic compressive moduli for FWPF are slightly lower than the tensile moduli. In all the previous runs, only tensile elastic material properties had been utilized, once again giving slightly conservative results. The use of both tensile and compressive material properties results in a more exact representation of the LWRHU aeroshell at the reentry time of this analysis. Only marginal change was observed between the results of the tensile only and the tensile-compressive SRBC elastic fine results. 
THE JOHNS HOPKINS UNIVERSTTY

APPLIED PHYSICS LABORATORY

LAUREL MARYLAND

The final step was the use of the bi-linear elastic-plastic material properties option of SAAS III for an even more exact representation. As would be expected at the temperatures predicted during the reentry, the material did exhibit yielding and underwent some plastic flow. Compressive yield strengths of the FWPF are substantially lower than the tensile yield strengths. The stress results did show a slight reduction in magnitude and the safety factors showed a minor increase. However, as in the previous analyses, an axial compressive failure was still predicted. A tabulation of the results from both of these analyses may be found in Table 15.

For all of the VEEGA side-on stable steep gamma angle analyses, the stress values were somewhat higher than those of the previous Iaunch accident reentry analyses, Reference 74 . The minimum safety factors were also slightly reduced from these previous values. Both conditions were expected due to the more severe thermal environment of the VEEGA reentries. The stress results for the most complex analysis conducted ( $f$ ine SRBC-EPTC) showed only one small area of the aeroshell which possibly would fail under the subject VEEGA temperature loadings. The outer aeroshell wall near the stagnation point showed compressive axial stress safety factors of less than 1.00 at the 1.40 second time. Elements $10,20,30,40,50,60,70$ and 80 of Figure 38 (a single outer wall element thickness from $0^{\circ}$ to $37.5^{\circ}$ for the fine model) were the only elements having axial compressive stress safety factors less than 1.00. A single element thickness is one-tenth of the total LWRHU wall thickness or approximately 0.018 inch. The elements deeper into the wall did not show failure at this reentry time, therefore, it is questionable whether the compressive failure of this row of outer wall elements would cause a catastrophic failure of the LWRHU aeroshell. Under compressive loading, the FWPF ultimate strengths used (based on 1\% strain) should be taken not as an indicator of catastrophic failure, but only as a sign of increased compressive plastic deformation. The "failed" material would tend to remain intact and not allow a release of the insulators and the cladded fuel pellet. Cracks in the FWPF material could be initiated by shear under compressive stresses in the highly heated surface layer and further crack propagation could follow under cyclic thermal loadings. As shown in Figure 37, such a reversal of compressive stresses to tensile stresses was predicted later into the VEEGA reentry due to a reversal of the thermal gradient. The "failed" elements and the underlying elements would then be under tensile loadings and would be more likely to cause a catastrophic failure of the aeroshell.

Therefore, a further analysis was performed to better determine the effect of the "failed" elements at later times into the reentry. In order to check for a compressive stress path which could lead to additional failure under compressive loadings, the subject elements were removed from the fine model and an analysis was performed at 1.60 seconds into the reentry. This analysis showed no further failure of the underlying elements due to axial compressive stresses. All other compressive and tensile stresses at this time showed high safety factors. No immediate stress reversal had occurred in the region. The critical area of the aeroshell does undergo a stress reversal later into the reentry as shown previously. A further analysis was performed 
with the removed elements model at 5.60 seconds into the reentry heat pulse as this appeared to be the time of maximum tensile stresses in the critical region. However, the tensile stresses in this area never rise above 3000 psi and the temperatures at these later times are reduced substantially. Thus, safety factors at the time of peak tensile stress in the critical area were well above tensile stress ultimate limits. Results from these additional analyses show that such a compressive failure near the stagnation point would not cause a catastrophic failure of the LWRHU aeroshell. Therefore, no release of the internals of the LWRHU would be expected due to thermal stress for the side-on stable steep gamma reentry. The shallow gamma $\left(-10^{\circ}\right)$ and medi um gamma $\left(-50^{\circ}\right)$ side-on stable temperature predictions were examined and compared to that of the steep gamma $\left(-90^{\circ}\right)$ temperature predictions. Thermal gradients were lower for these additional analyses and are therefore considered less severe from a thermal stress standpoint. Thermo-structural analyses utilizing these inputs, therefore, were considered unnecessary. These reentries are more important in the ablation analyses.

\section{Results of End-on Stable Analysis}

The steep gamma reentry was again examined first for the end-on stable configuration because of the severity of the heat pulse at this reentry angle. This was the same assumption made in the previous side-on stable reentry analysis. Initial conditions for the steep gamma end-on stable reentry configuration are the same as for the side-on stable: an inertial velocity of 46,750 feet per second, an inertial flight path (gamma) angle of $-90^{\circ}$, and a free release altitude of 271,000 feet.

The approximate thermo-structural response of the aeroshell during the first 6.00 seconds of the reentry heat pulse was determined with the axisymmetric option of SAAS III. Symmetry in the axial direction was assumed, thereby reducing the size and complexity of the model. This assumption is based upon the belief that boundary effects at the closed end of the aeroshell will not alter behavior at the end cap region. Figure 39 shows the end-on geometry and boundary conditions chosen.

A finite element model which consisted of 144 four-noded axisymmetric elements was utilized with six elements through the thickness of the aeroshell wall and six elements through the thickness of the end cap wall. The element and node layout of the end-on model in a rectangular $(R-Z)$ coordinate system is shown in Figure 40. The axisymmetric option of SAAS III considers this geometry to be a solid rotated through $360^{\circ}$ about the $Z$-axis. The side-wall and the end cap of the aeroshell were considered a monolithic structure in the initial analysis executions. Also, all elastic material properties were used in the initial approximations. Use of these initial assumptions provided a quick and inexpensive method of determining the time of maximum thermallyinduced stresses during the reentry.

Temperature distribution data and surface ablation data at discrete time steps into the steep gamma angle reentry were obtained from the SHTPE code. 
THE JOHNS HOPKINS UNIVERSITY APPLIED PHYSICS LABORATORY lauRel maryland

The code provided a stable numerical solution throughout the investigated period of the reentry, unlike the experience for the side-on reentry configuration. With SHTPE data available, shape change of the end cap outer surface would be modeled as well as the temperature distribution over the aeroshell, providing for a more realistic analysis.

The time of maximum temperature was found to be approximately 3.00 seconds with a maximum of $7460^{\circ} \mathrm{R}$ at the center of the outer surface of the end cap. Thermal gradients through the wall of the end cap behaved in a similar manner as those of the side-on configuration. Initially, very large thermal gradients through the wall of the end cap caused high stresses in the surrounding region. These thermal gradients peaked at about 1.60 seconds into the reentry and then diminished steadily. A direction reversal of thermal gradient near the center of the end cap occurred after approximately 4.50 seconds, as with the side-on stagnation point. At the edge of the aeroshell wall, however, this reversal did not occur. The axial thermal path provided by the wall of the aeroshell lessened the effect of the thermal gradient in this area. At the end of the 6.00 second study, elements in this region were nearly the same temperature.

A thermal path was also created radially through the end cap to the aeroshell wall, thus creating high radial thermal gradients in the end cap. An outward bowing of the end cap was evident as it expanded within the constraints of the aeroshell wall. The end cap's expansion contributed to the radial deformation of the aeroshell wall as evidenced early into the reentry.

The radial gradient becomes the driving force in the end-on analysis as did the axial gradient in the side-on case.

Stresses were evaluated throughout the entire 6.00 seconds of the reentry for which thermal response had been derived. The method used for the evaluations was similar to that of the side-on stable analysis. Times of maximum stresses and minimum safety factors were determined. Hoop stress appeared to be the most critical as in the side-on stable analysis. Radial stress was also considered important due to the rapid expansion of the constrained end cap as described above.

Table 16 is a tabulation of the hoop and radial stresses and corresponding factors of safety for the end-on elastic analysis. Axial stress was of little importance due to the long thermal path provided by the side wall of the aeroshell. The maximum compressive hoop stress occurred at 1.00 second at the leading edge of the aeroshell wall. The minimum safety factor for compressive hoop stress occurred at the time of maximum thermal gradient at 1.60 seconds. Tensile hoop stress achieved a maximum at the inner wall of the aeroshell at 2.90 seconds. The maximum value dropped slightly until 5.00 seconds, then rose somewhat. The leading edge of the aeroshell wall showed the minimum safety factor at this time. Tensile and compressive radial stresses achieved maximums at the same time of 1.20 seconds. Magnitudes and 
THE JOHNS HOPKINS UNIVERSITY APPLIED PHYSICS LABORATORY

laupel mapylano

safety factors for the compressive radial stresses follow closely with the compressive hoop stresses. Tensile radial stress safety factors never dropped below a value of 4.0. As stated above, axial stresses were of little concern never dropping below a 2.0 safety factor. Major and minor principal stress levels followed closely with those of the hoop and radial stresses. All safety factors for the end-on elastic material properties analysis showed a minimum margin of safety of at least 50 percent.

Two further analyses were conducted at the 1.60 second time of the reentry. The first was a SAAS III execution with elastic-plastic tensile and compressive material properties. Stress levels were little changed from the elastic tensile-only run indicating a good initial representation of the material in this run. Very few of the elements were in total compression and little, if any, plastic flow had occurred at this critical time of the reentry. Another SAAS III execution was run using gap elements to represent the thread openings of the end cap to aeroshell side wall interface. This procedure was the same as utilized by Waeber in the previous series of LWRHU analyses. As expected, some shifting of stresses to structural elements in the thread area was encountered. Also evident was the reduction of stresses in the aeroshell wall past the end cap, indicating less bending of the aeroshell wall due to the uncoupling of the two pieces. However, no gap openings occurred during this analysis, indicating that the seal between the end cap and the aeroshell wall remained intact. Safety factors for both of the additional runs showed that maximum stresses did not exceed ultimate strengths of the FWPF material. Therefore, based on thermo-structural considerations, the LWRHU aeroshell is expected to survive an end-on stable steep gamma VEEGA reentry intact and without a release of the internal insulators or cladded fuel pellet.

As with the side-on stable analysis, the shallow gamma $\left(-10^{\circ}\right)$ and medium gamma $\left(-50^{\circ}\right)$ end-on stable temperature predictions were examined and compared to that of the steep gamma $\left(-90^{\circ}\right)$ temperature predictions. Thermal gradients were lower for these additional analyses and are therefore considered less severe from a thermal stress standpoint. Thermo-structural analyses utilizing these less severe inputs therefore were considered unnecessary. 
THE JOHNS HOPKINS UNIVERSITY APPLIED PHYSICS LABORATORY LAUAEL MAAYLANo

VIII. LWRHU ABLATION RESPONSE TESTS

\section{A. Test Program}

\section{Summary}

A series of tests was successfully conducted to assess the full scale ablation response behavior of the General Purpose Heat Source (GPHS), the Graphite Impact Shell (GIS), and the Light Weight Radioisotope Heater Unit (LWRHU) modules to simulated reentry environments. The LWRHU was tested in an end-on orientation to (a) eliminate the uncertainties in the aerodynamics and aerothermodynamics that would result from the interference generated by a side-mount, and (b) to minimize the shear load on the model holders. For these first tests, these were considered to be important considerations.

The tests were completed in April 1988 at the NASA/ARC $20 \mathrm{MW} \mathrm{AHF}$ facility. The experimental results are currently being reviewed and analyzed. Prellminary results show that the stagnation point recession of the GPHS (in a broadside orientation) and of the LWRHU (in an end-on orientation) is predicted within $10 \%$ by 1-D predictions for orbital decay conditions. The experimental results will be documented as a) a "quick look" report, where photographic coverage (still photographs) of the stagnation surface will be presented, and b) a final test report, where the information pertaining to the test and experimental data, as well as the data itself, will be presented.

The objective of the test program (Reference 75) is to assess the ablation response of the full scale, flight ready GPHS/GIS/LWRHU modules (with a fuel simulant in place of the nuclear fuel) for simulated conditions corresponding to orbital decay and/or minimum gamma type entries. Here, the ablation response is defined as the aeroshell material removal and associated surface temperature corresponding to the test conditions. Local aeroshell burnthrough and subsequent module behavior are also of interest and were intentionally programmed.

\section{Background}

Initially, discussions were held with NASA Ames Research Center (NASA/ARC) personnel where an agreement was reached for the testing of the GPHS, GIS, and LWRHU modules at NASA (Reference 76). The NASA facility was the only one able to handle the large cross section of the GPHS module while still delivering a reentry type simulation environment. A two phase test program was proposed (Reference 76 ). The first phase was be conducted in the 20 MW Aerodynamic Heating Facility (AHF) where moderate environments corresponding to an orbital decay reentry can be simulated. The second phase will be conducted in the $60 \mathrm{MW}$ Interaction Heating Facility (IHF) where significantly higher heating and pressure environments will be obtainable. The desired test conditions were defined (Reference 77). Design conditions for the GPHS/GIS/LWRHU module holders were specified (Reference 78). The 
GPHS/GIS/LWRHU module holders were designed by JHU/APL (Reference 79) and fabricated by Los Alamos National Laboratory (LANL). A test plan for the 20 MW AHF was prepared (Reference 75). The test plan for the $60 \mathrm{MW}$ IHF will be similar to the $20 \mathrm{MW} A H F$ test plan. The GPHS/GIS/LWRHU module response measurements in both facilities will be similar, permitting an evaluation of the difference in response to the two environments.

B. Thermal Response Predictions for Ablation Tests at NASA-Ames in the 2OMW Arc Jet Facility

1. Summary

In support of the ablation response tests at NASA/ARC, test conditions were derived using computer analyses to simulate the aerothermal environment of an orbital decay reentry for the LWRHU in the end-on stable orientation. Specifically, the conditions simulate the parameters of the reentry that influence ablation and the ablation mechanism on a time averaged basis. The run time for the initial test case is a simulation of the heat pulse interval for orbital decay; it is followed by extended tests with appropriate stops to examine the model until burn through of the aeroshell is achieved (test to failure). The method used to derive the test conditions is described below.

The thermal response predictions consist of the temperature and aeroshell recession history at the stagnation point. These data were obtained by employing a modified version of the CMA digital code and, as such, they represent $1-D$ heat flow results. Details concerning the predictions are given below.

\section{Approach}

The reentry parameters selected for the simulation of ablation and ablation mechanism for the orbital decay reentry were the ratio of heating rate to stagnation enthalpy, $\dot{q} / h_{t}$, and the stagnation pressure, $P_{t}$. The initial test interval is derived from the heat pulse. Subsequent test intervals represent pre-selected values of recession.

For consistency, the breakup conditions that lead to the orbital decay reentry for the GPHS module were assumed as initial conditions for the reentry of the LWRHU. They are:

inertial velocity $=25,067$ feet per second

inertial flight path angle $=-0.7882$ degrees

altitude $=270,000$ feet

Starting with these initial flight conditions, the 3DOF trajectory code was used to generate the basic environmental parameters (trajectory histories for the convective heating raes, free molecular heating rates, total pressure, 
THE JOHNS HOPKINS UNIVERSITY APPLIED PHYSICS LABORATORY

LAUPEl Maryland

enthalpy, etc) that are needed for thermal analyses. Next, the CMA thermal response code version which incorporates Hunter's finite rate chemistry method was used to derive the recession history of the aeroshell at the stagnation point.

Figures 41 through 44 present the analytical data from the 3DOF and CMA simulations that are of interest in the arc jet for simulation of the orbital decay reentry. The heat pulse, illustrated by Figure 41 , includes the effect of Matting's bridging relationship between the continuum and the free molecular flow regimes. After about 160 seconds of flight time, the convective heating rates approach zero. The aeroshell's recession history at the stagnation point of the end-on face is shown by Figure 42. About 20 seconds of reentry time are consumed to increase the surface temperature to a magnitude which causes ablation and the recession is virtually completed after about 160 seconds. From these results, it was concluded that an appropriate test should reproduce the integral of heating rate divided by enthalpy through the 160 second flight period.

The $20 \mathrm{MW}$ arc jet facility does not have the capability to provide a controlled, transient thermal environment; constant test condition measurements are necessary. The main parameters that influence the $r$ ates for ablation during an orbital decay reentry are the total pressure and the ratio of the convection heating rates to the total enthalpy. Histories for these parameters are given by Figures 43 and 44 . Their time averaged values, over the selected segment of the reentry, define appropriate test conditions if the diffusion limited oxidation ablation mechanism is also duplicated. The respective values are 0.0272 atmospheres and 0.00675 pounds mass per square feet-second. According to the test facility personnel, with the total pressure fixed at 0.0272 atmospheres, the convective heating rate was expected to be about 40.52 Btu per square-feet-second (based on the reference one-foot radius sphere) and the total enthalpy was expected to be about 6000 Btu per pound-mass. While a few seconds of test time are needed to warm-up the aeroshell, these test conditions (and a duration of 173 seconds) satisfy the requirement of duplicating the ablation mechanism of diffusion limited oxidation.

\section{Thermal Response Predictions}

The test model differs from the LWRHU in that ATJ graphite was used to simulate the fueled-clad assembly and for mounting the model to the sting. The mounting protrudes approximately 0.6 of an inch into the innards. During testing, the cap end was windward. The thermal model represents a one-dimensional heat flow section taken through the stagnation point. It was solved using a modified version of the CMA code.

Response predictions based on the above test conditions are presented in Figure 45 (the stagnation point temperature history) and Figure 46 (the recession history of the aeroshell at the end-on stagnation point). The stagnation point temperature reaches $2600^{\circ} \mathrm{R}$ in about 20 seconds, $3400^{\circ} \mathrm{R}$ in 
THE JOHNS HOPKINS UNIVERSITY APPLIED PHYSICS LABORATORY

laufel marylano

about 50 seconds, and then, gradually increases to beyond $3600^{\circ} \mathrm{R}$ by a test time of about 600 seconds. The precise time, during the test $r$ un, when the ablation mechanism becomes diffusion limited oxidation is debatable; there is a considerable amount of scatter in the data base. Also, some recession will take place at a slightly lesser rate during the transition period. To accomodate these factors and to allow for warm-up, 13 seconds were arbitrarily added to the test time required to simulate the orbital decay reentry. Figure 46 also shows the amount of test time for the events of $50 \%, 75 \%$ and $100 \%$ burn through of the aeroshell. When the test is restarted, the warm up period is integrated into the predicted test time for the event. In sequential events, the thermal mass of the test model is reduced, but the transition time to reach the ablation temperature range is short since the aeroshell material is a good conductor. Consequently, the prediction test time for a given event should not be too far different from the required constant temperature interval.

\section{Conclusions and Recommendations}

Test conditions were derived to simulate the orbital decay reentry of the LWRHU for the end-on stable orientation. Thermal response predictions were made based on the selected conditions. Owing to the limited time frame prior to testing, simple analytical tools (one-dimensional thermal models) were applied. The results were intended to guide the planning of the program and to serve as a basis for making adjustments between test cases, depending upon previous results. After the initial test case (orbital decay simulation) it was planned that the model would be carefully examined. Factors such as more severe recession rates away from the stagnation point, the influence of surface features on recession rates, and the possibility of mechanical erosion or a turbulent air stream merit special consideration.

It should be noted that the reentry heating rates for the side-on stable orientation case could be more severe (at the stagnation point), than for the end-on case. Due to difficulties in mounting a side-on model without generating unacceptable flow interference, and because interest focused on the nature of a possible failure in the region of the cap, the end-on case was selected.

The test results from the $20 \mathrm{MW}$ arc jet facility will be evaluated in detail and used as a critical input in defining appropriate test conditions for the tests scheduled in the $60 \mathrm{MW}$ facility. Heating rates provided by the 60 MW facility are high enough that graphite sublimation is expected. Although the results may relate to some of the milder Galileo-VEEGA reentries, this test is scheduled beyond the cut of $f$ date for input to the FSAR. 
THE JOHNS HOPKINS UNIVERSITY APPLIED PHYSICS LABORATORY

LAUAEL MAAYLANO

\section{OTHER REENTRY CONDITIONS}

Reentry analysis for other reentry scenarios including parking orbits, minimum gamma entries, and explosions were addressed in Appendix $G$ of Ref erence 80. Except for the explosion scenario, the analyses conducted for this SAR include those conditions both in scope and in modelling the problem. The results, documented in Reference 80 showed that no ablation failure of the LWRHU nor fuel melt is expected of the orbital decay or minimum gamma entries based on the zonal basis approach and other assumptions used herein (multiple skips are not included). The explosion scenario was not addressed herein. The previous SAR (Reference 80) examined an orbital and ascent explosion. This previous study showed that for an assumed bare clad entry, clad melt, but not fuel melt, would occur. 


\section{REFERENCES}

Section I

1. Hagan, J. C., JHU/APL Letter to G. L. Bennett, DoE, "Planning for LWRHU/GPHS Reentry Analysis", ATD-RL-87-071 dated October 21, 1987.

\section{Section II}

2. Johnson, E. W., Light Weight Radioisotope Heater Unit Safety Analysis Report (LWRHU-SAR), Vol. I, Reference Design Document, Monsanto Research Corporation Report MLM-3293, Miamisburg, OH, October 1985.

\section{Section III}

3. McRonald, A., Galileo VEEGA Earth Reentry Breakup Anal ysis, JPL D-4222 (Preliminary Release), Jet Propulsion Laboratory, Pasadena, CA, March 1987 .

4. Letter from MCRonald, A., (Jet Propulsion Laboratory) to Hagan, J. C. (Applied Physics Laboratory), MS-301-165, August 17, 1987.

5. Conn, D. W., "Galileo-VEEGA Failure Boundaries and Related Computational Requir ements", BFD-2-87-017, JHU/APL, Laurel, MD, September 21, 1987.

Section IV

6. Johnson E. W., Light Weight Radioisotope Heater Unit Safety Analysis Report (LWRHU-SAR), Vol. II, Accident Model Document, Appendix G.., Monsanto Research Corporation Report MM-3293, Miamisburg, OH, October 1985.

\section{Section $\mathrm{V}$}

7. Lucero, E. F., "Estimated Continuum and Free Molecular Aerodynamics and Bridging Parameters for the LWRHU," JHU/APL BFD-2-88-004, March 2, 1988.

8. Lucero E. F., "Estimated Subsonic Aerodynamics and Terminal Velocity of the RTG, GPHS Module, GIS, Fuel Clad and LWRHU," JHU/APL BFD-2-87-023, dated December 2, 1987.

Section VI (A. and C.)

9. Conn, D. W., "Reentry Safety Evaluation Process - a Report", ANSP-294, Johns Hopkins University/Applied Physics Laboratory, Laurel, MD, November 12, 1986.

10. Perini, L. L., "User's Manual for the 3DOF Trajectory Computer Program", ANSP-M-6, JHU/APL, Laurel, MD, September, 1973. 
11. Lucero, E. F., "Compilation of Aerodynamic Drag Inputs to the APL 3DOF Reentry Program", BFD-2-88-005, JHU/APL, Laurel, MD, March 2, 1988.

12. Nicolet, W. E., User's Manual for the Generalized Radiation Transfer Code (RAD/EQUIL), Report to UM-69-9, Aerotherm Corporation, Mt. View, CA, October 1, 1969 .

13. Nicolet, W. E., Advanced Methods for Calculating Radiation Transport in Ablation-Product Contaminated Boundary Layers, NASA-CR-1656, Aerotherm Corporation, Mt. View, CA, September 1970.

14. Sutton, K., "Air Radiation Revisited", AIAA Paper 84-1733, AIAA 19th Thermosphysics Conference, Snowmass, CO, June 1984.

15. Olstad, W., "Program to Compute the Inviscid Radiating Flow about a Circular Cylinder", Informal, transmitted from W. Olstad, NASA-Langley Research Center, Hampton, VA, December 13, 1973.

16. Olstad, W. B., "Nongray Radiating Flow about Smooth Symmetrical Bodies", AIAA Journal, Vol. 9., No. 1, pp. 122-130, January 1971.

17. Technical Progress Report No. 134, September 1, 1987 through September 31, 1987, Aerospace Nuclear Safety Program, JHU/APL, Laurel, MD, January 15, 1988 .

18. Detra, R. W., Kemp, N. H. and Riddell, F. R., "Addendum to Heat Transfer to Satellite Vehicles Re-entering the Atmosphere", Jet Propulsion, Vol. 27, No. 12, pp. 1256-1257, December, 1957.

19. Fay. J. A. and Riddell, F. R., "Stagnation Point Heat Transfer in Dissociated Air", Journal of the Aeronautical Sciences, Vol. 25, No. 2, pp. 73-85, February, 1958.

20. Rose, R. H., and Stankevics, H. O., "Stagnation-Point Heat Transfer Measurements in Partially Ionized Air', AIAA Journal, Vol. 1, No. 12, pp. 2752-2763, December, 1963.

21. Perini, L. L., "Compilation and Correlation of Experimental, Hypersonic, Stagnation Point Convective Heating Rates", ANSP-M-4, Johns Hopkins University, Applied Physics Laboratory, Laurel, MD, July 1972.

22. Perini, L. L., "Compilation of Experimental Stagnation Point Velocity Gradients and Heat Transfer Data in Subsonic and Supersonic Flow", ANSP068, Johns Hopkins University Applied Physics Laboratory, Laurel, MD, August 1975. 
23. Sibulkin, M. J., "Heat Transfer Near the Forward Stagnation Point of a Body of Revolution", Journal of the Aeronautical Sciences, Vol. 19, No. 8 , pp. 570-571, August 1952 .

24. Lees, L., "Laminar Heat Transfer Over Blunt-Nosed Bodies at Hypersonic Flight Speeds", Jet Propulsion, Vol. 26, No. 4, pp. 259-269, April 1956.

25. Lundberg, R. E., et al, A Study of the Behavior of Small Spheres During Reentry, SC-CR-67-2635, Aerotherm Corporation, Mt. View, CA, June 1967.

26. Schaaf, S. A. and Talbot, L., Mechanics of Rarefied Gases, Handbook of Supersonic Aerodynamics, Section 16, NAVORD Report 1488 (Vol. 5), February, 1959.

87. Matting, F. W., "Approximate Bridging Relations in the Transitional Regime between Continuum and Free-Molecule Flows", Journal of Spacecraft and Rockets, Vol. 8, No. 1, January 1971.

28. Kemp, N. H., Rose, P. H., and Detra, R. W., "Laminar Heat Transfer Around Blunt Bodies in Dissociated Air", Journal of the Aeronautical Sciences, Vol. 26, No. 7, July 1959.

29. Rabinowicz, J., Aerodynamic Studies in the Shock Tube, Galcit Hypersonic Research Project, Memorandum 38, June 10, 1957.

30. SNAP-27 Safety Report, Volume II - Accident Model Document Supplement No. 1, Appendix P.-Reentry Evaluation of GLFC and LM, DIN:6300-300PRI, Isotope Power systems Operation/Missile and Space Division, General Electric Co., September 16, 1968.

31. Klett, R. D., Drag Coefficients and Heating Ratios for Right Circular Cylinders in Free Molecular and Continuum Flow From Mach 10 to 30 , SC-RR-64-214, Sandia Laboratories, Albuquerque, NM, December 1964.

32. Perini, L. L., "Local Heat Transfer Coefficients Around a Circular Cylinder in Subsonic Flow", ANSP-188, JHU/APL, Laurel, MD, August 27 , 1980.

33. Putz, R. E., and Bartlett, E. P. "Heat Transfer and Ablation Rate Correlations for Reentry Heat Shield and Nosetip Applications", AIAA Paper No. 72-91, AIAA 10th Aerospace Sciences Meeting, San Diego, CA, January 1972.

34. Spalding, D. B., "A Standard Formulation of the Steady Convective MassTransfer Problem", International Journal of Heat and Mass Transfer, Vol. 1. P.192, 1960. 
35. Wittliff, C. E. and Curtis, J. T., Normal Shock Wave Parameters in Equilibrium Air, Report CAL-111, Cornell Aeronautical Laboratory, August 1962 .

36. Equations, Tables and Charts for Compressible Flow, NACA Report 1135, NASA Ames Research Center, 1953.

37. Hilsenrath, J. H., et al, Tables of Thermodynamics and Transport Properties of Air, Argon, Carbon Dioxide, Hydrogen, Nitrogen, Oxygen and Steam, Pergaman Press, NY, 1960.

38. Gregorek, G. M. and Korkan, K. D., "An Experimental Observation of the Mach and Reynolds Number Independence of Cylinders in Hypersonic Flow", AIAA Journal, Vol. 1. No. 1, May 1963.

39. Gowen, F. E. and Perkins. E. W., Drag of Circular Cylinders for a Wide Range of Reynolds Numbers and Mach Numbers, NACA TN 2960, Ames Aeronautical Laboratory, Mof fett Field, CA., June 1953.

40. Lewis, C. H. and Burgess, E. G., Empirical Equations for the Thermodynamic Properties of Air and Nitrogen to 15000K, TDR No. AEDC-TDR63-138, ARO, Inc, Arnold Engineering Development Center, July 1963.

41. Batt, R. G. and Legner, H. H., "A Review of Roughness - Induced Nosetip Transition", AIAA Journal, Vol. 21, No. 1, pp. 282-289, January 1983.

42. Conn, D. W., "Boundary Layer Transition - GPHS Stable Reentries", ANSP240, JHU/APL, Laurel, MD, August 16, 1983.

43. Perini, L. L., Heat and Mass Transfer Correlation Equations for Subliming Graphite in High Speed Flow, ANSP-M-11, Johns Hopkins University Applied Physics Laboratory, Laurel, MD, August 1974.

Section VI. D

44. Johnson, E. W. Light Weight Radioisotope Heater Unit Safety Analysis Report, LWRHU-SAR, Volume II: Accident Model Document, Appendix G: LWRHU Reentry Response, MLM-3293, Mound, Miamisburg, OH, October 1985.

45. Perini, L. L., Review of Graphite Ablation Theory and Experimental Data, ANSP-M-1, JHU APL, Laurel, MD, December 1976.

46. Lundell, J. H. and Dickey, R. R., "Graphite Ablation at High Temperatures", AIAA Paper No. 71-418, AIAA 6th Thermosphysics Conference, Tullahoma, TN, April 1971.

47. Hunter, L. W., "The Ablation Rate of Burning Carbon and In-Depth Heat Flux", ANSP-260, (BBP-84-153), JHU/APL, Laurel, MD, July 27, 1984. 
48. Dolton, T. A., et al, "Thermodynamic Performance of Carbon in Hyperthermal Environments", AIAA Paper No. 68-754, AIAA 3rd Thermosphysics Conference, Los Angeles, CA, June 1968.

49. Putz, K. E. and Bartlett, E. P., "Heat-Transfer and Ablation-Rate Correlations for Reentry Heatshield and Nosetip Applications", AIAA Paper No. 72-91, AIAA 10th Aerospace Sciences Meeting, San Diego, CA, January 1972.

50. User's Manual Aerotherm Charring Material Thermal Response and Ablation Program Version 3: Volume 1: Program Description and Sample Problems, AFRPL-TR-70-92, Air Force Rocket Propulsion Laboratory, Edwards, CA, April 1970.

51. User's Manual, Aerotherm Equilibrium Surface Thermochemistry Computer Program, Version 3; Volume 1: Program Description and Sample Problems, AFRPL-TR-70-93, Air Force Rocket Propulsion Laboratory, Edwards, CA, April 1970.

52. Moyer, C. B. and Rindal, R. A., An Analysis of the Coupled Chemically Reacting Boundary Layer and Charring Ablator, Parts I through VI, NASA CR-1061, Vidya Division of Itek Corporation, June 1968.

53. Conn, D. W., "Perspectives of the Fuel Reentry Problems-ANSP Planning", ANSP-282, JHU/APL, LaureI, MD, March 14, 1986.

54. March, E. B., Thermal and Ablation Response of Small Spherical Particles for Reentry -- Summary Report, SC-RR-68-547, Sandia Laboratories, Albuquerque, NM, October 1968.

55. Conn, D. W., "Pu02 Microsphere Reentry Studies: Thermal Properties", ANSP-275, JHU/APL, Laurel, MD, August 16, 1985.

56. Uzzell, J. C., Jr., Handbook of Thermal Properties for Nuclear Power System Reentry Analysis, ANSP-169, JHU/APL, Laurel, MD, October 1979.

57. Hampel, C. A., ed., Rare Metals Handbook, Robert Krieger Publishing Co., Inc. Huntington, NY., Tables $17.5(p .320)$ and $17.6(p .323), 1971$

58. Gardiner, G., A Critical Survey of Six Thermodynamic and Transport Properties of Air, NWC TP 4997, Naval Weapons Center, China Lake, CA., February 1971.

59. Dusinberre, G. M., Heat-Transfer Calculations by Finite Differences, International Textbook Company, Scranton, PA, May 1961. 
60. Randall, J. D., SHTP-E, A Computer Implemetation of the FiniteDifference Embedding Method of Ablation Analysis, CP-067, JHU/APL, Laurel, MD, May 1978.

Section VI. F.

61. L. L. Perini, "User's Manual For the 3DOF Trajectory Computer Program," ANSP-M-6, Sept. 1973.

62. Lucero, E. F., "Compilation of Aerodynamic Drag Inputs to the APL 3DOF Reentry Program," BED-2-88-005, March 2, 1988.

63. Conn D. W., "Gallieo/VEEGA Failure Boundaries and Related Computational Requirements," BFD-2-87-017, EM-5434, September 21, 1987.

64. Hagan, J. C., "Transmittal of Radiative Heat Transfer Calculations," ATD-RL-87-048, JCH-87-036, August 10, 1987.

65. Nicolet, W. E., "User's Manual for the Generalized Radiation Transfer Code (RAD/EQUIL)", NASA CR-11-6353, October 1969.

66. Call1s, L. B., "Solutions of Blunt Body Stagnation Region Flows With Nongray Emission and Absorption of Radiation by a Time Asymptotic Technique," NASA TRR-299, January 1969.

67. Randall, J. D., SHTPE, A Computer Implementation of the Finite Difference Embedding Method of Ablation Analysis, CP 067, JHU/APL, Laurel, MD, May 1978.

68. Conn, D. W., "Final LWRHU End-on (Two Dimensional) Thermal Analysis for Peak Clad Response," ANSP-201/EM-4985, January 27, 1981.

69. Putz, K. E. and Bartlett, E. P., "Heat Transfer and Ablation Rate Correlations For Reentry Heatshield Nosetip Applications," AIAA Paper No. 7291, AIAA 10th Aerospace Sciences Meeting, San Diego, CA, January 1972.

70. Mickley, H. S., et al, "Heat, Mass and Momentum Transfer for Flow Over a Flat Plate With Blowing or Suction," NACA TN 3208, 1954.

71. Spalding, D. B., "A Standard Formulation of the Steady Convective Mass Transfer Problem," International Journal of Heat and Mass Transfer, Vol. 1,1960 .

72. Lundell J. H. and Dickey, R. R., "Graphite Ablation at High Temperatures," AIAA Paper No. 71-418, AIAA Sixth Thermophysics Conference, Tullahoma, TN, April, 1971. 
THE JOHNS HOPKINS UNIVERSITY APPLIED PHYSICS LABORATORY

LAUREL MAaYlano

\section{Section VII}

73. Waeber, K. R., "Thermal Stress Analysis of the Light Weight Radioisotope Heater Unit Aeroshell," JHU/APL ANSP-206 (BFD-4-81-008), May 18, 1981.

74. Johnson, E. W., Light Weight Radioisotope Heater Unit Safety Analysis Report (LWRHU-SAR), Vol. II, Accident Model Document, Monsanto Research Corporation Report MM-3293, Mi amisburg, OH, October 1985. Appendix G.

\section{Section VIII}

75. Lutz, S. A., "Test PIan for the GPHS/GIS/LWRHU Module Ablation Experiment for the NASA Ames Research Center 20 MW Aerodynamic Heating Facility," JHU/APL BBE/EM-5451/BFD-2-87-026, Dec. 1987.

76. Lutz, S. A., "Trip Report on Visit to NASA Ames Research Center (ARC) to discuss the Aerospace Nuclear Safety Program (ANSP) Module Testing," JHU/APL BBE/EAM-7876, June 1987.

77. Lutz, S. A., "Definition of Test Conditions for the GPHS/GIS/LWRHU Module Ablation Experiments," JHU/APL BBE/EM-5418/BFD-2-87-014, Sept. 1987 .

78. Lutz, S. A. and Drobnick, R. A., "Design Conditions for the GPHS/GIS/LWRHU Module Holders for the NASA Ames Ablation Experiments," JHU/APL BBE/EM-5456/BFD-2-88-002, Jan. 1988.

79. Drobnick, R. A., "Design of Model Holders for Aerospace Nuclear Safety Program Ablation Response Tests," JHU/APL BBE/EM-5471/BFD-2-88-007, April 1988 .

Section IX

80. Johnson, E. W. Light Weight Radioisotope Heater Unit Safety Analysis Report (LWRHU-SAR), Vol: II, Accident Model Document, Monsanto Research Corporation Report MM-3293, Miamisburg, OH, October 1985. Appendix G. 
Estimated Drag Coefficients for Light Weight Radioisotope Heater Unit

A. End-on

$\begin{array}{cc}M & C_{D_{C}} \\ 0 & 1.0 \\ 0.8 & 1.2 \\ 1.0 & 1.4 \\ 1.5 & 1.8 \\ 2.0 & 1.9 \\ 10.0 & 1.9\end{array}$

$$
C_{D F M}=2.95
$$

Equivalent sphere radius, $R_{s}=0.147 \mathrm{ft}$

Exponent $E_{D}$ in Matting's Bridging Equation: $E_{D}=2.20$

Reference area: $\quad S=\frac{\Pi D^{2}}{4}=\frac{\Pi(1.0216)^{2} / 144}{4}$

$$
S=0.00569 \mathrm{ft}^{2}
$$

B. Side-on (taken from 3DOF inputs 10/12/79)

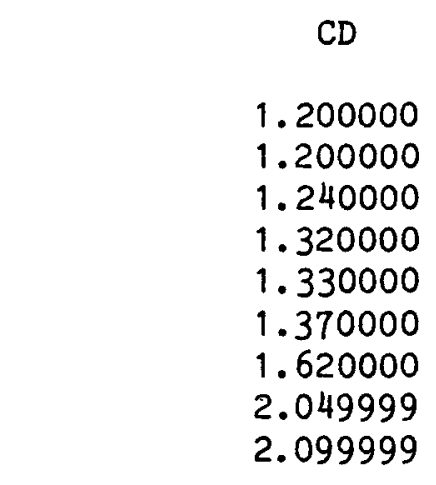

Low Mach No. Table

$C D$

0.554000

0.554000

1.259999

2.000000

2.099999

2.000000

1.699999

1.370000

1.259999

1.240000

1.209999

1.200000

1.200000
Altitude ( $f t$ )

$$
\begin{gathered}
0.0 \\
100000.00 \\
200000.00 \\
230000.00 \\
235000.00 \\
250000.00 \\
300000.00 \\
350000.00 \\
400000.00
\end{gathered}
$$

Switch when $M<0.9$ or when altitude $<100000.0$

$$
\text { Mach }
$$

0.0

0.50

0.60

0.90

1.00

1.10

1.30

1.50

1.80

2.00

2. 30

2.50

40.00

$$
\begin{aligned}
S & =\frac{1.2579 \times 1.0216}{144} \\
& =0.00892 .4 \mathrm{ft}^{2}
\end{aligned}
$$


Estimated Drag Coefficients and Terminal Velocity for the LWRHU.

$$
\text { Axial Crossflow Average }
$$

A. Drag Coefficient

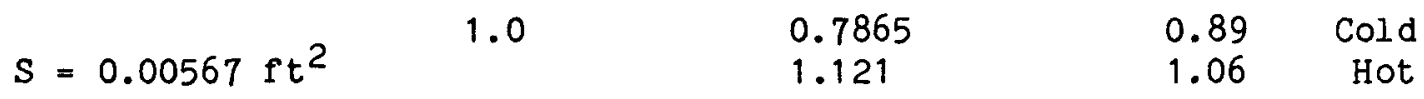

B. $V_{\text {term }}: \mathrm{ft} / \mathrm{sec} /(\mathrm{m} / \mathrm{sec})$

Axial

Crossflow Average

\begin{tabular}{|c|c|c|c|c|c|c|c|}
\hline \multirow{3}{*}{$W=0.08886 \#$} & $\begin{array}{l}\text { Sea } \\
\text { Level } \\
\end{array}$ & $\begin{array}{l}10 \mathrm{~K} \\
\mathrm{ft} \\
\end{array}$ & $\begin{array}{l}\text { Sea } \\
\text { Level } \\
\end{array}$ & $\begin{array}{l}10 \mathrm{~K} \\
\mathrm{ft}\end{array}$ & $\begin{array}{l}\text { Sea } \\
\text { Level } \\
\end{array}$ & $\begin{array}{l}10 \mathrm{~K} \\
\mathrm{ft} \\
\end{array}$ & \\
\hline & $\begin{array}{l}115 \\
(35)\end{array}$ & $\begin{array}{l}134 \\
(41)\end{array}$ & $\begin{array}{l}129 \\
(39)\end{array}$ & $\begin{array}{l}151 \\
(46)\end{array}$ & $\begin{array}{l}122 \\
(37)\end{array}$ & $\begin{array}{l}\left.\begin{array}{l}142 \\
(43)\end{array}\right\} \\
3\end{array}$ & Cold \\
\hline & & & $\begin{array}{l}108 \\
(33)\end{array}$ & $\begin{array}{l}126 \\
(38)\end{array}$ & $\begin{array}{l}112 \\
(34)\end{array}$ & $\left.\begin{array}{l}130 \\
(40)\end{array}\right\}$ & Hot \\
\hline
\end{tabular}


Table 3

Guidelines for LWRHU Reentry Evaluation Process

GALILEO/VEEGA

A. INITIAL REENTRY CONDITIONS:

JPL BREAKUP STUDY FOR GPHS

VELOCITY: 46,750 FPS (EXPECTED)

ALTITUDE: MIDPOINT ON UNCERTAINTY RANGE

GAMMA : $-10,-50,-90$ DEGREES

B. REENTRY CONFIGURATION:

SEQUENTIAL BREAKUP

LWRHU ASSEMBLY $\rightarrow$ CLAD/FUEL ASSEMBLY + EUEL PELLET

NO FUEL PARTICLES OR FRAGMENTS

C. REENTRY ORIENTATION :

ALL CONFIGURATIONS: PRIORITY: 2-D SIDE-ON

SECONDARY: 2-D END-ON

D. FAILURE CRITERIA :

SUBJECTIVE: RECOGNITION OF UNCERTAINTIES

ABLATION: $\quad 50 \%$ AEROSHELL WALL

CLAD MELT: W/INSULATION: EUTECTIC - $300^{\circ} \mathrm{F}$

W/O INSULATION: EUTECTIC $-500^{\circ} \mathrm{F}$

E. BREAKUP CRITERIA :

AEROSHELL: INCIPIENT PENETRATION AT 50\% WALL, STAGNATION REGION CLAD: INCIPIENT MELT, STAGNATION REGION 
GALILEO-VEEGA Reentry Trajectory Analyses Conditions, Assumptions and Limitations

A. Initial Conditions (inertial frame):

\section{VARIABLE}

Velocity (fps)

Flight path (deg)

Altitude ( $f t$ )

Azimuth (deg)

Latitude (deg)

Longitude (deg)
$\underline{A}$

46,750

$-4.5$

330,700

90.0

0.1

0.1

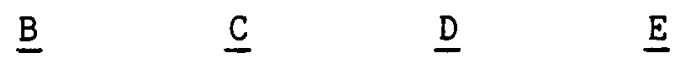

$$
\begin{array}{llll}
-10.0 & -30.0 \quad-50.0 \quad-90.0
\end{array}
$$$$
324,000293,500 \quad 277,000 \quad 271,000
$$

B.

Other Conditions and Assumptions:

Atmos phere: 1962 Standard

Earth Model:

Oblate with polar radius: $20,855,100 \mathrm{ft}$ equatorial radius: $20,925,530 \mathrm{ft}$

Rotating at $0.72921146 \times 10^{-4} \mathrm{radians} / \mathrm{second}$

Boundary Layer is assumed to be laminar.

C. Computer Code Limitations:

No ablation coupling effects on welght or aerodynamics 
Table 5

Galileo VEEGA Reentry Environment Engineering Methods for

Side-On Stable LWRHU Configuration

A. Shock Layer Radi ation

Engineering Method

Feat ures/Discussion

1. Stagnation

Documentation:

Ref. 12 Nicolet, 1969

Ref. $13 ", 1970$

Ref. 14 Sutton, 1984

2. Downstream

Documentation:

Ref. 15 Olstad, 1973

Ref. 16 Olstad, 1971

3. Mass Transfer Blockage

Documentation:

Ref. 17, Progress Report

to DOE \#133
General ized Radiation

Transfer Code (RAD/EQUIL)

reference geometry: sphere

Circular Cylinder Radiating

Flow Field Code

reference geometry: transverse

infinite cylinder

Conn's composite blockage

distribution; Fig. 11 detailed spectral absorption

coefficient model; molecular band, continuum transition and atomic line transport mechanisms; self-absorption, inviscid flow; thermo-dynamic equilibrium; radiative cooling.

- user's manual for RAD/EQUIL code

- theory for transport properties and radiative flux model

- prediction versus ground and flight test data.

six step approximate model for absorption coefficient; steps account for continuum and atomic line radiation; inviscid flow; self-absorption; thermodynamic equilibrium; radiative cooling.

- user's guide for code

- background theory for code; absorption coefficient ul traviolet contribution modified in code.

This effect not yet

incorporated into code.

- compiled blockage data 
Galileo VEEGA Reentry Environment Engineering Methods

$$
\text { for }
$$

\section{Side-On Stable LWRHU Configuration}

\section{B. Convective Heating}

1. Stagnation (a) Hypersonic $\left(M_{\infty}>6\right)$,
Conti nuum flow
Engineering Method

Detra, Kemp and Riddell correlation; reference

geometry; sphere; Figure 12

Feat ures/Discussion

\section{Documentation:}

Ref. 18 Detra, Kemp and Riddell, 1957

Ref. 19 Fay and Riddell, 1958

Ref. 20 Rose and Stark, 1958

Ref. 21 Perini, 1972

(b) Supersonic/Subsonic $\left(M_{\infty} \leqq 6\right)$, conti nuum flow

Documentation

Ref. 22 Perini, 1975

Ref. 23 Sibulkin, 1952

Ref. 24 Lees, 1956
Sibulkin-Lees equation, reference geometry: sphere correlation developed from $\mathrm{F}$ ay and Riddell theory and shock tube heat transfer data; intended range: thermodynamic equilibrium dissociated air; excellent agreement in ionization regime based on shock tube data.

- basic background for correlation

- underlying theory for correlation

- initial exp. data for comparison purposes

- extended exp. data base for comparison; basis for selection; figure 12

combination of Sibulkin theory (incompressible flow) and Lees theory (compressible flow) to cover Mach range; specific heat ratio, $\gamma$, of 1.2 to merge into hypersonic theory.

- development of Sibulkin-Lees equation

- Sibulkin theory

- Lees theory 
Galileo VEEGA Reentry Environment Engineering Methods for

Side-On Stable LWRHU Configuration

B. Convecti ve Heating

(c) Hypersonic, free molecular flow

Documentation

Ref. 25 Kundberg, et al., 1967

Ref. 26, Schaaf and $\mathrm{T}$ al bot

(d) Hypersonic, slip and transition flow

Matting's bridging equation

\section{Documentation}

Ref. 27 Matting, 1971
Engineering Method

$$
\left(\rho_{\infty} v^{3} /(2 J)\right.
$$

Features/Discussion

- assumes unit thermal accommodation coefficient

- background

- theory

semi-empirical engineering relation with foundations from kinetic theory used to bridge heating rates from continuum to free molecular regime for blunt bodies.

- theory and comparison with data 
Galileo VEEGA Reentry Environment Engineering Methods

for

Side-On Stable LWRHU Configuration

B. Convective Heating

Engineering Method

ee's theory; Mach 3.0 laminar heating distribution (Fig. 13)

Documentation:

Ref. 24, Lees, 1956

Ref. 28, Kemp, et al ., 1959

Ref. 29, Rabi nowica, 1957

(b) hypersonic; Leeward

Nestler base heating schedule (Fig. 14)
Features/Discussion

the local to stagnation heating ratio assumed constant over hypersonic flight period; Mach 3.0 distribution selected on the basis of experimental data.

- theory

- experimental distribution data, spheres

- experimental data, cylinders.

used the turbulent branch at central angles > 120 degrees to provide slightly higher heating rates; range of base heating varies from $1 \%$ to $3 \%$ stagnation values

- background on base heating

Document ation:

Ref . 30, SNAP 27, 1968 
Galileo VEEGA Reentry Environment Engineering Methods

for

Side-On Stable LWRHU Configuration

B. Convective Heating

(c) supersonic; windward

(d) supersont c; leeward; continuum

Documentation:

Ref. 31, Klett, 1964

(e) subsonic; windward and leeward; conti nuum

Documentation

Ref. 32, Perini, 1980

(f) hypersonic; free-molecular flow

(g) hypersonic; slip and transsition flow
Engi neering Method

Lee's theory; Mach 2.0 laminar

heating distribution (Fig. 13)

Klett base heating schedule;

Figure 14

Perini's composite heating rate distribution; Fig. 15

$\cos \theta$

Matting's bridging equation
Features/Discussion

heating ratio assumed constant over the range of $M=5$ to $M=1$;

range of base heating varies from $5 \%$ to $9 \%$ stagnation values.

- background on base heating

distribution based on smoothing of a compilation of heating data for subcritical flow; leeward distribution (Fig. 15) rates determined on the basis of $R_{e_{D}}$ variation.

- data compilation, analyses and recommendation.

$\theta$ is central angle measured of $f$ the longitudinal axis (i.e., stagnation location); varies from 1 at stagnation to 0 at 90 degrees.

Re: Section B.1.(d) of this table. 
The Johns Hopkins Uni versity

APPLIED PHYSICS LABORATORY

Laurel, Maryl and

Table 5 (cont)

Galileo VEEGA Reentry Environment Engineering Methods

for

Side-On Stable LWRHU Configuration

B. Convective Heating

Engi neering Method

Feat ures/Discussion

3. Mass Transfer Blockage

primary: Putz-Bartlett corr. secondary: Mickley-Spal ding

correlation, Fig. 11.b

Documentation

Ref. 33 Putz-Bartlett, 1972

Ref. 34 Spalding, 1960
ANSP $-M-19$

October 1988 use of Putz-Bartlett produced unstable solutions for steep reentries; resorted to MickleySpalding blowing effect.

- basis for correlation

- basis for correlation 
Table 5 (cont)

Galileo VEEGA Reentry Environment Engineering Methods

for

Side-On Stable LWRHU Configuration

1. Stagnation:
(a) pressure; hypersonic
$\left(M_{\infty} \geqq 5.0\right)$;

\section{hypersonic approximation} $\left(\gamma P_{\infty} M_{\infty}{ }^{2}\right)$ af $t$ of shock

Document ation.

Ref. 35, Wittliff and Curtis, 1962

(b) pressure; supersonic

$$
\left(5.0>M_{\infty} \geqq 1\right)
$$

normal shock wave equation

Documentation

Ref. 36, NACA, Rpt. 1135, 1953

Ref. 10, Perini, 1973

Ref. 37, Hilsenrath, et al., 1960

(c) pressure; subsonic

(d) enthalpy; all velocity regimes

\section{Documentation}

Ref. 36

isentropic, compressible flow equation specific heat ratio, $\gamma$, evaluated at 1.35 to provide close comparison with real gas normal shock tables

- real gas normal shock parameters.

empirical curve fits for specific heat ratio, $\gamma$, over various temperature ranges

- theory

- curve fits for $\gamma$

- air tables; basis for curve fits

perfect gas specific heat ratio, $\gamma=1.4$.

energy equation: $h+v^{2} /(2 g J)$

- Inviscid, adiabatic gas flow

- theory 


\section{Galileo VEEGA Reentry Environment Engineering Methods}

for

Side-On Stable LWRHU Configuration

C. Flow Field Parameters

(e) velocity gradients

Documentation

Ref. 22, Perini, 1975

2. Downstream

(a) pressure; hypersonic

Document ation

Ref. 38, Gregorek and Korkan, 1963

(b) pressure; supersonic and subsonic

Documentation

Ref. 39, Gowen and Perkins, 1953

(c) enthalpy; all velocity regimes; wi ndward

Documentation

Ref. 40, Lew1s and Burgess, 1963

Engineering Method

Feat ures/Discussion

curve fits to experimental data for spheres and cylinders; Figure 16

Gregorek and Korkan correlation

experimental static pressure distribution

isentropic expansion from stagnation state to local state driven by pressure distributions used to convert stagnation, convective heating rates from reference spherical geometry to the LWRHU.
- theory; experimental data; curve fits
- source, experimental data

- Isentropic expansion using eqilibrium (real gas) thermodynamic model for air.

- thermodynamic model for air 
1. Boundary Layer Transition, (a) nose tip, blunt body

Documentation

Ref . 41, Batt and Legner, 1983

Ref. 42, Conn, 1983
Batt and Legner correlation

rough surface, blunt body transition model

- development of correlation model; survey of boundary layer transition

- background application, GPHS reentries 
Gal il eo-VEEGA Reentry Environmental Parameters

LWRHU Side-On Stable Attitude

Reentry Zones ${ }^{1}$

\section{Reentry Parameters}

1. Max stag. heating rate (BTU $\mathrm{ft}^{-2} \mathrm{sec}^{-1}$ )
(a) convective ${ }^{2}$
(b) radiative 3
(c) flight time, sec

2. Total heat load (BTU $\mathrm{ft}^{-2}$ )
(a) convective
(b) radiative

3. Heat load to $M=5$
(a) convective
(b) radiative
(c) al ti tude at $M=5$

4. Flight time (sec)
(a) hypersonic $(M>5)$
(b) supersonic $(5 \geq M \geq 1)$
(c) subsonic $(M<1)$
A $\left(-4.5^{\circ}\right)$
B $\left(-10^{\circ}\right)$

C $\left(-30^{\circ}\right)$

D $\left(-50^{\circ}\right)$

$E\left(-90^{\circ}\right)$

$\begin{array}{rrrrr}1528.0 & 3018.3 & 5387.0 & 6869.5 & 8382.1 \\ 11.5 & 91.5 & 553.9 & 1054.3 & 1781.1 \\ 36.0 & 16.2 & 5.4 & 3.4 & 2.6\end{array}$

106614.0

48830.0

643.0

28539.0
1208.0

23436.0

1584.0

21729.0

346.0

106439.0
346.0
154938.0
48719.0
643.0
133020.0
28488.0
1208.0
105128.0
23394.0
1584.0
95501.0

21693.0

2097.0

89830.0 
Galileo-VEEGA Reentry Environmental Parameters

LWRHU Side-On Stable Attitude

Reentry Parameters

5. Max stag pressure (atm)

(a) value

(b) fight time, sec

6. Max deceleration

(a) value (gees)

(b) flight time, sec

7. Impact velocity, fps

$$
\begin{aligned}
& 0.079 \\
& 47.0
\end{aligned}
$$

0.499

22.1

$$
\begin{aligned}
& 1.777 \\
& 7.4
\end{aligned}
$$

4.6

$$
\begin{array}{r}
11.5 \\
47.0
\end{array}
$$$$
67.4
$$$$
22.0
$$

237.2

$$
7.4
$$

348.2

4.6

525.5

123.5

123.5

123.5

123.5

124.4

Notes:

1. each zone represented by singular reentry as specified by initial flight path angle in parentheses.

2. stagnation convective heating rates and heat loads based on transformed values from a reference one-foot body sphere to the side-on LWRHU using hypersonic stagnation velocity gradient parameters.

3. stagnation radiative heating rates and heat loads based on an effective spherical radius that provides an equivalent adiabatic shock standof $f$ distance as the side-on LWRHU. 
THE JOHNS HOPKINS UNIVEASITY APPLIED PHYSICS LABORATORY LAUREL MAAYLAND

\section{Table 7}

Initial Radial Temperature Distribution* for Side-on Stable LWRHU Reentries

\author{
Component \\ A eroshell \\ Outer sleeve \\ Middle sleeve \\ Inner sleeve \\ Clad \\ Fuel
}

Temperat ure, ${ }^{\circ} \mathrm{R}$

560.0

780.0

875.0

1105.0

1405.0

.1410 .0

* note: based on steady state radiation to space environment. 
Thermal Properties for FWPF Carbon-Carbon

(Table C-1-2, Ref. 56)

HM PAN FWFF 3OCC,X UIF K:UHUATED, AVG. OATA FK ANSP 167

UPUATE UF BJE.CONN. IOATA (F WPFXY) VIA E=F (T), WEI/ORNL UATED $1 / 8 / 80$

$\begin{array}{rl}460.0 & 20.98 \\ 710.0 & 26.94 \\ 960.0 & 34.77 \\ 1210.0 & 39.57 \\ 1460.0 & 43.16 \\ 1710.0 & 40.76 \\ 1960.0 & 49.16 \\ 2210.0 & 51.56 \\ 2460.0 & 53.96 \\ 2960.0 & 57.55 \\ 3460.0 & 01.15 \\ 3960.0 & 63.55 \\ 4460.0 & 05.95 \\ 5460.0 & 60.91 \\ 7960.0 & 05.90 \\ 8960.0 & 53.40\end{array}$

(1)
(2)

$$
\begin{array}{r}
100.00 \\
85.83 \\
73.33 \\
64.17 \\
56.67 \\
50.83 \\
45.83 \\
41.67 \\
38.33 \\
33.33 \\
30.00 \\
28.33 \\
27.50 \\
25.00 \\
25.00 \\
25.110
\end{array}
$$

- 849

.811

.814

.817

.820

. 823

.826

.829

.833

.840

.848

.857

- 305

- 9ó 7

.948

.948

(4)
.809

.811

.814

.817

.820

- 223

- 826

.829

.833

.840

- 848

. 857

.865

- 837

.948

.948

(5)

Column 1 temperature, ${ }^{\circ} \mathrm{R}$

2 product of density and specific heat Btu $\mathrm{ft}^{-3} \mathrm{R}^{-1}$

3 thermal conductivity, Btu $\mathrm{ft}^{-1} \mathrm{hr} \mathrm{r}^{-1} \mathrm{R}^{-1}$

4,5 emissivity, absorptivity 
Table 8.b

Thermal Properties of Pyrolytic Graphite; C-plane

(LWRHU Radial) Direction (Table G-6-1, Ref. 56)

YRO GRAFHITE-C PLANE; K, FHOCP LASLIR. MULFORDI, E GPAS TASK FORCE

TEMP, R

672.0

1032.0

1392.0

1752.0

2112.0

2472.0

2832.6

3192.0

$3552 \cdot 0$

3912.0

4272.0

$5892 . U$

6892.0

$7 \bullet 42.0$ RHO-CP

30.73

43.05

50.70

55.29

50.03

00.09

62.14

04.48

07.00

09.59

71.47

02.00

02.00

02.10
$K$

1.01

1.03

0.90

0.72

0.57

0.43

J. 44

0.44

0.44

0.44

0.44

0.44

0.44

0.44
EMISS

0.42

0.45

0.47

0.50

0.53

0.55

$0.5 y$

0.01

0.03

0.05

$0.0 y$

0.811

0.80

U. 01$)$
ABSORB

0.42

0.45

0.47

0.50

0.53

0.55

0.58

0.61

0.63

0.06

0.69

0.80

$0.8 v$

0.00 
Thermal Properties for Pyrolytic Graphite, A-B Plane

(LWRHU Circumferential) Direction

(Table G-2-3, Ref. 56)

YROLYTIC GRAPHITE- AB PLANI
TEMP(R)

460.0

660.0

760.0

960.0

1460.0

1960.0

2460.0

2960.0

3460.0

3960.0

4260.0

4460.0

5460.0

6460.0

7460.0
RHO-CP
23.457

.30 .318

.33 .749

.40 .610

.51 .616

.56 .873

60.021

62.972

66.416

69.927

71.411

72.692

79.192

85.692

92.192
RHOCP,K VIKING FSAR,E AB FROM PYROGEN

EMISS

0.80

0.80

0.80

0.80

0.80

0.80

0.80

0.80

0.80

0.88

0.93

0.95

0.95

0.95

0.95
ABSORBTIVITY

0.80

$\overline{0} .80$

0.80

0.80

0.80

0.80

0.80

0.80

0.80

0.88

0.93

0.95

0.95

0.95

0.95 
Table 8.d

Thermal Properties for Pt30Rh

(Table P-2-1, Ref. 56)

PLATINUM ALLOY PT3ORIH; REF: RHUCP,K,E VIA ORINL LETTER 2-23-79

TEMP (R)

540.0

720.0

1250.0

1620.0

1980.0

2340.0

2700.0

3060.0

3420.0

3780.0

4140.0

5000.0

600.100
KחO-CP

39.68

41.23

$44 \cdot 24$

46.41

48.70

50.56

52.01

54.13

55.62

57.10

5 ה.

So.

5d.
$K$

32.95

32.95

37.57

41.62

44.51

47.40

49.71

51.73

53.75

55.78

$57.8 i)$

57.83

51.30
EMISS

0.2

0.2

0.2

0.2

0.2

0.2

0.2

0.2

0.2

0.2

0.2

0.2

0.2
ABSORBTIVITY

0.2

0.2

0.2

0.2

0.2

0.2

0.2

0.2

0.2

0.2

0.2

0.2

0.2 
Table $8 . e$

Thermal Properties for Plutonia

(Table 3, Ref. 55).

WRHU PPO FULL $(87.4 \%)$ : GALILEU FSAH (VOLI, APP A) FUR RHOCP,K; LASL FOR EMISS

TEMP,K

SOU. 0

1032.0

1392.0

1752.0

2112.0

2472.0

2960.0

$346 \mathrm{~J} . \mathrm{U}$

$390 \mathrm{~J} . \mathrm{U}$

$446 \mathrm{~J} .0$

4812.0

4312.01

4813.0

4913.01

$500 \mathrm{~J} \cdot 0$

6000.0

7001.1

$$
\text { RHO-LP }
$$

38.20

38.20

46.83

50.02

50.52

51.08

52.40

53.18

53.95

54.73

55.28

$5 \overline{0} \bar{c} d$

5b. 2त

52.25

35.24

$55 \cdot 20$

$7=. C^{4}$ $x$

2.873

2.87 .3

2.119

1.940

1.337

1.234

1. 153

1.070

U. 987

0.903

0.045

0.845

0.045

11.545

0.145

v.ri4

1. 140
EIISS

0.579

0.643

0.676

0.709

0.751

i. 775

0.819

0.565

0.911

0.557

0.969

0.939

0.119

0.989

0.989

0.

0.119
ABSOKB

0.599

0.643

0.676

0.709

0.751

0.775

0.819

0.805

0.911

0.957

0.985

0.909

0.939

0.789

0.909

0.9 .99

1.949 
Thermal Properties for Plutonia, Heat of Fusion Effect

Incorporated at Melt Failure Threshold ( $\mathrm{T}_{\text {melt }}{ }^{-500}$ )

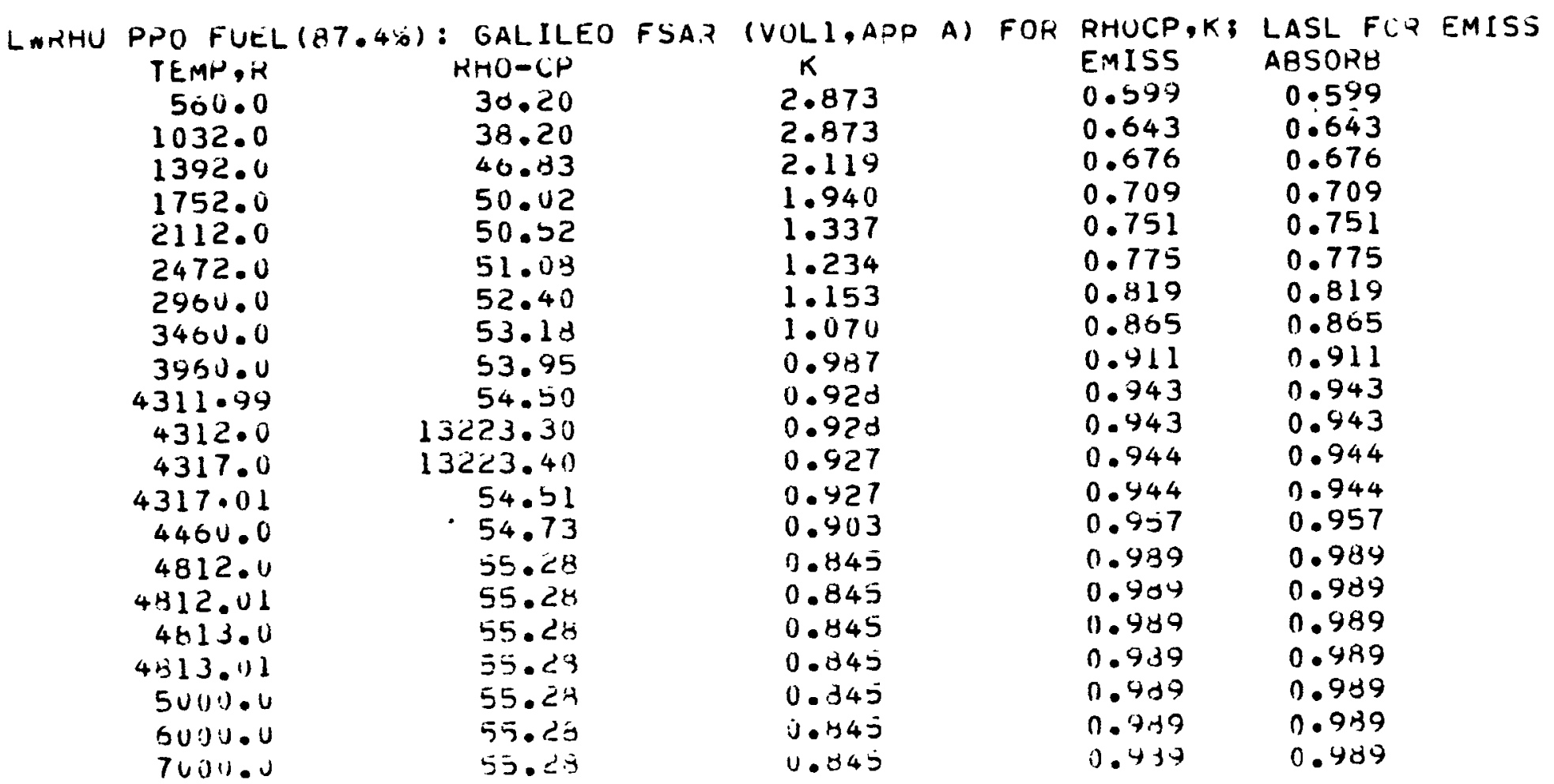




\section{Table 9}

Galileo/VEEGA Reentry Release Conditions Clad/Fuel Assembly

\begin{tabular}{|c|c|c|c|}
\hline & & & \\
\hline VARIABLES & ZONE A & ZONE B & ZONE C \\
\hline Velocity, fps & 28,720 & 19,896 & 1,241 \\
Altitude, ft & 234,419 & 159,323 & 93,732 \\
Flight path angle, deg & +0.32 & -8.69 & -37.04 \\
Clad temperature, F & 2415 & 1074 & 1095 \\
& & & $958-966$ \\
\hline
\end{tabular}




\begin{tabular}{|c|c|c|}
\hline VARIABLE & ZONE A & ZONE B \\
\hline & 28688 & 19547 \\
Velocity, fps & 234457 & 158359 \\
Altitude, ft & +0.325 & -8.70 \\
Flight path angle, deg & $1619-1638$ & $933-988$ \\
Fuel* temperature, & 1538 & $924-928$ \\
Fuel temperature & & \\
Inner array, ${ }^{\circ} \mathrm{F}$ & & \\
\hline
\end{tabular}

* Refer to Fig. 17.b thermal model 
LWRHU End-on Reentry Analysis

Initial (Inertial) Conditions for 3DOF Trajectory Analysis

For all cases: initial velocity $=46,750 \mathrm{fps}$

Azimuth $=90^{\circ} \quad$ (downwind)

Reentry Angle $\quad$ Altitude

Steep Reentry:

$90^{\circ}$

$271,000 \mathrm{ft}$

Shall ow Reentry:

$10^{\circ}$

$324,000 \mathrm{ft}$ 
aeroshell/outer sleeve standoffs - radial

aeroshell/insulator plugs - radial

aeroshell standoff/insulation plug - axial

aeroshell end cap standoff/insulation plug - axial

Contact Conductance, $G=500 \mathrm{Btu} / \mathrm{ft}^{2} \mathrm{hrR}\left(2840 \mathrm{~W} / \mathrm{m}^{2} \cdot \mathrm{R}\right)$ plus radiation

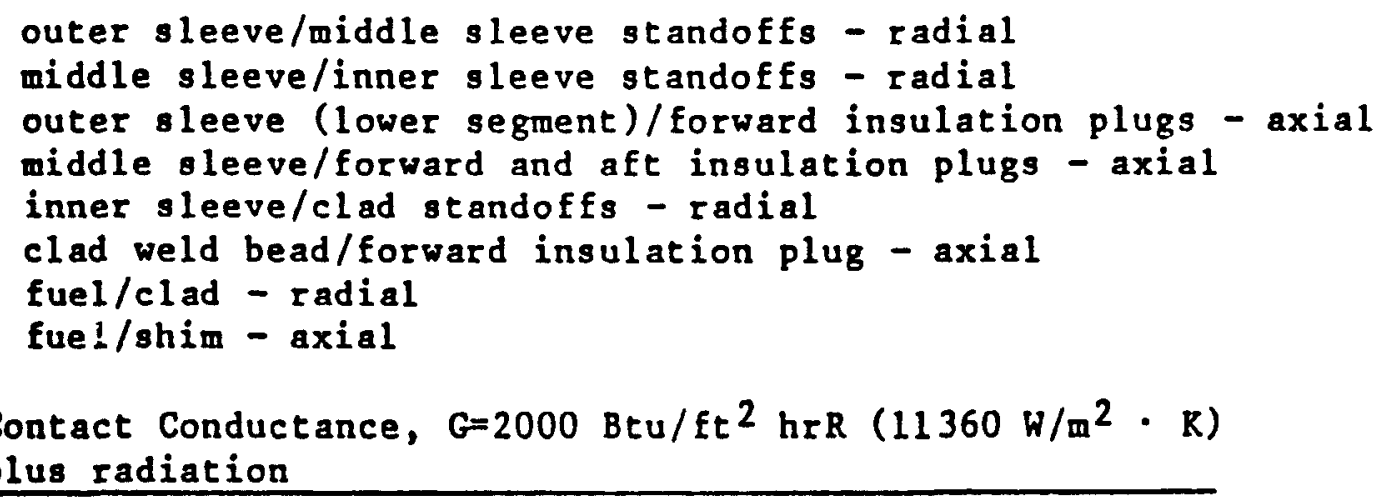

shim/clad (force fit) - radial 
TABLE 13

LWRHU SIDE-ON STABLE COARSE MODEL VEEGA STEEP REENTRY HOOP AND AXIAL STRESSES AND SAFETY FACTORS

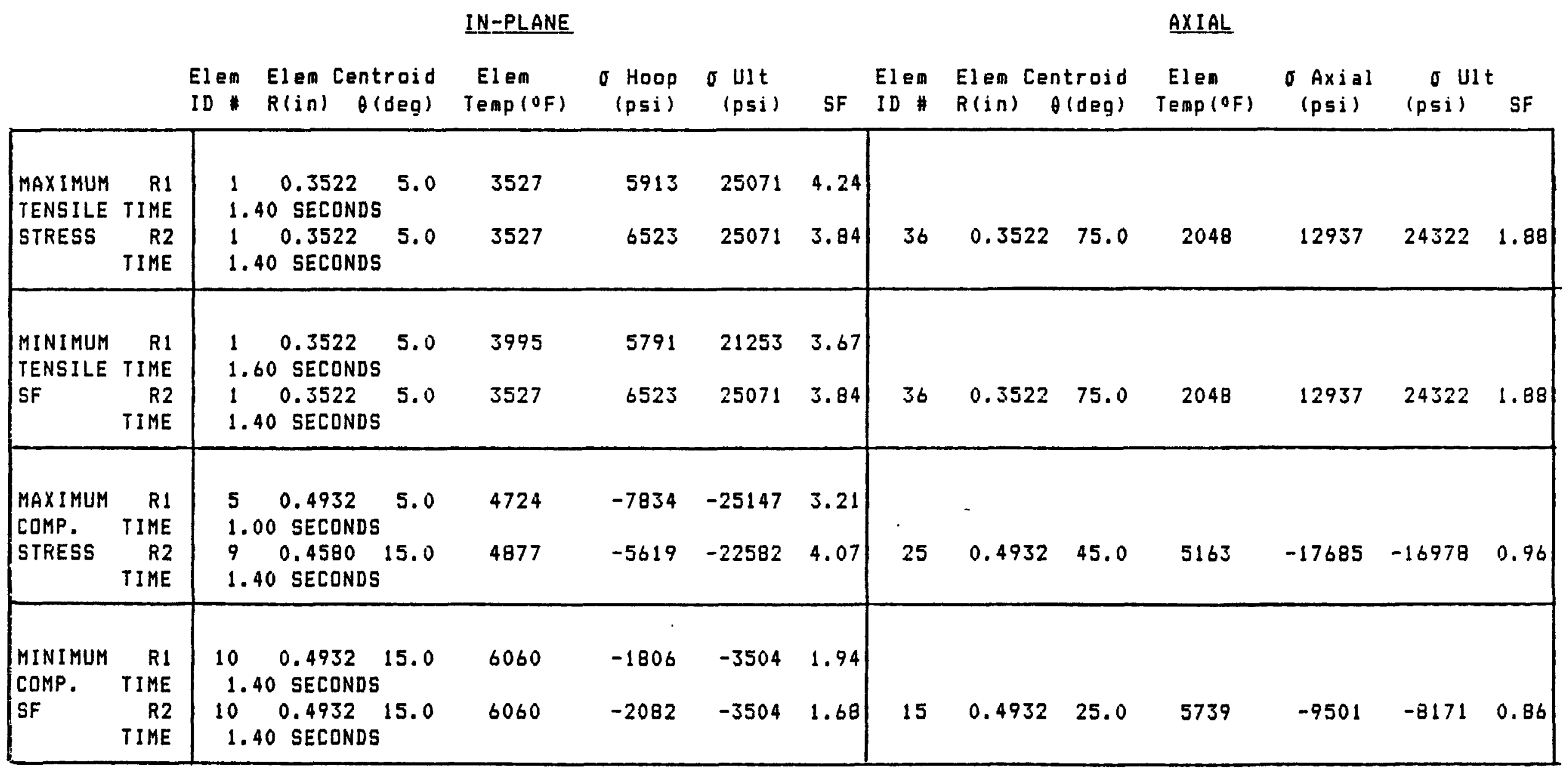

Velocity $=46750 \mathrm{FPS}$, Gamma $=-900$, Altitude $=271000 \mathrm{FT}$ 
TABLE 14

LWRHU SIDE-ON STABLE FINE MODEL VEEGA STEEP REENTRY HOOP AND AXIAL STRESSES AND SAFETY FACTORS AT 1.40 SECDNDS Basic Assumptions

\begin{tabular}{|c|c|c|c|c|c|c|c|c|c|c|c|c|c|c|c|}
\hline & & \multicolumn{7}{|c|}{ IN-PLANE } & \multicolumn{7}{|c|}{$\underline{A X I A L}$} \\
\hline & & $\begin{array}{l}\text { Elem } \\
\text { ID \# }\end{array}$ & $\begin{array}{l}\text { Elem Ce } \\
R(\text { in })\end{array}$ & $\begin{array}{l}n t r o i d \\
\theta(d e g)\end{array}$ & $\begin{array}{c}\text { Elem } \\
\text { Temp (OF) }\end{array}$ & $\begin{array}{l}\sigma \text { Hoop } \\
\text { (psi) }\end{array}$ & $\begin{array}{l}0 \text { U1t } \\
\text { (psi) }\end{array}$ & SF & $\begin{array}{l}\text { Elen } \\
\text { ID }\end{array}$ & $\begin{array}{l}\text { Elem Cer } \\
R(i n)\end{array}$ & $\begin{array}{l}\text { ifroid } \\
\text { (deg) }\end{array}$ & $\begin{array}{l}\text { Elen } \\
\text { Temp (DF) }\end{array}$ & $\sigma_{(p s i)}^{A x i a l}$ & $\underset{(p 5 i)}{\sigma U 1}$ & SF \\
\hline $\begin{array}{l}\text { MAX IMUM } \\
\text { TENSILE } \\
\text { STRESS }\end{array}$ & $\begin{array}{l}\text { R3 } \\
\text { R4 }\end{array}$ & 1 & $\begin{array}{l}0.3334 \\
0.3434\end{array}$ & $\begin{array}{l}2.5 \\
2.5\end{array}$ & $\begin{array}{l}3480 \\
3480\end{array}$ & $\begin{array}{l}5166 \\
5652\end{array}$ & $\begin{array}{l}25365 \\
25365\end{array}$ & $\begin{array}{l}4.91 \\
3.48\end{array}$ & 131 & 0.3434 & 67.5 & 2216 & 12784 & 24290 & 1.90 \\
\hline $\begin{array}{l}\text { MINIMUM } \\
\text { TENSILE } \\
\text { SF }\end{array}$ & $\begin{array}{l}\text { RJ } \\
\text { R4 }\end{array}$ & 1 & $\begin{array}{l}0.3434 \\
0.3434\end{array}$ & $\begin{array}{l}2.5 \\
2.5\end{array}$ & $\begin{array}{l}3480 \\
3480\end{array}$ & $\begin{array}{l}5166 \\
5652\end{array}$ & $\begin{array}{l}25365 \\
25365\end{array}$ & $\begin{array}{l}4.91 \\
4.48\end{array}$ & 131 & 0.3434 & 67.5 & 2216 & 12784 & 24290 & 1.90 \\
\hline $\begin{array}{l}\text { MAXIMUM } \\
\text { COMP. } \\
\text { STRESS }\end{array}$ & $\begin{array}{l}\text { R3 } \\
\text { R4 }\end{array}$ & 9 & $\begin{array}{l}0.4844 \\
0.4844\end{array}$ & $\begin{array}{l}37.5 \\
37.5\end{array}$ & $\begin{array}{l}5235 \\
5235\end{array}$ & $\begin{array}{l}-5568 \\
-6252\end{array}$ & $\begin{array}{l}-16537 \\
-16537\end{array}$ & $\begin{array}{l}2.97 \\
2.64\end{array}$ & 140 & 0.5012 & 67.5 & 4302 & -20007 & $-2 \Xi 808$ & 1.19 \\
\hline $\begin{array}{l}\text { MINIMUM } \\
\text { CDMP. } \\
\text { SF }\end{array}$ & $\begin{array}{l}R 3 \\
R 4\end{array}$ & $\begin{array}{l}10 \\
10\end{array}$ & $\begin{array}{l}0.50 .12 \\
0.5012\end{array}$ & $\begin{array}{l}37.5 \\
37.5\end{array}$ & $\begin{array}{l}6034 \\
6034\end{array}$ & $\begin{array}{l}-2131 \\
-2426\end{array}$ & $\begin{array}{l}-3495 \\
-3495\end{array}$ & $\begin{array}{l}1.64 \\
1.44\end{array}$ & 50 & 0.5012 & 22.5 & 6477 & -4881 & -3514 & 0.72 \\
\hline
\end{tabular}

Velocity $=46750 \mathrm{FPS}$, Gamma $=-900$, Altitude $=271000 \mathrm{FT}$ 
TABLE 15

LWRHU SIDE-ON STABLE FINE MODEL VEEGA STEEP REENTRY HDOP AND AXIAL STRESSES AND SAFETY FACTORS AT 1.40 SECONDS Refined Assumptions

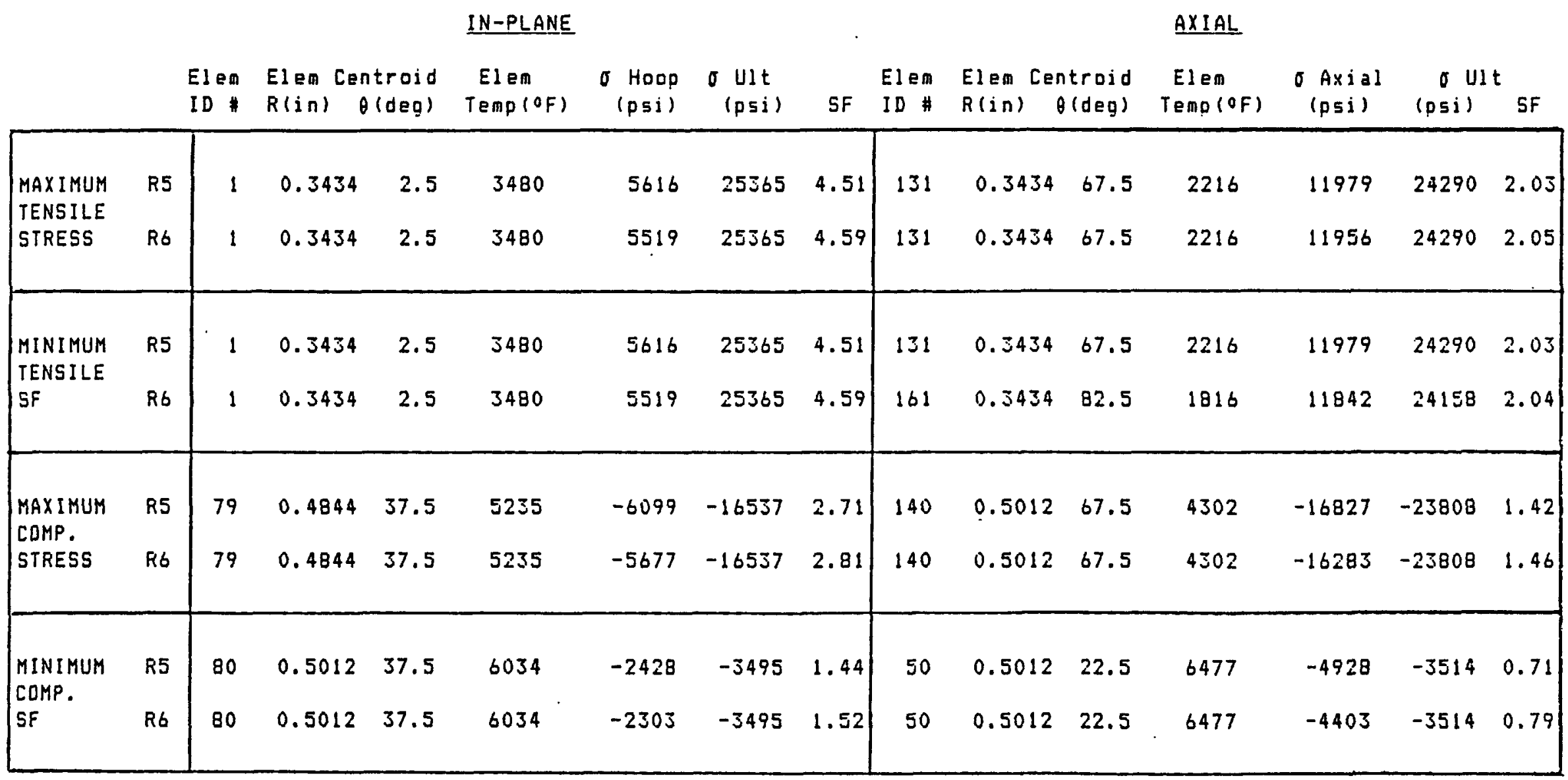

Velocity $=46750 \mathrm{FPS}$, Gamma $=-900$, Altitude $=271000 \mathrm{FT}$ 
THE JOMNS MOTKINS UNIVEASITY

APPLIED PHYSICS LABORATORY LUUREL MaATLAND

TABLE 16

LWRHU END-ON STABLE VEEGA STEEP REENTRY HODP AND RADIAL STRESSES AND SAFETY FACTORS

(ELASTIC TENSILE MATERIAL PROPERTIES)

HOOP STRESS

RADIAL STRESS

\begin{tabular}{|c|c|c|c|c|c|c|c|c|c|c|c|c|c|}
\hline & & & $\begin{array}{l}\text { Elem Centroid } \\
R(i n) \quad I(i n)\end{array}$ & $\begin{array}{c}\text { Elem } \\
\text { Temp (OF) }\end{array}$ & $\begin{array}{l}\sigma \text { Hoop } \\
(p \leq j)\end{array}$ & $\begin{array}{l}\sigma u J t \\
(p s i)\end{array}$ & $S F$ & $\begin{array}{l}\text { Elem } \\
\text { ID } \#\end{array}$ & $\begin{array}{l}\text { Elen Centroid } \\
R(i n) \quad Z(i n)\end{array}$ & $\begin{array}{c}\text { Elem } \\
T \text { Temp }(O F)\end{array}$ & $\begin{array}{c}\sigma \text { Radial } \\
(p s i)\end{array}$ & $\begin{array}{l}\sigma \text { Ult } \\
(p s i)\end{array}$ & $S F$ \\
\hline $\begin{array}{l}\text { MAXIMUM } \\
\text { TENSILE } \\
\text { STRESS }\end{array}$ & TIME & $\begin{array}{r}43 \\
2\end{array}$ & $\begin{array}{l}0.34930 .2485 \\
90 \text { SECONDS }\end{array}$ & 3563 & 14663 & 24780 & 1.69 & 141 & $\begin{array}{l}0.02680 .086 \\
1.20 \text { SECDNDS }\end{array}$ & 3126 & 5724 & 26044 & 4.55 \\
\hline $\begin{array}{l}\text { MINIMUM } \\
\text { TENSILE } \\
\text { SF }\end{array}$ & TIME & 90 & $\begin{array}{l}0.49610 .018 \\
00 \text { SECONDS }\end{array}$ & 4475 & 11479 & 17333 & 1.51 & 141 & $\begin{array}{l}0.02680 .086 \\
1.20 \text { SECDNDS }\end{array}$ & 3126 & 5724 & 26044 & 4.55 \\
\hline $\begin{array}{l}\text { MAXIMUM } \\
\text { COMP. } \\
\text { STRESS }\end{array}$ & TIME & $\begin{array}{r}90 \\
1\end{array}$ & $\begin{array}{l}0.49610 .018 \\
00 \text { SECOND }\end{array}$ & 4587 & -15138 & -27250 & 1.80 & 121 & $\begin{array}{l}-0.18740 .018 \\
1.20 \text { SECONDS }\end{array}$ & 4749 & -11019 & -24793 & 2.25 \\
\hline $\begin{array}{l}\text { MINIMUM } \\
\text { COMP. } \\
\text { SF }\end{array}$ & TIME & $\begin{array}{r}91 \\
1\end{array}$ & $\begin{array}{l}0.32120 .018 \\
60 \text { SECONDS }\end{array}$ & 5762 & -4693 & -7321 & 1.56 & 91 & $\begin{array}{l}0.32120 .018 \\
1.60 \text { SECONDS }\end{array}$ & 5762 & -4725 & -7321 & 1.55 \\
\hline
\end{tabular}

Velocity $=46750$ FPS, Gamma $=-900$, Altitude $=271000 \mathrm{FT}$ 


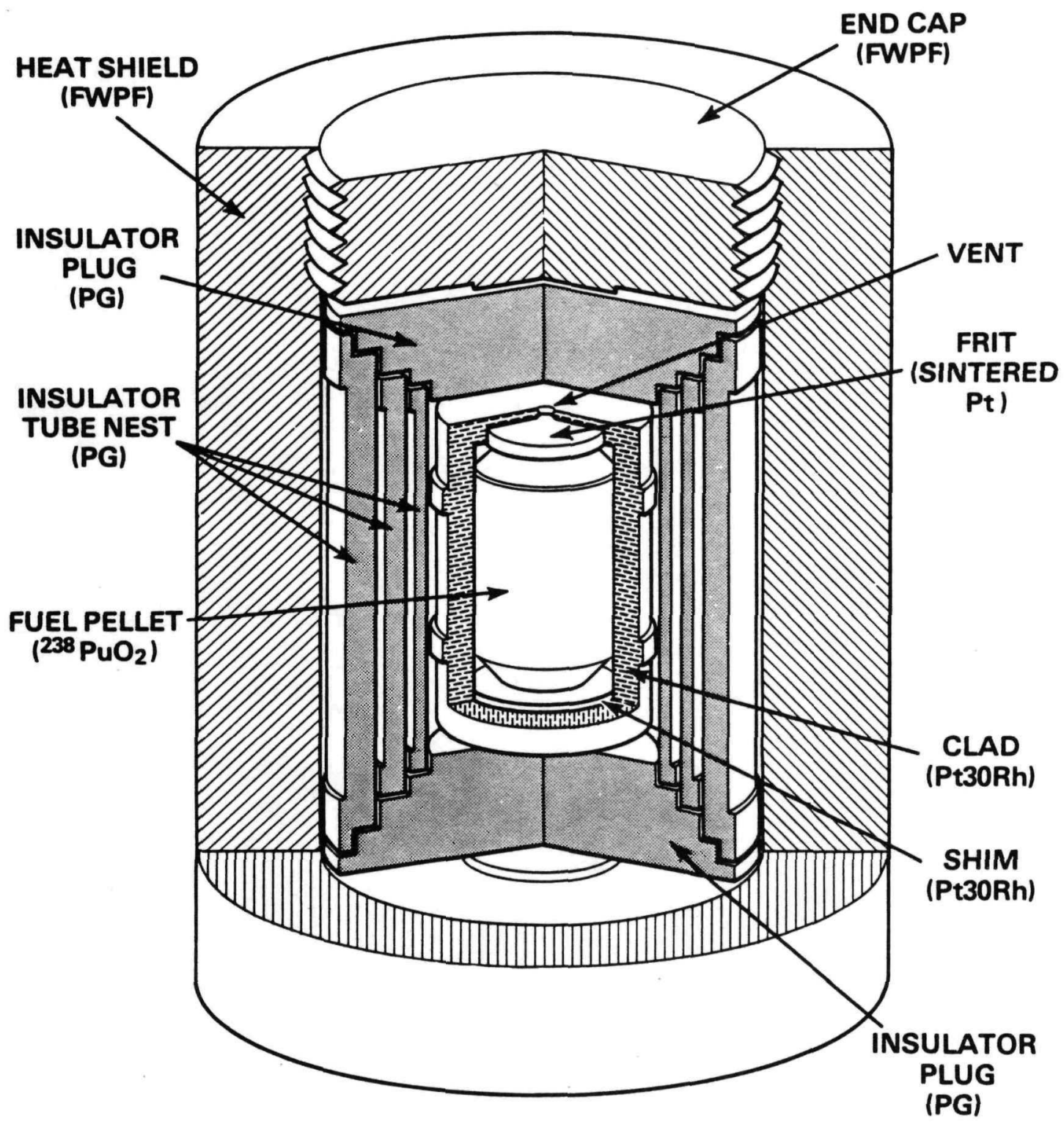

Fig. 1 Cross-sectional view of LVRHU assembly (from Ref. 2). 


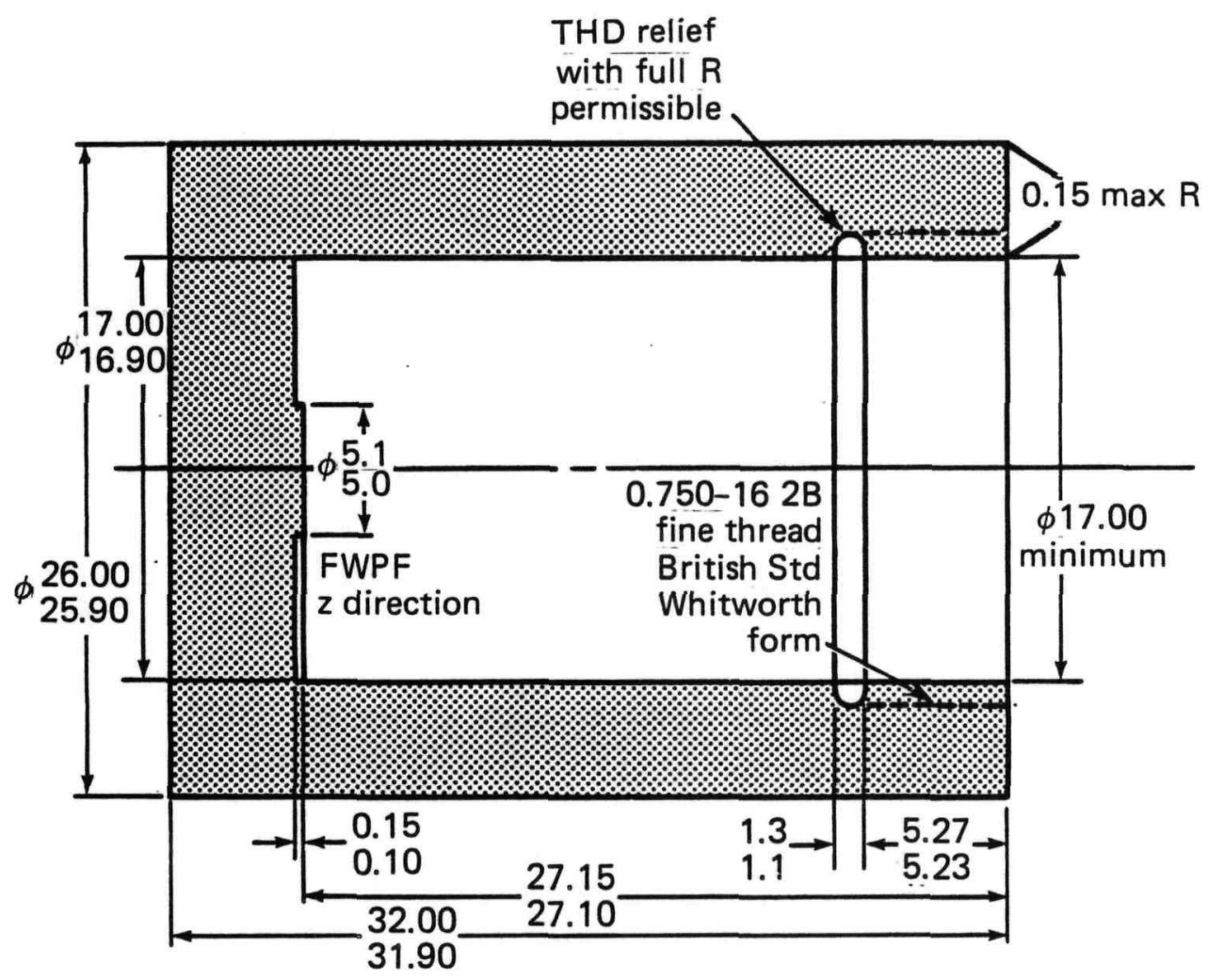

Matl-FWPF 30 graphite

Dimensions in mm: $\mathrm{W}=39.787 \mathrm{~g}$ (TYP) for LWRHU assembly

Fig. 2 LWRHU aeroshell body (reproduced from Ref. 2). 


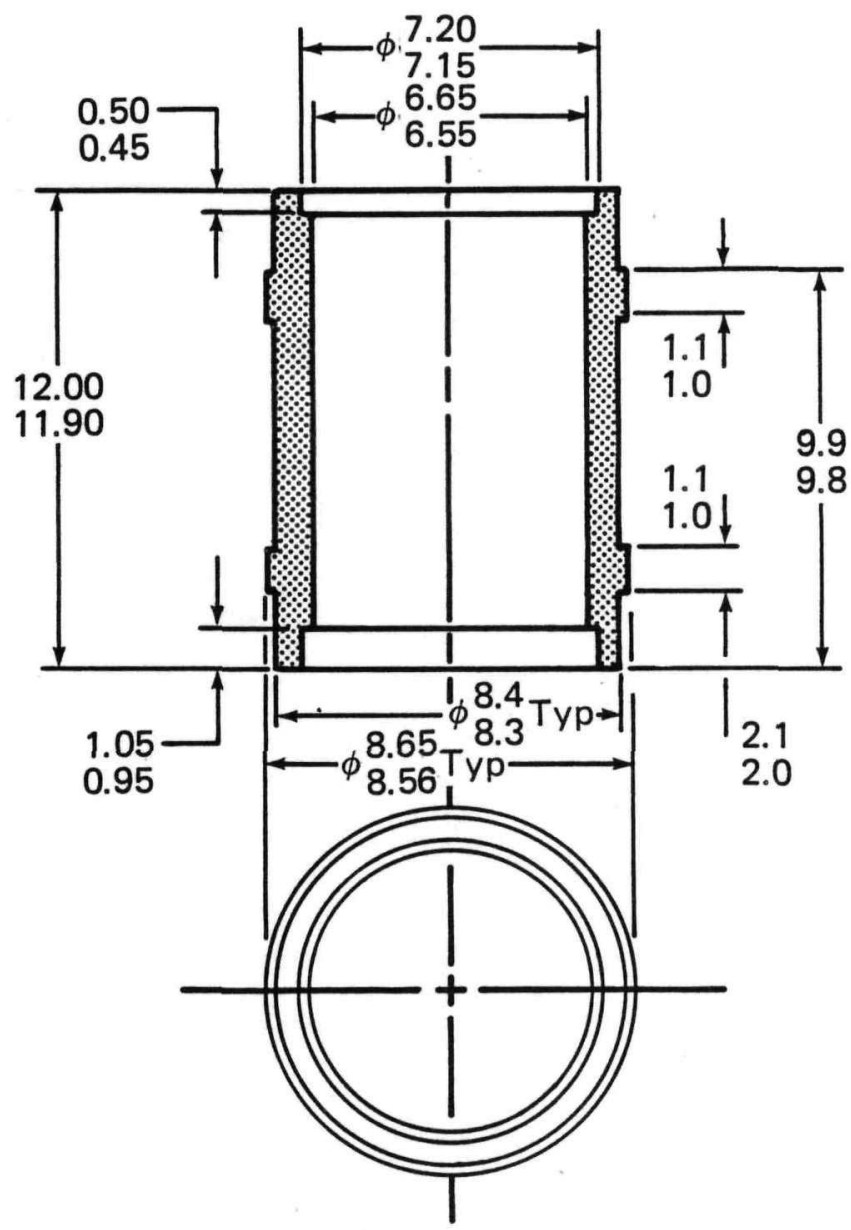

Dimensions in mm: $\mathrm{W}=8.366 \mathrm{~g}$ (TYP) for CLAD/FUEL assembly

Fig. 3 LWRHU clad body (reproduced from Ref. 2). 


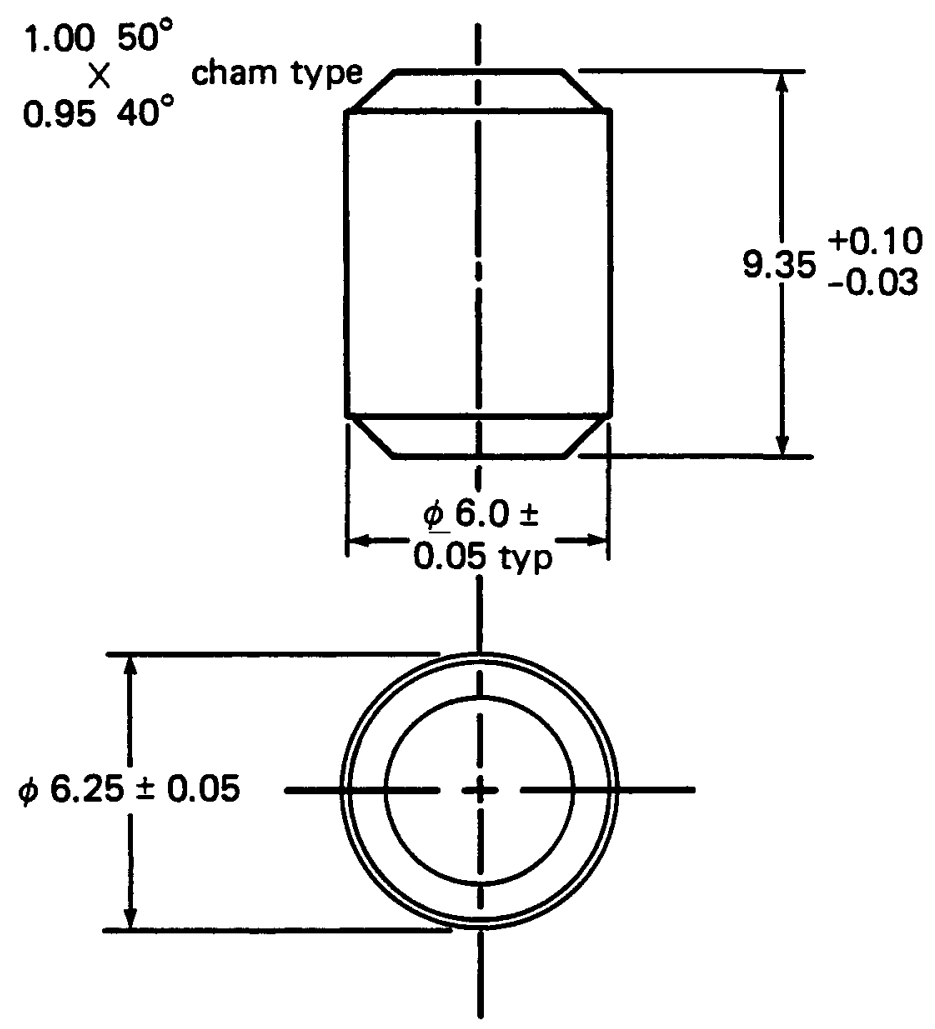

Dimensions in $\mathrm{mm}: \mathrm{W}=2.664 \mathrm{~g}$ (TYP)

Fig. 4 LWRHU fuel pellet (reproduced from Ref. 2). 


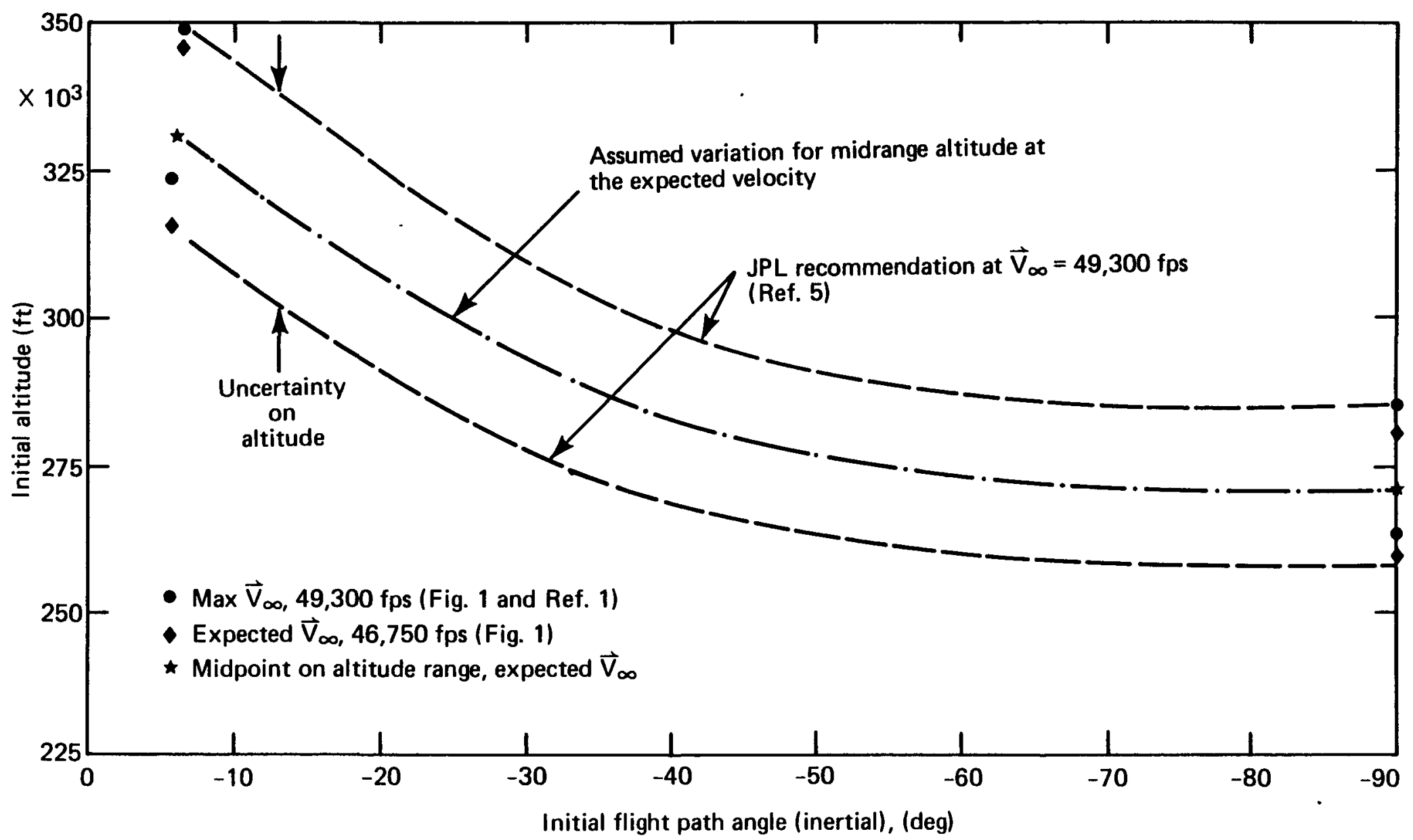

Fig. 5 Variation of initial altitude versus flight path angle for maximum and expected initial velocities for discrete GPHS module and LWRHU reentries; GalileoVEEGA mission profile (from Ref. 5). 


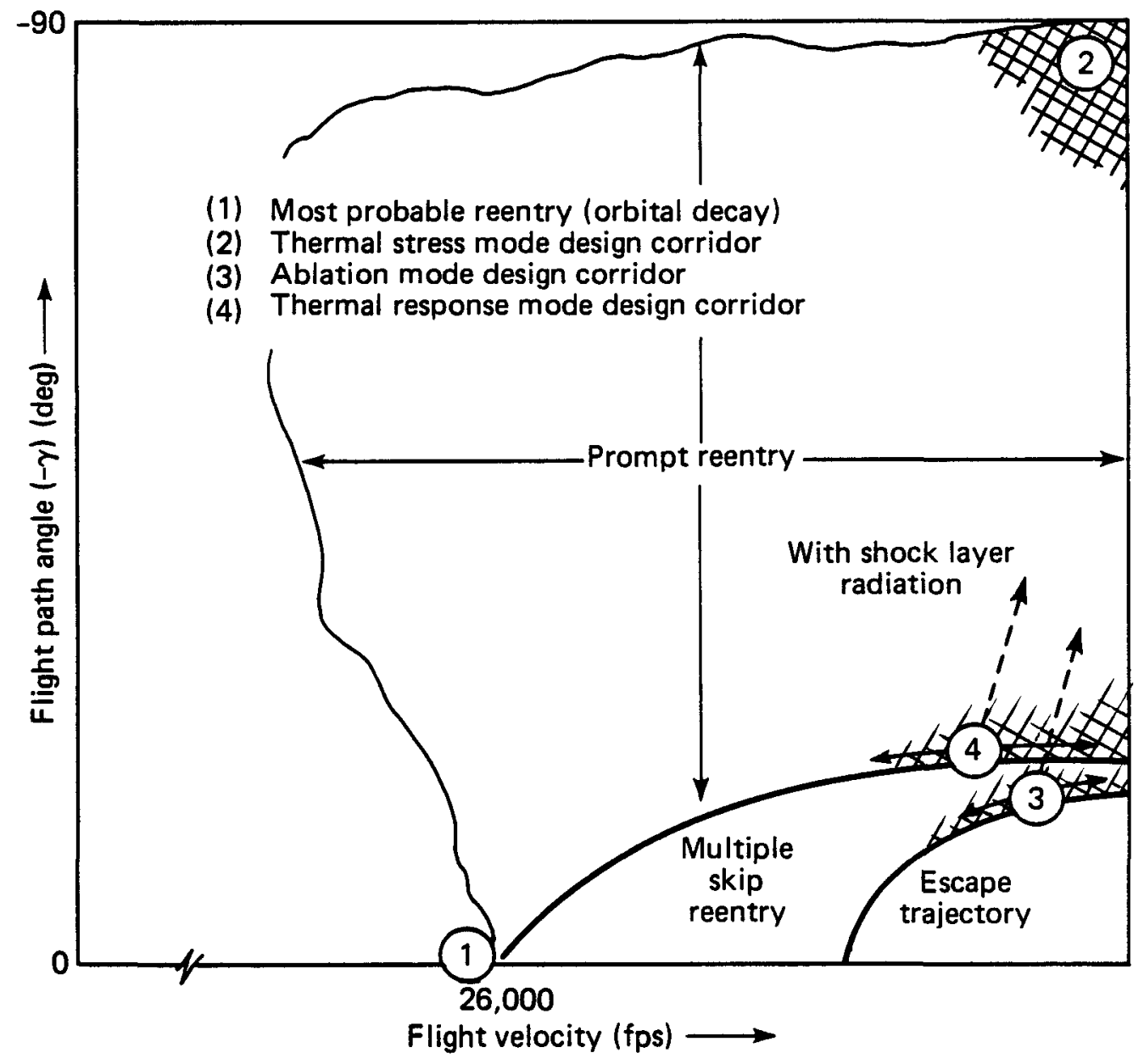

Fig. 6a Typical initial reentry velocity - flight path angle map; negligible shock layer radiation.
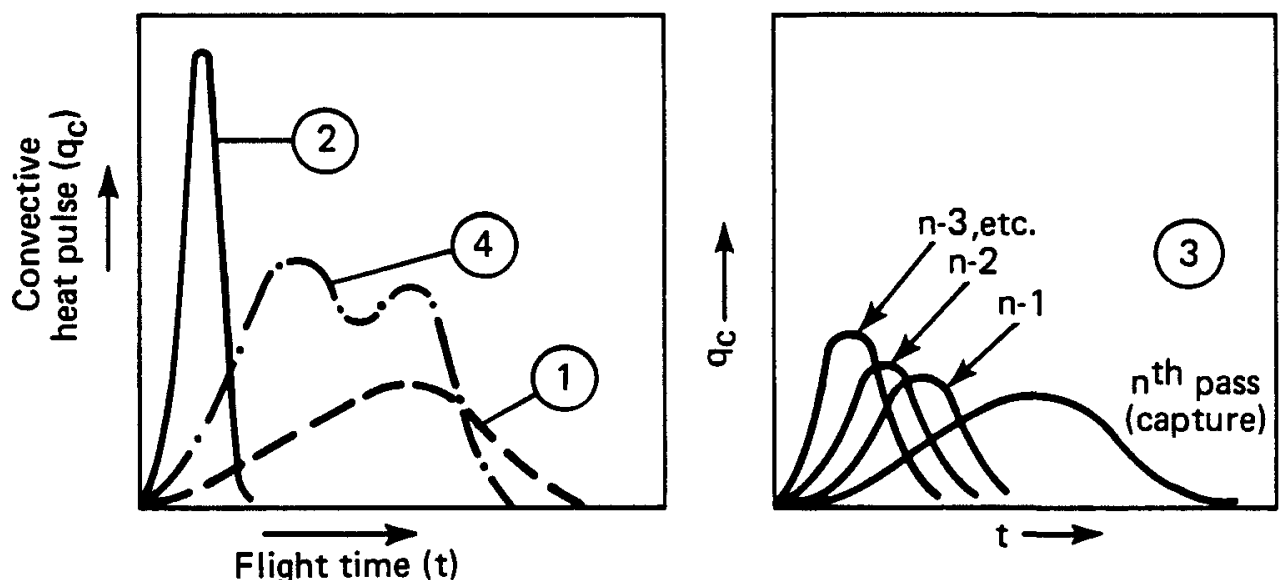

Fig. 6b Characteristic convective heat pulse for various heat source reentry design corridors (from Ref. 9). 


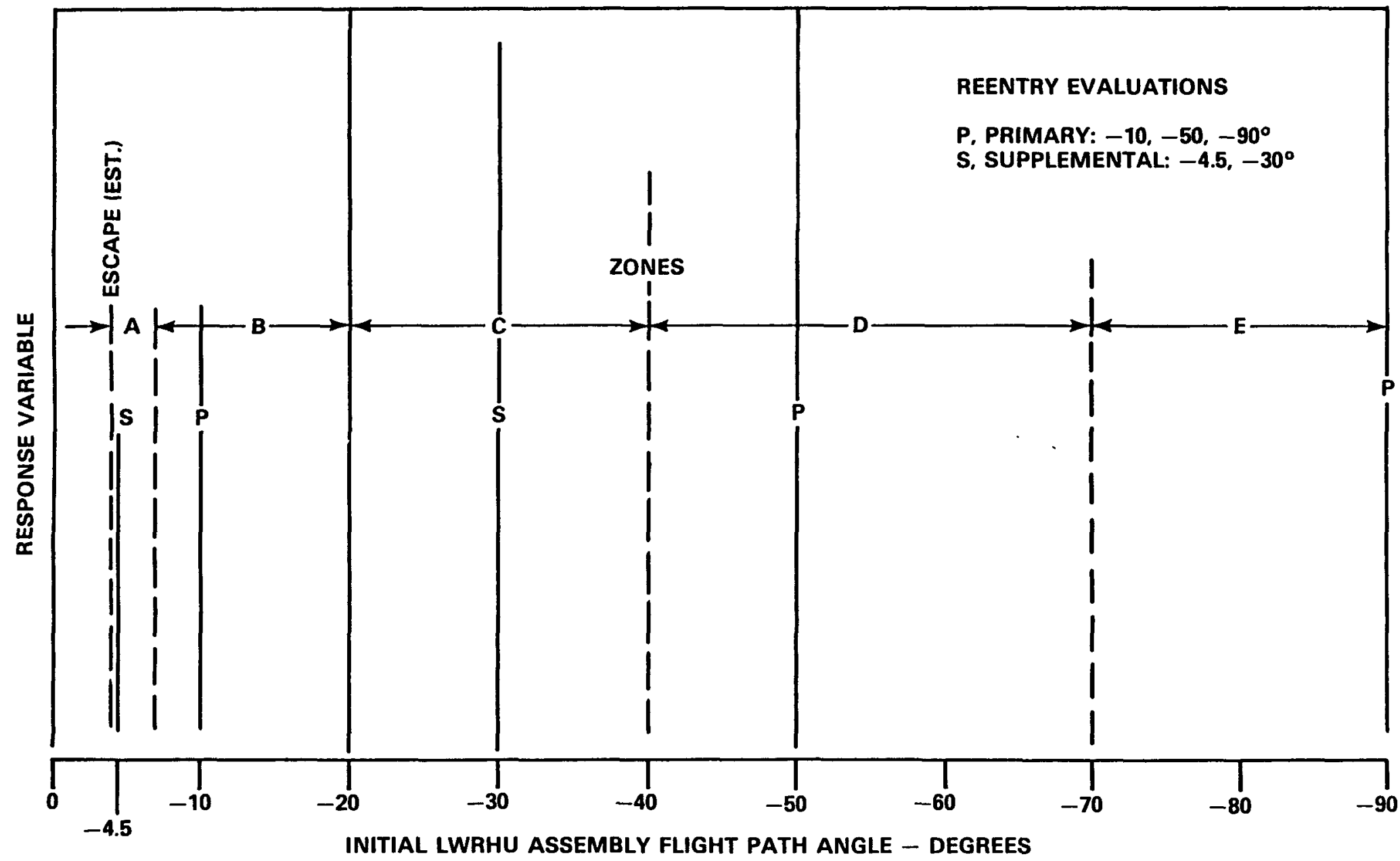

Fig. 7 Zonal evaluation of LWRHU reentry performance for Galileo/VEEGA; side-on stable LWRHU assembly reentry configuration. 
T 3 DOF trajectory analysis

S Supplemental reentry thermal evaluation

EB Estimated escape boundary

MPB Estimated multiple pass boundary

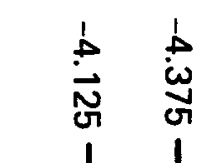

$1 \geq 1$

$\mid \begin{aligned} & 3 \\ & 1\end{aligned}$
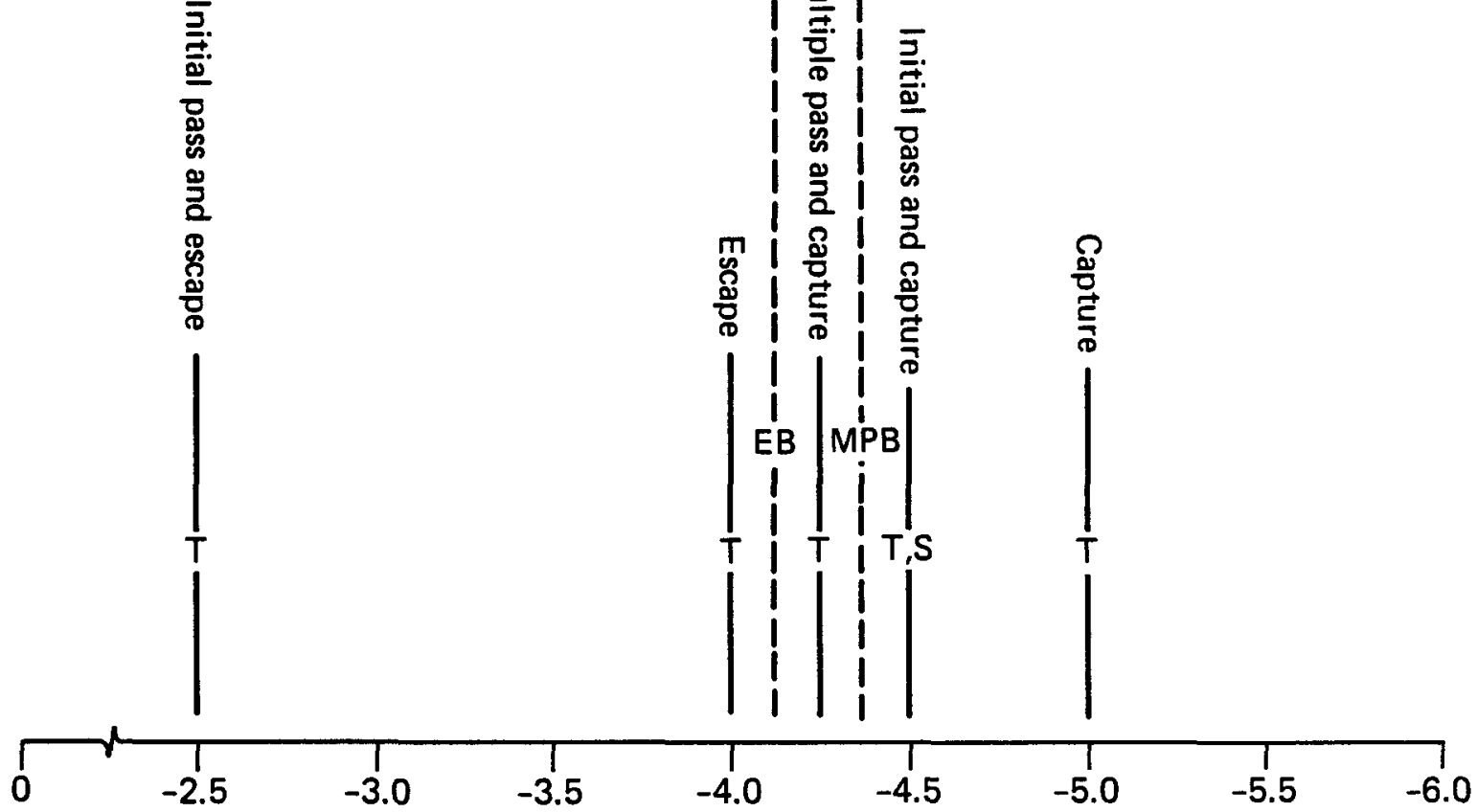

Initial flight path angle, inertial (deg)

Fig. 8 Enlarged view of the left hand extremum of the Galileo/VEEGA reentry map; side-on stable LWRHU assembly reentry configuration. 


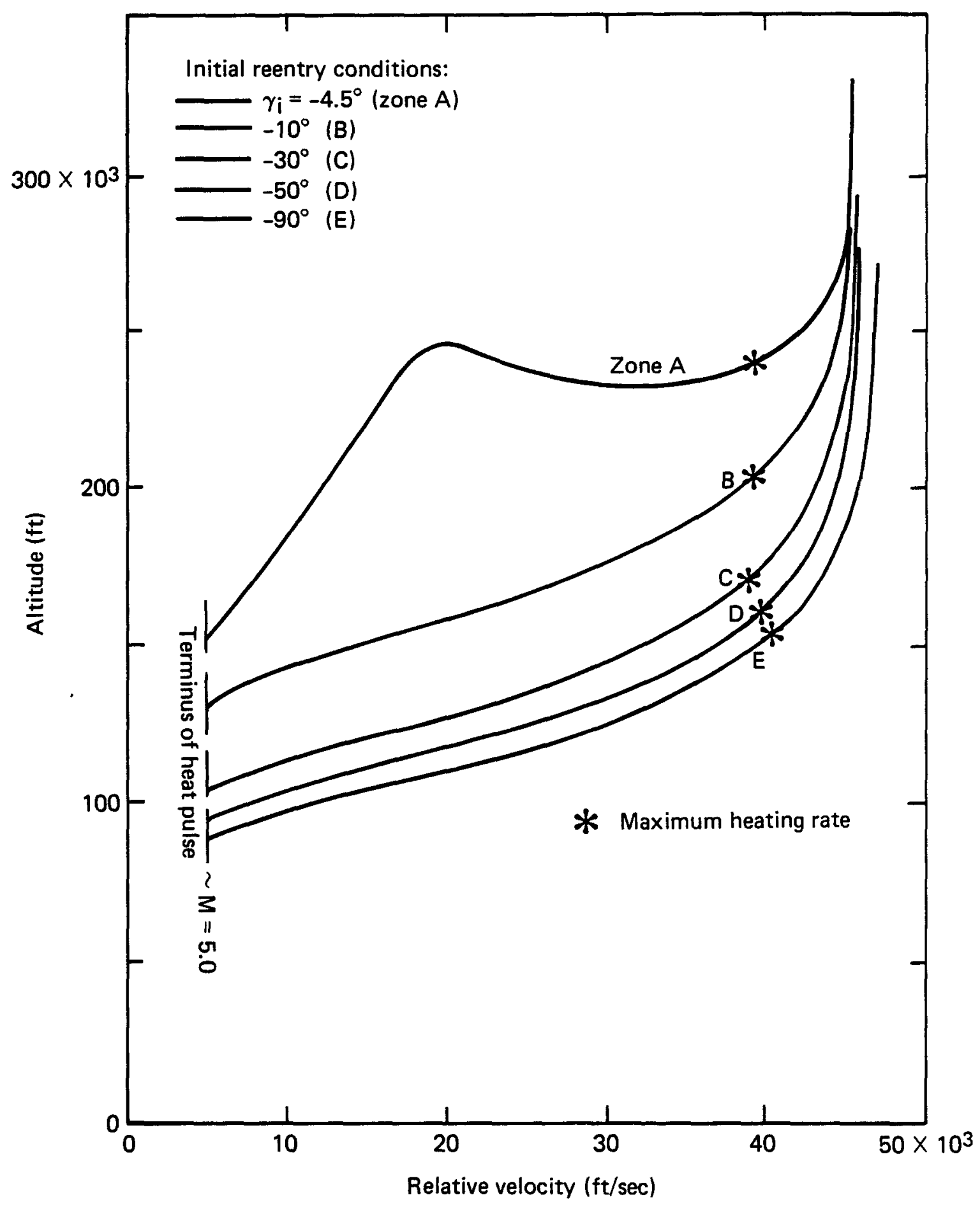

Fig. 9 Representative Galileo/VEEGA trajectory profiles for zones A through E; hypersonic flight period for side-on stable LWRHU reentry configuration. 


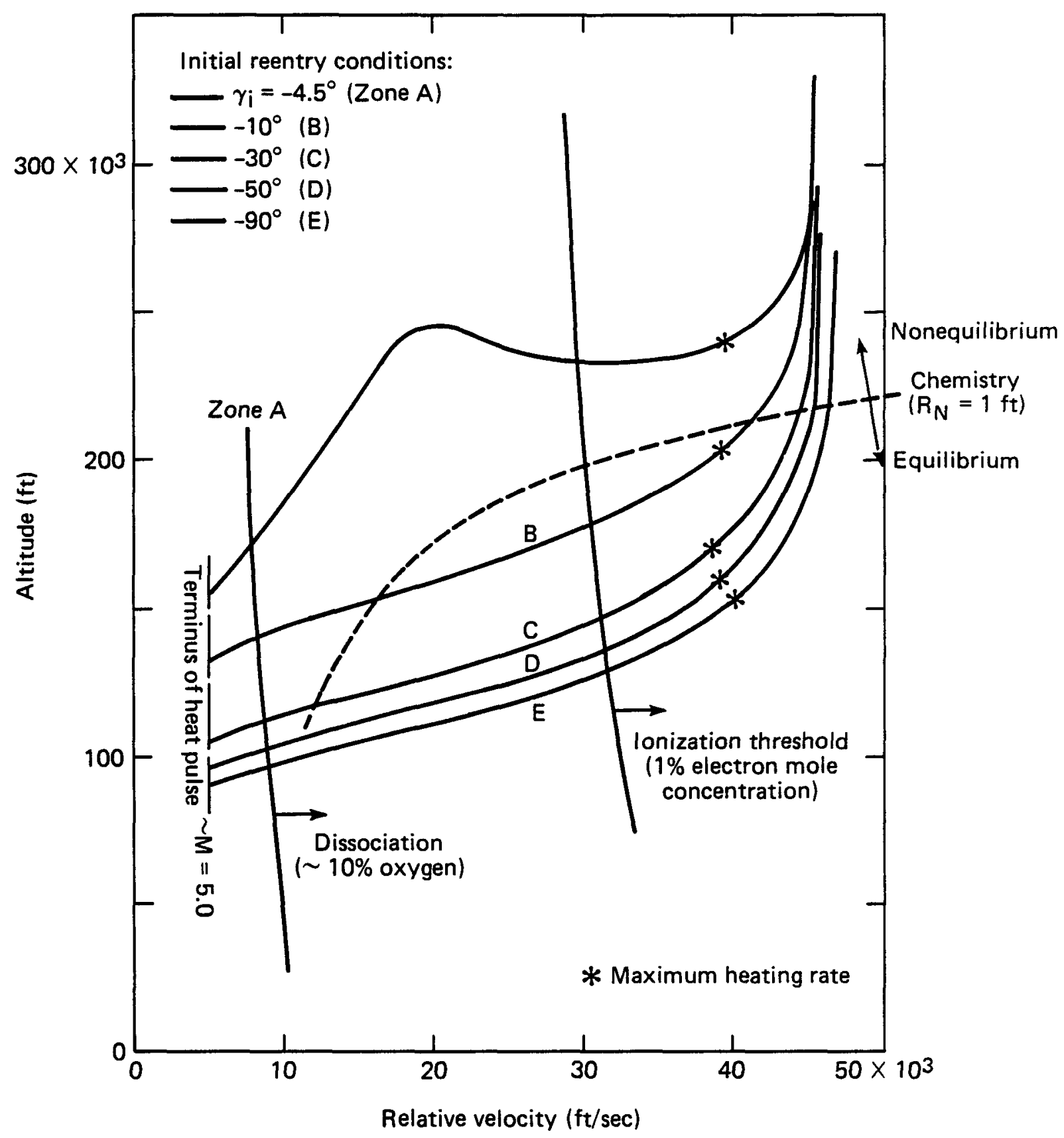

Fig. 10 Aerothermodynamic regimes for the Galileo/VEEGA zonal reentries; side-on stable LWRHU configuration. 


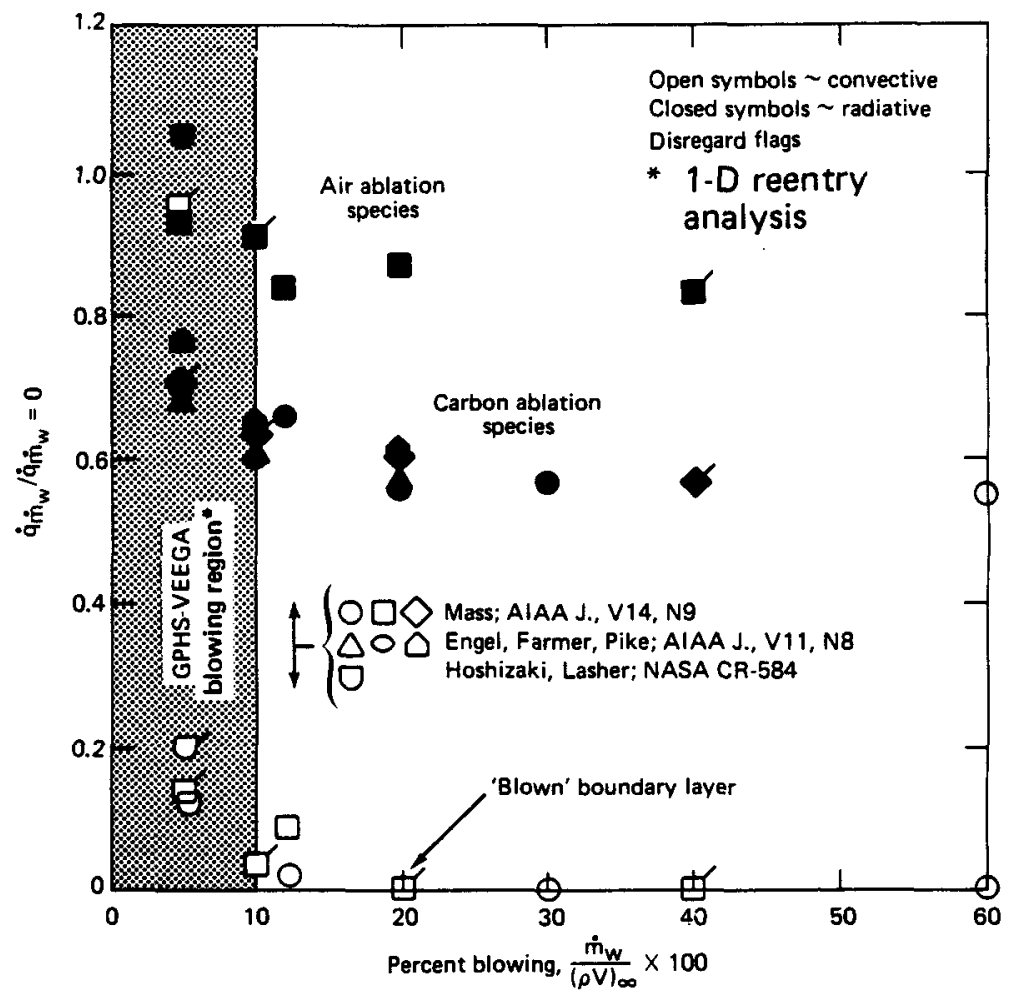

Fig. 11a The effect of mass transfer and chemical species on surface radiation and convective heat transfer for superorbital earth reentry conditions (Ref. 17).

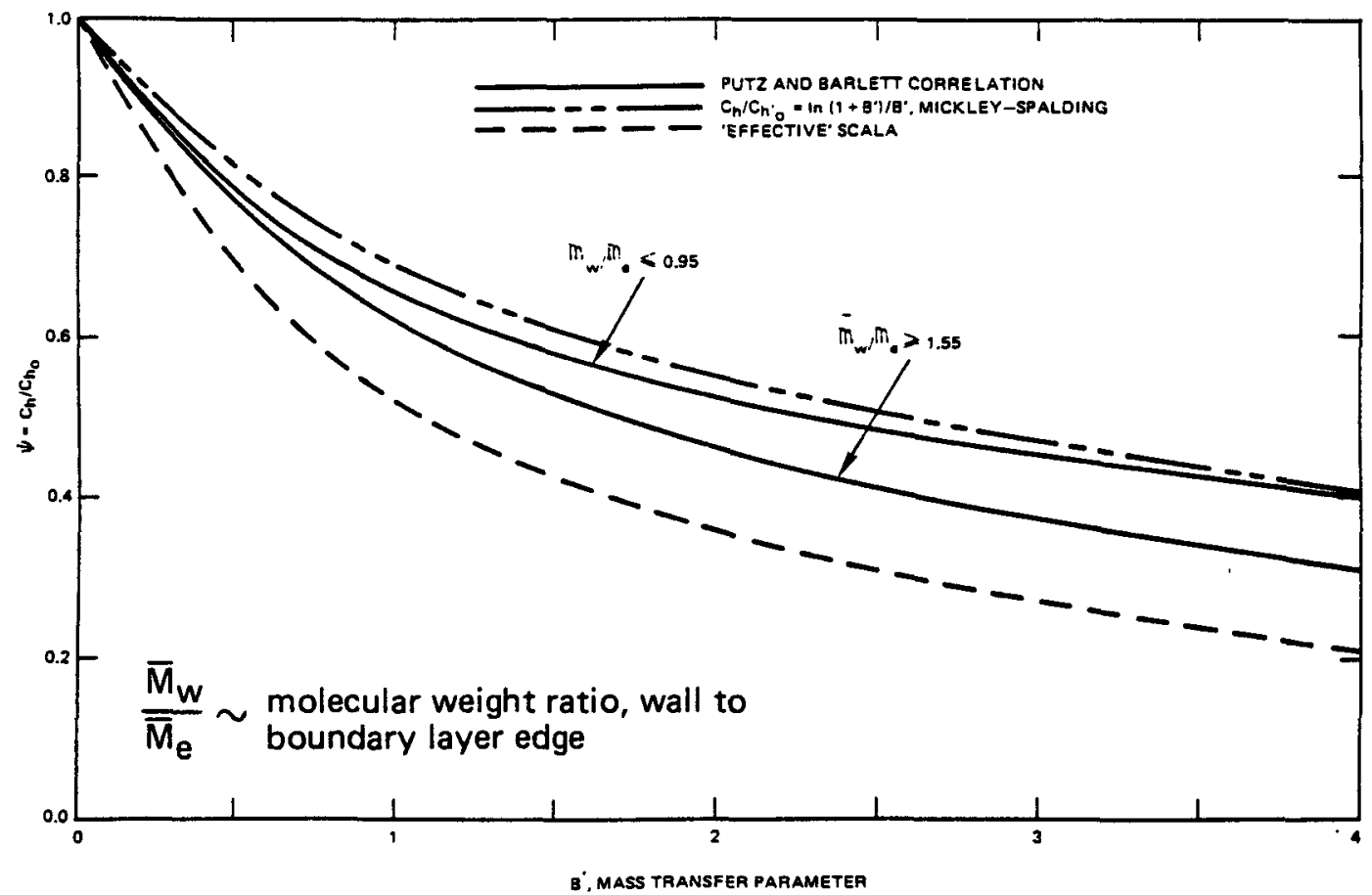

Fig. 11b Blowing-nonblowing heat transfer ratio $(\psi)$ as a function of the mass transfer parameter, $\beta^{\prime}$. (Ref. 43). 
THE JOHNS HOPKINS UNIVERSITY APPLIED PHYSICS LABORATORY

Laupel, Maryland

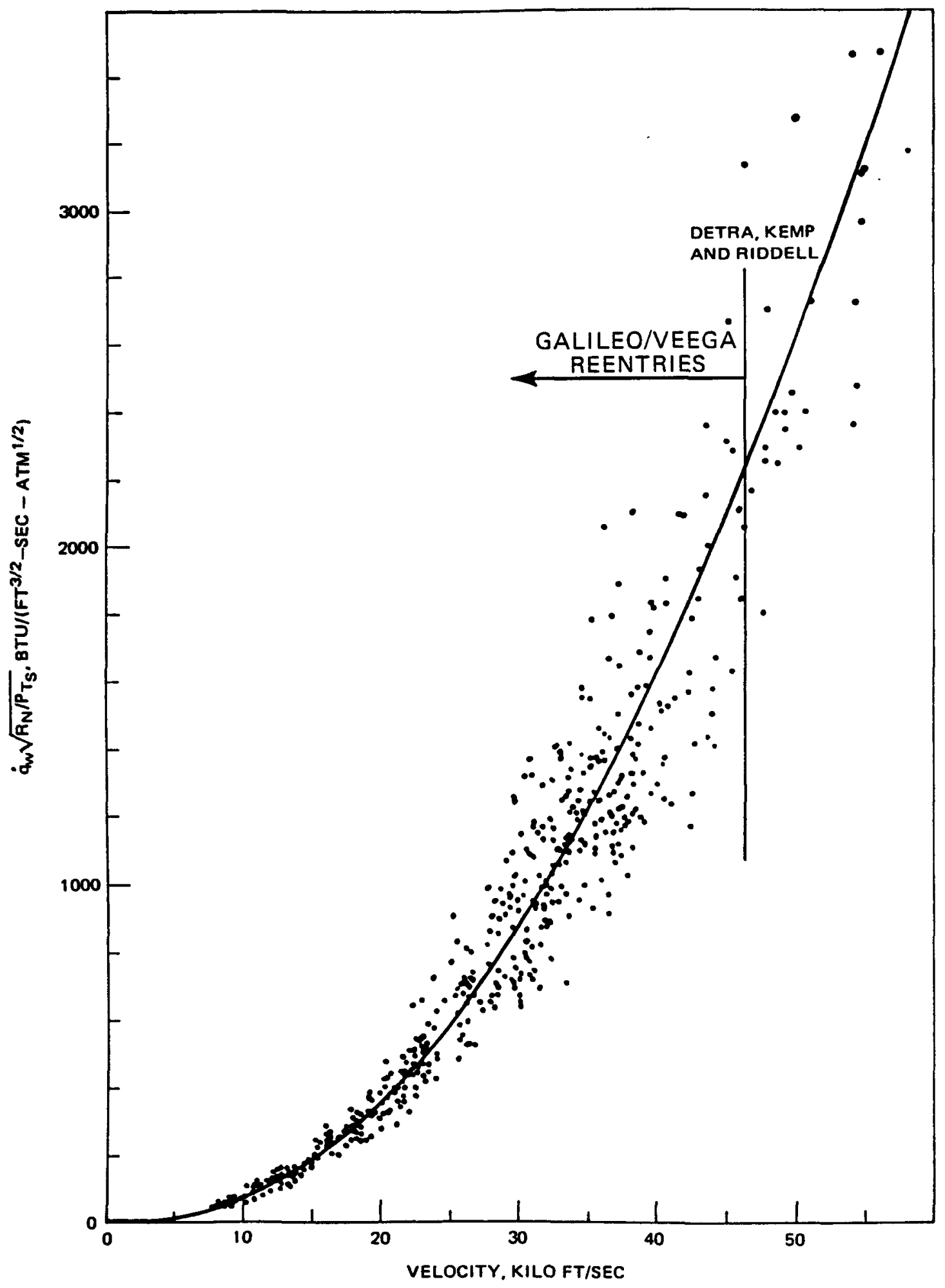

Fig. 12 Composite plot comparing experimental data with the Detra, Kemp and Riddell correlation (Ref. 14). 


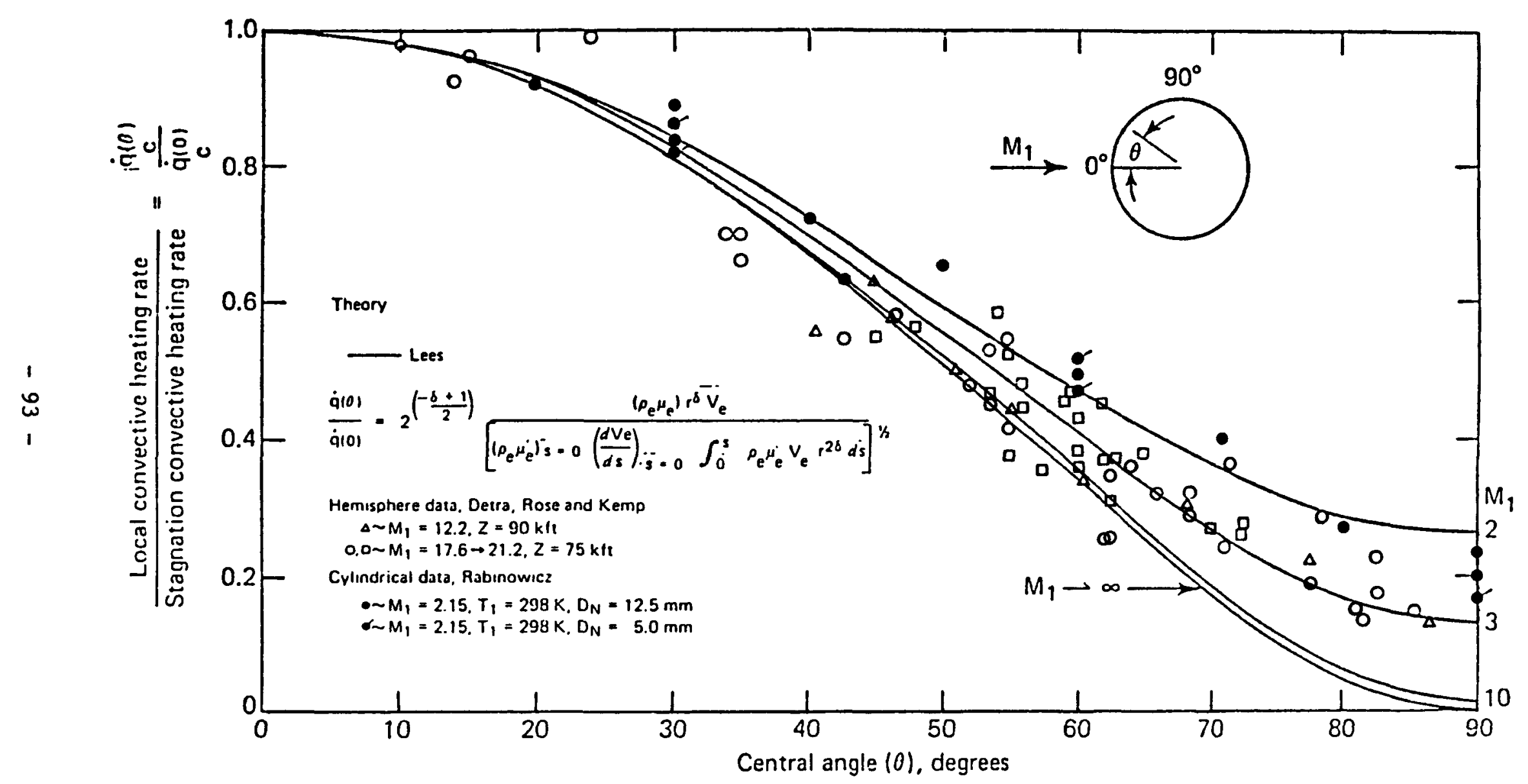

Fig. 13 Windward laminar boundary layer convective heating rate distribution. 


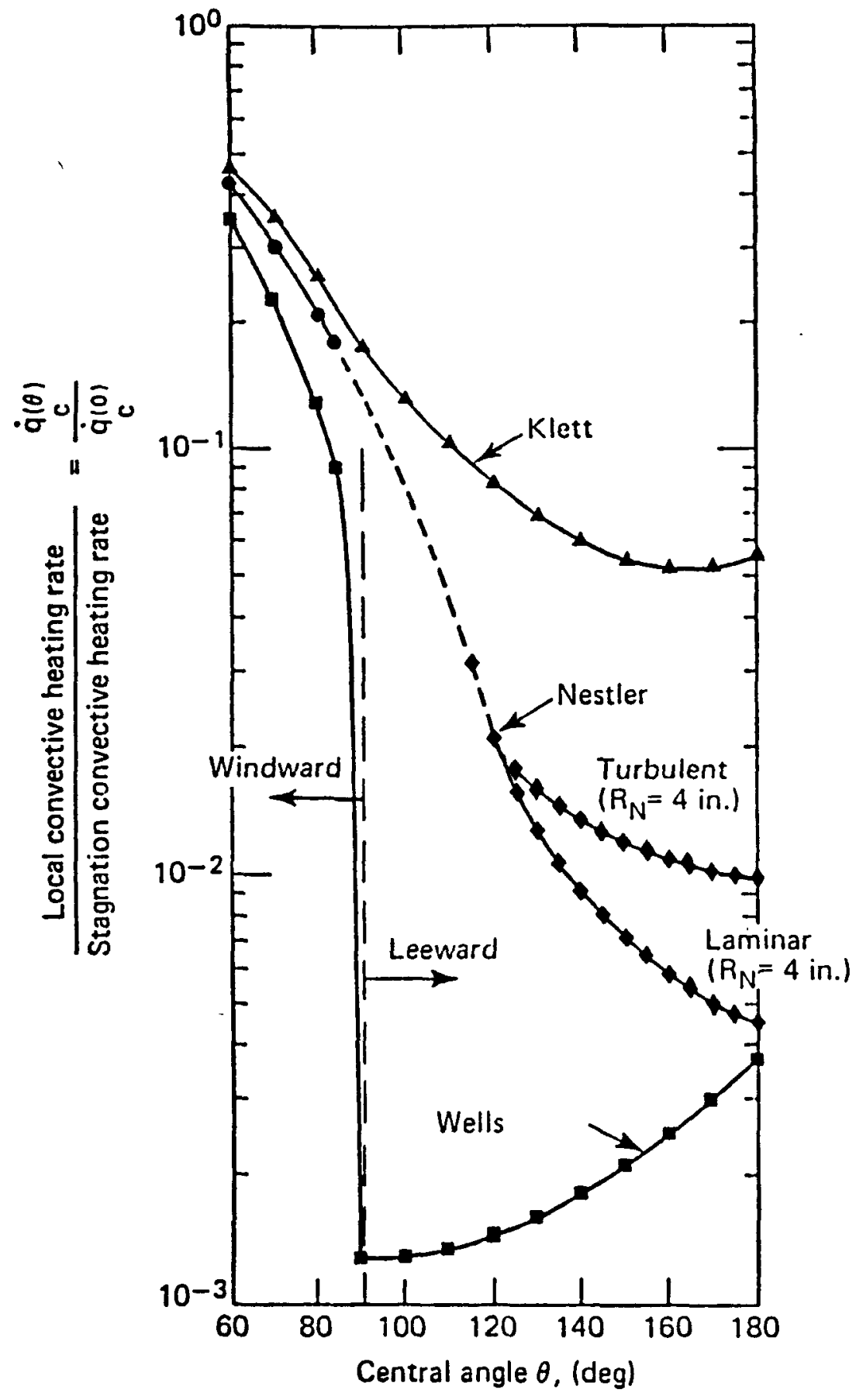

Fig. 14 Leeward convecting heating rate distributions around a sphere (Ref. 30). 


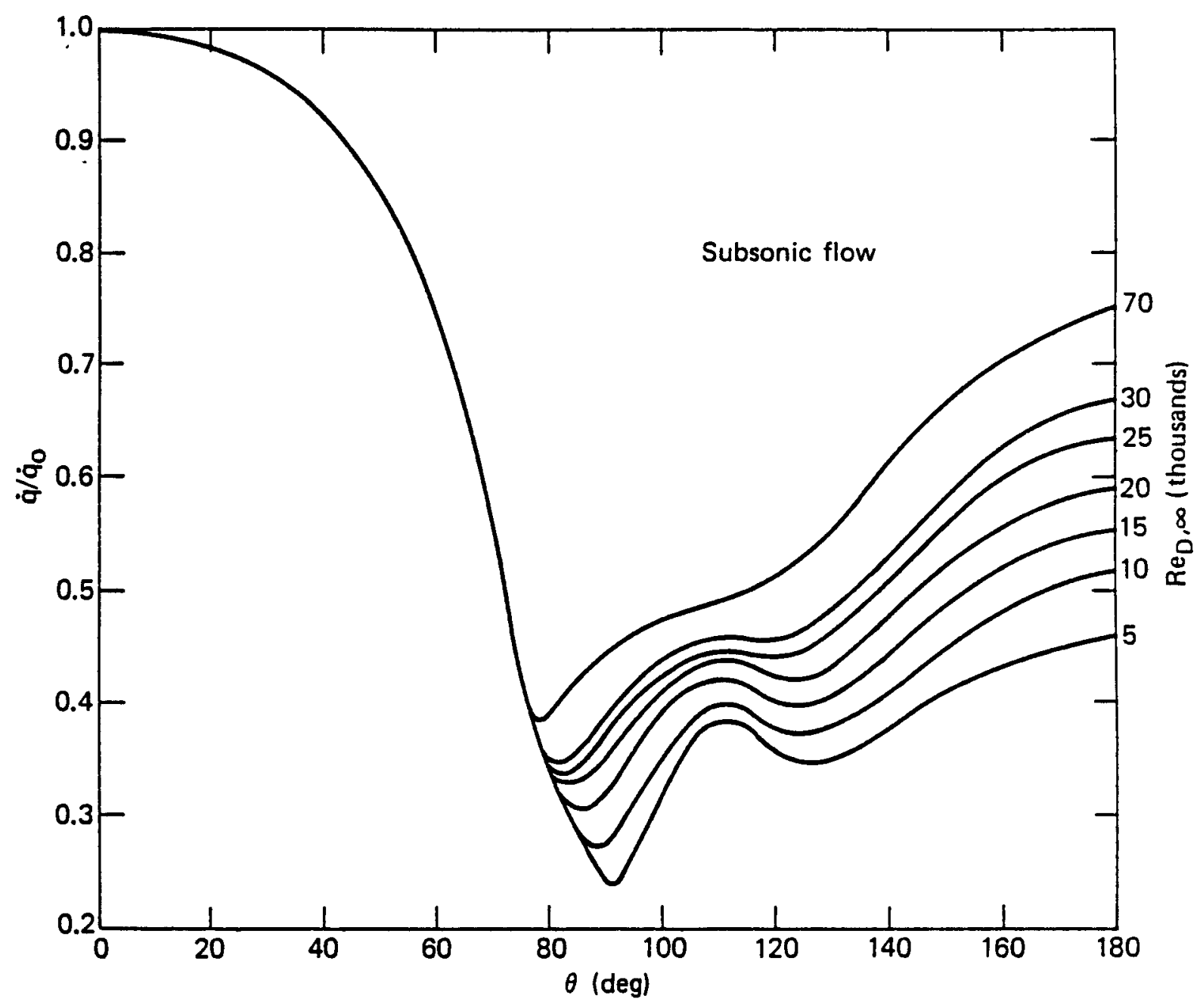

Fig. 15 Smoothed values of experimental heat transfer distribution around a circular cylinder (Ref. 32). 


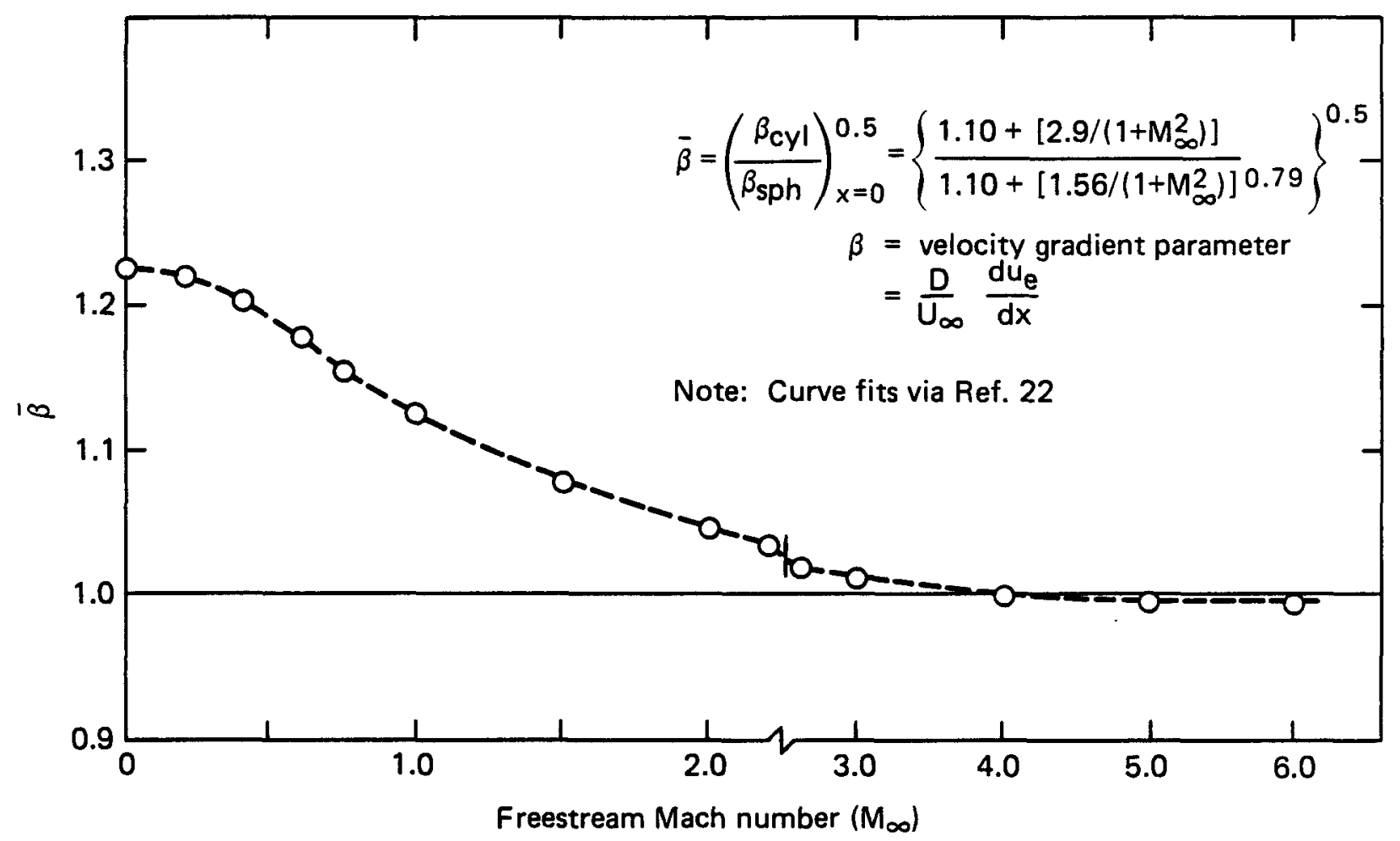

Fig. 16 Stagnation velocity gradient correction on the side-on LWRHU stagnation convective heating rate (without geometry scaling). 
- Capacitance (mass) nodes

- Surface (zero mass) nodes*

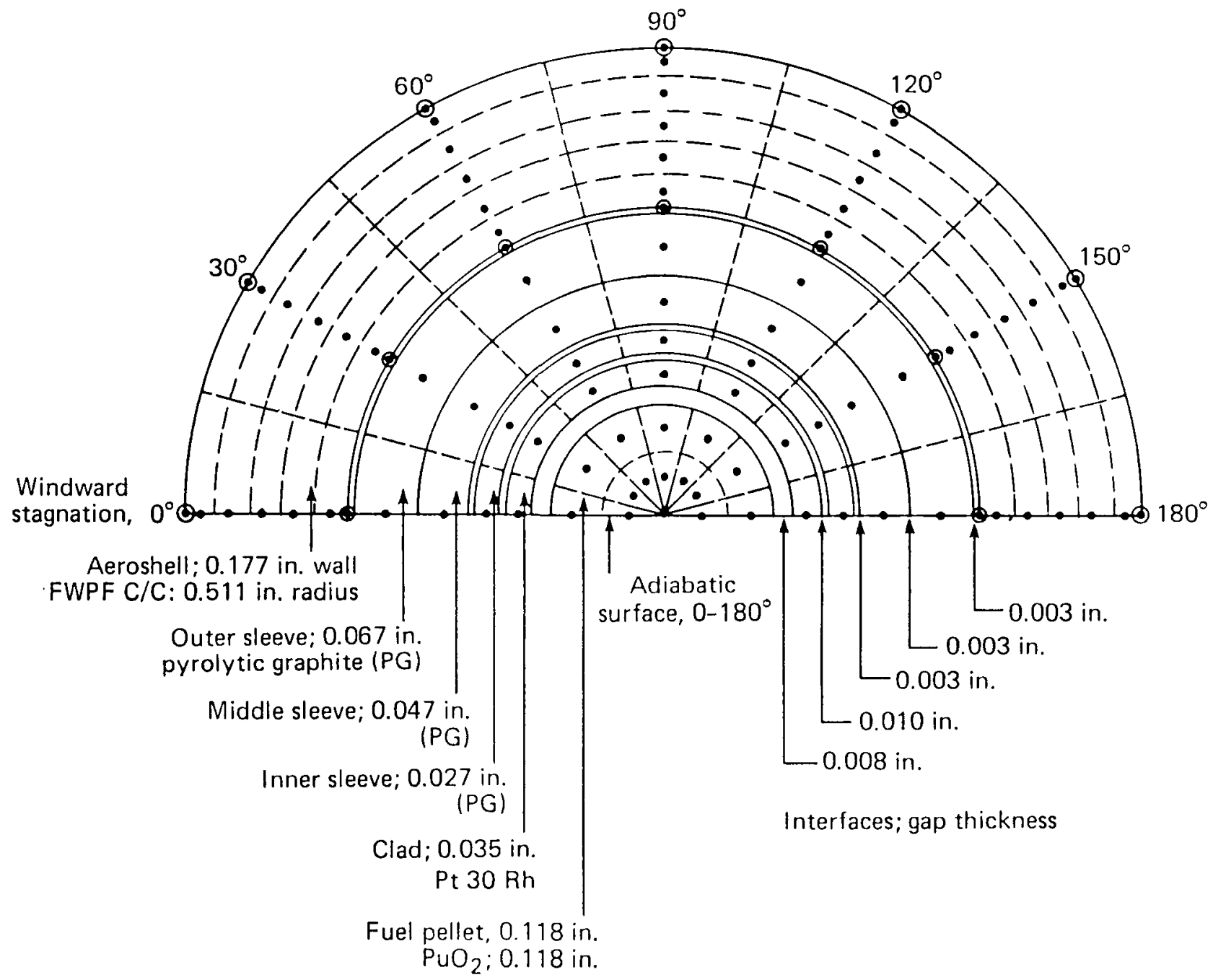

Component; wall thickness or radius material; outer radius

*Note: All internal surfaces assigned surface nodes; omitted for clarity purposes

Fig. 17a 2-D thermal model for side-on LWRHU assembly; mid-span cross section (not to scale). 


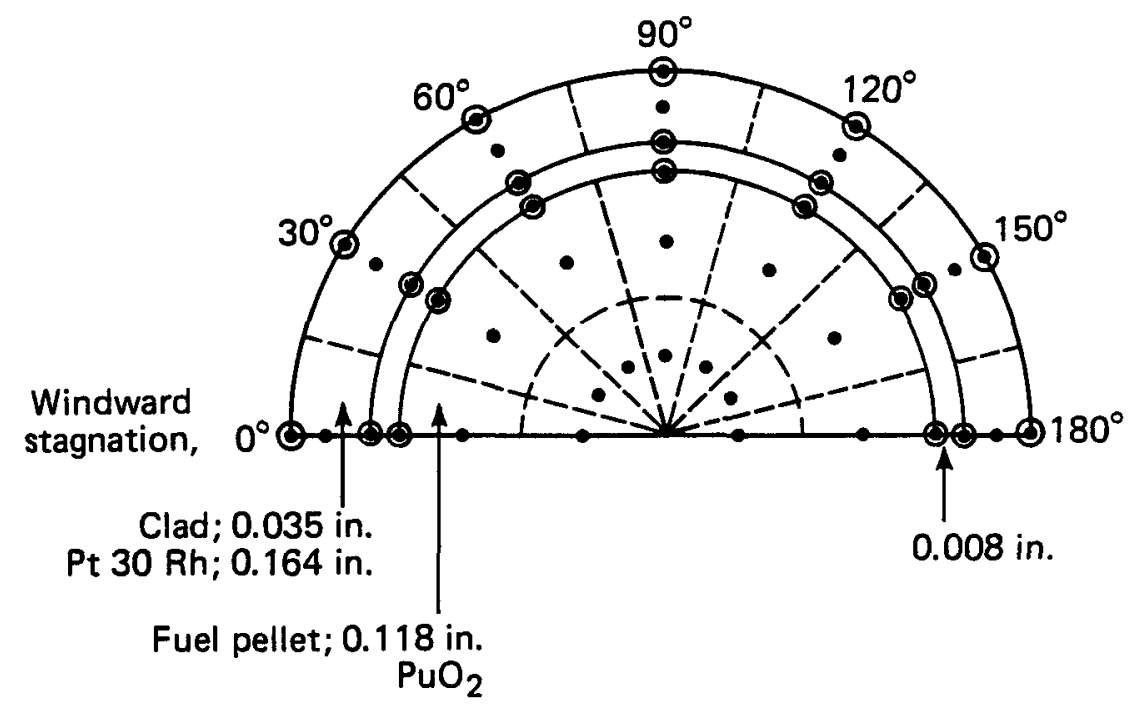

Note: model extracted directly from LWRHU assembly model; refer to Fig. 17a for details.

Fig. 17b 2-D thermal model for side-on clad/fuel assembly (not to scale).

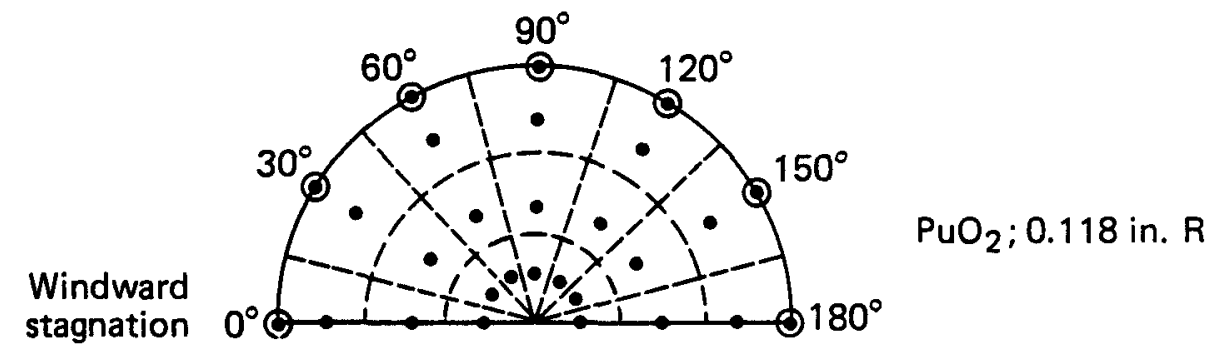

Fig. 17c 2-D thermal model for side-on fuel pellet (not to scale). 

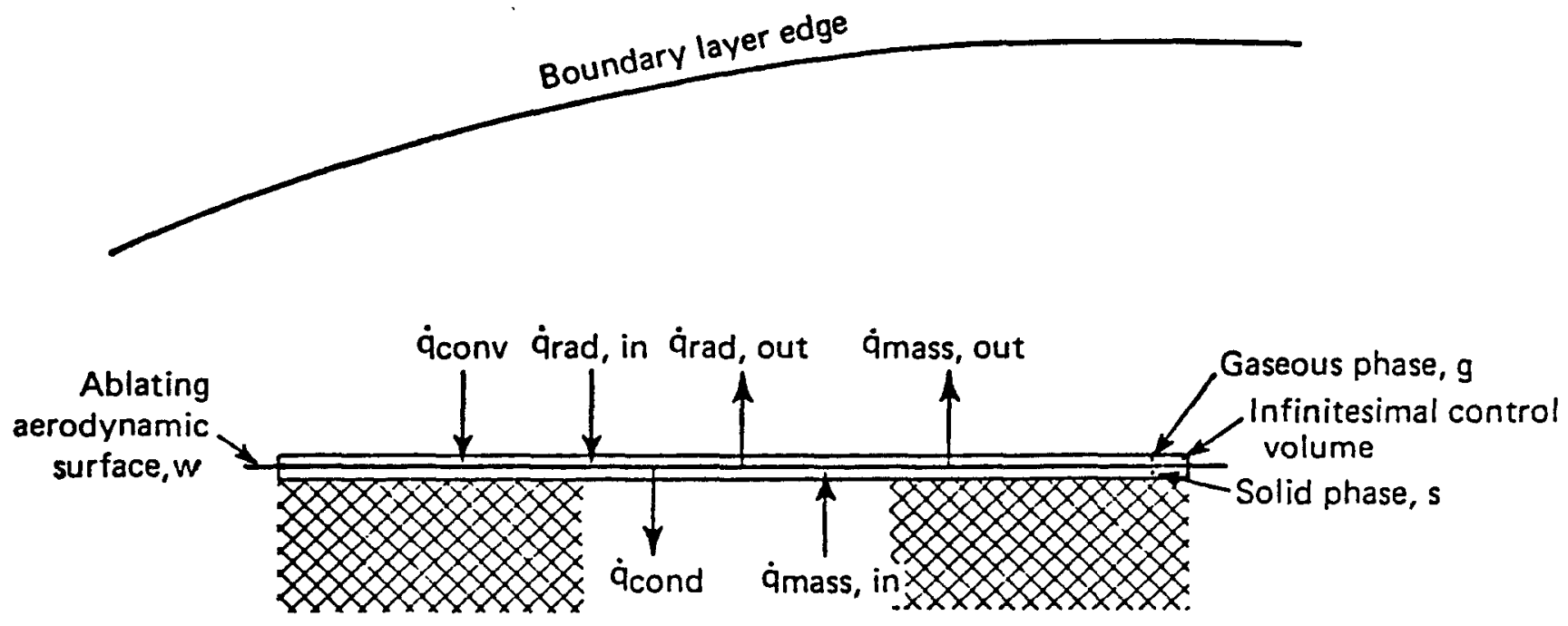

Boundary condition: $\sum_{i} \dot{q}_{i}=0$ where

- $\dot{\mathrm{q}}$ conv $\quad=$ Boundary layer convective transfer ccmprised of gaseous conduction transfer $\left(-k_{t_{g}} \partial T / \partial r\right)_{g, r}=w$ and diffusion transfer $\rho \sum_{i} D_{i j} H_{i}\left(\partial k_{i} / \partial r\right) r=w$

- $\dot{q}_{\text {rad, in }}=$ Shock layer radiative transfer

- $\dot{q}_{\text {rad, out }}=$ Surface emissive transfer, $\sigma \epsilon_{h} T_{W}^{4}$

- $\dot{q}_{\text {cond }} \quad=$ Solid conduction (Fourier) transfer, $-k_{t_{s}}(\partial T / \partial r)_{s, r}=w$

- $\dot{q}_{\text {mass, in }}=$ Mass flux transfer-solid phase, $\dot{m} H_{w_{s}}$

- $\dot{q}_{\text {mass, }}$ out $=$ Mass flux transfer-gaseous phase, $\left.\dot{m} H_{w_{g}}\right\}$

Energy transfer due to thermochemical ablation

Fig. 18 Surface energy balance - reentry thermal analysis. 
THE JOHNS HOPKINS UNIVERSITY APPLIED PHYSICS LABORATORY

LAUREL. MAAYLANO

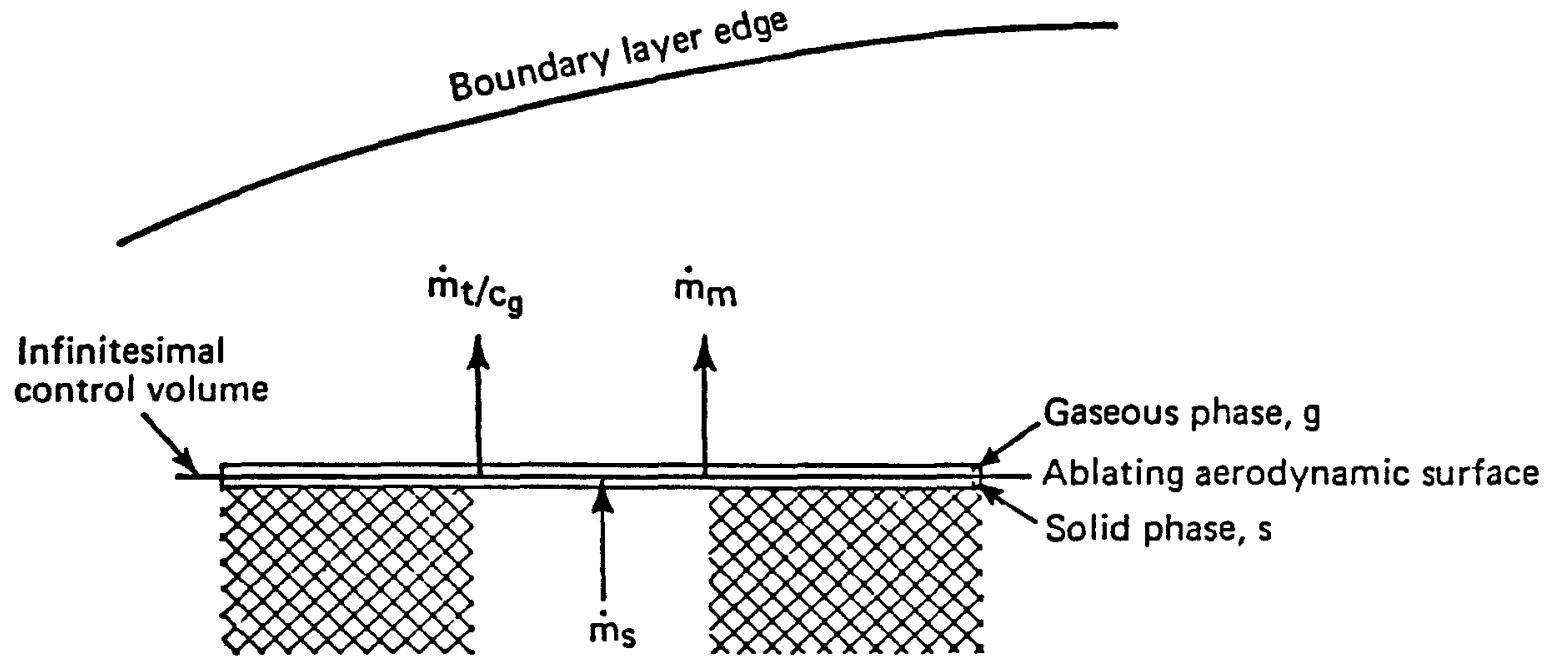

Boundary condition: $\sum_{i} \dot{m}_{i}=0$

- $\dot{\mathrm{m}}_{\mathrm{s}} \sim$ Mass flux due to ablation, solid phase

- $\dot{\mathrm{m}}_{\mathrm{t}} / \mathrm{c}_{\mathrm{g}} \sim$ Mass flux due to thermochemical ablation, gaseous phase

- $\dot{\mathrm{m}}_{\mathrm{m}} \sim$ Mass flux due to mechanical ablation, solid phase

Fig. 19 Surface mass balance - reentry thermal analysis. 


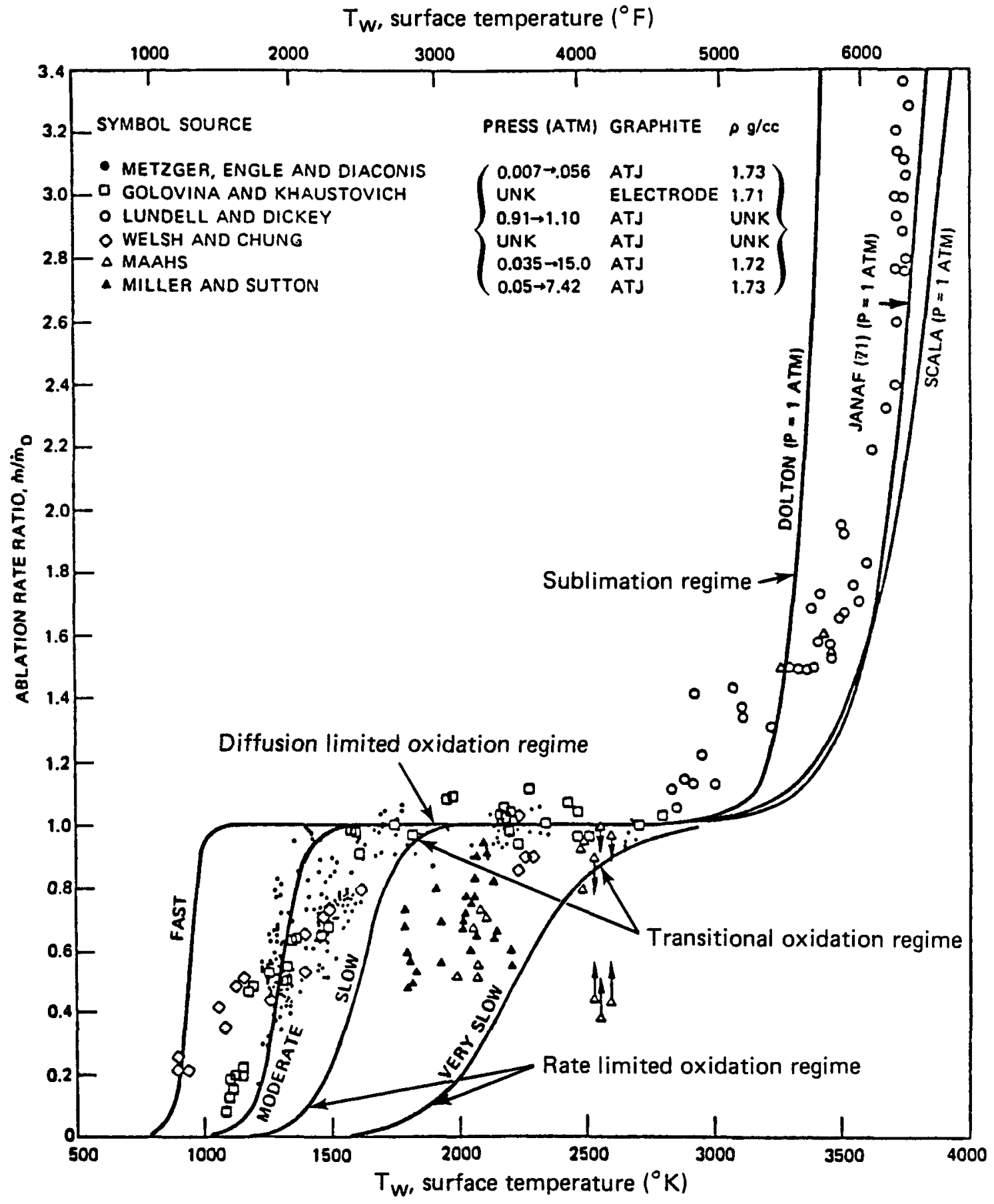

Fig. 20 Thermochemical ablation regimes for graphite. 


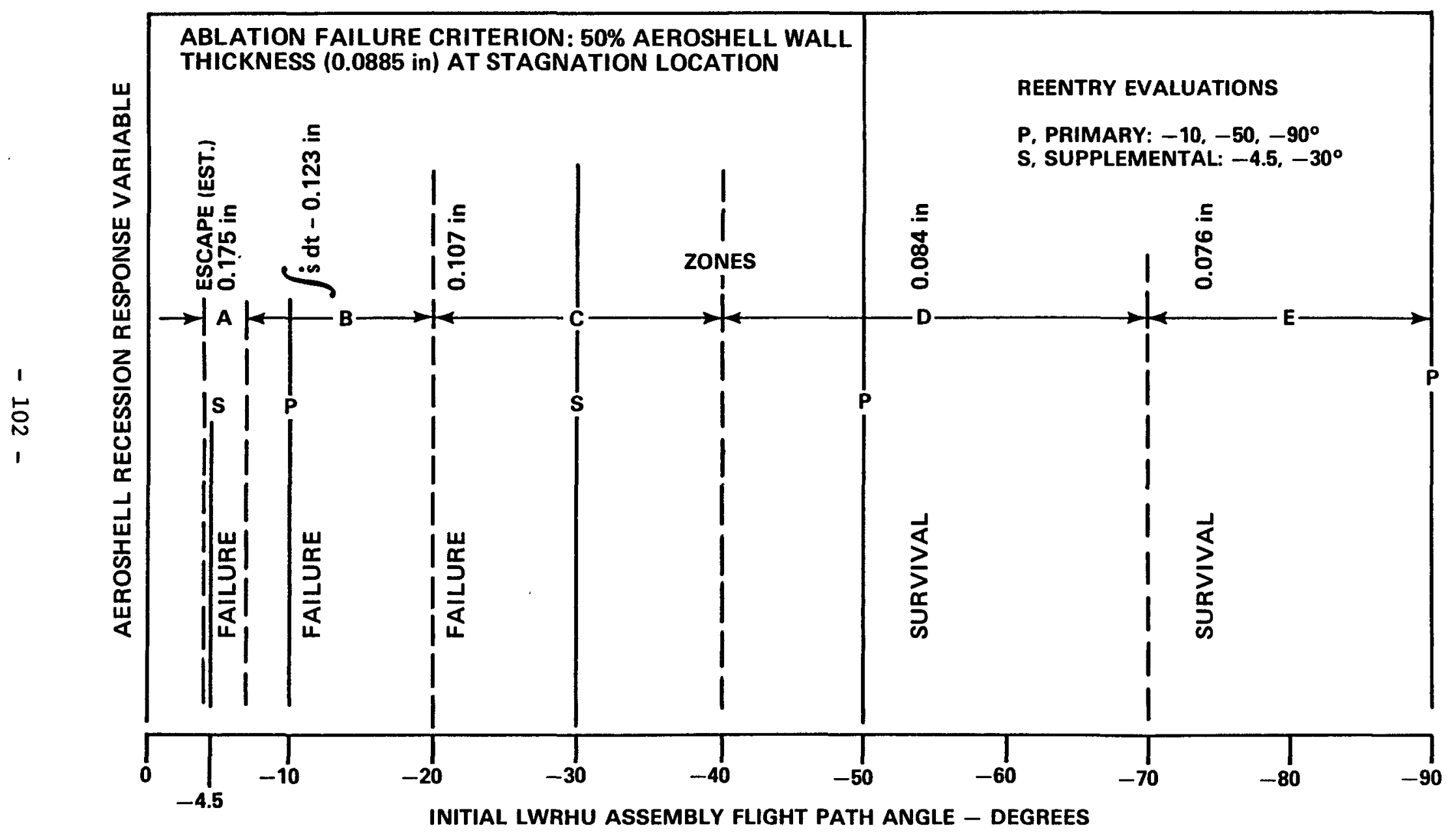

Fig. 21 Aeroshell total stagnation recession predictions; side-on stable LWRHU assembly reentry configuration. 


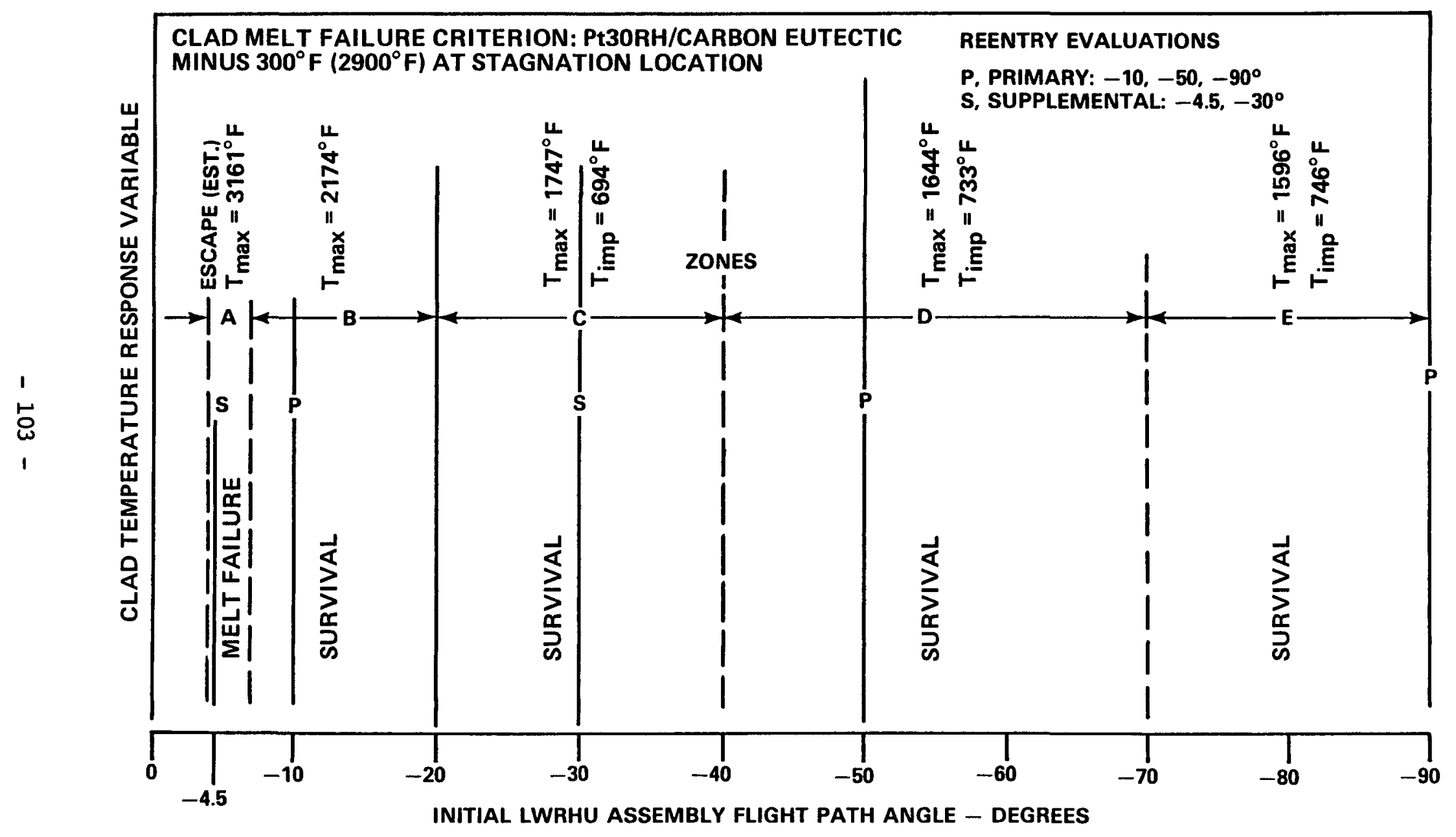

Fig. 22 Clad stagnation maximum and impact temperatures (assuming no prior ablation or thermal stress failure events); side-on stable LWRHU assembly reentry configuration. 


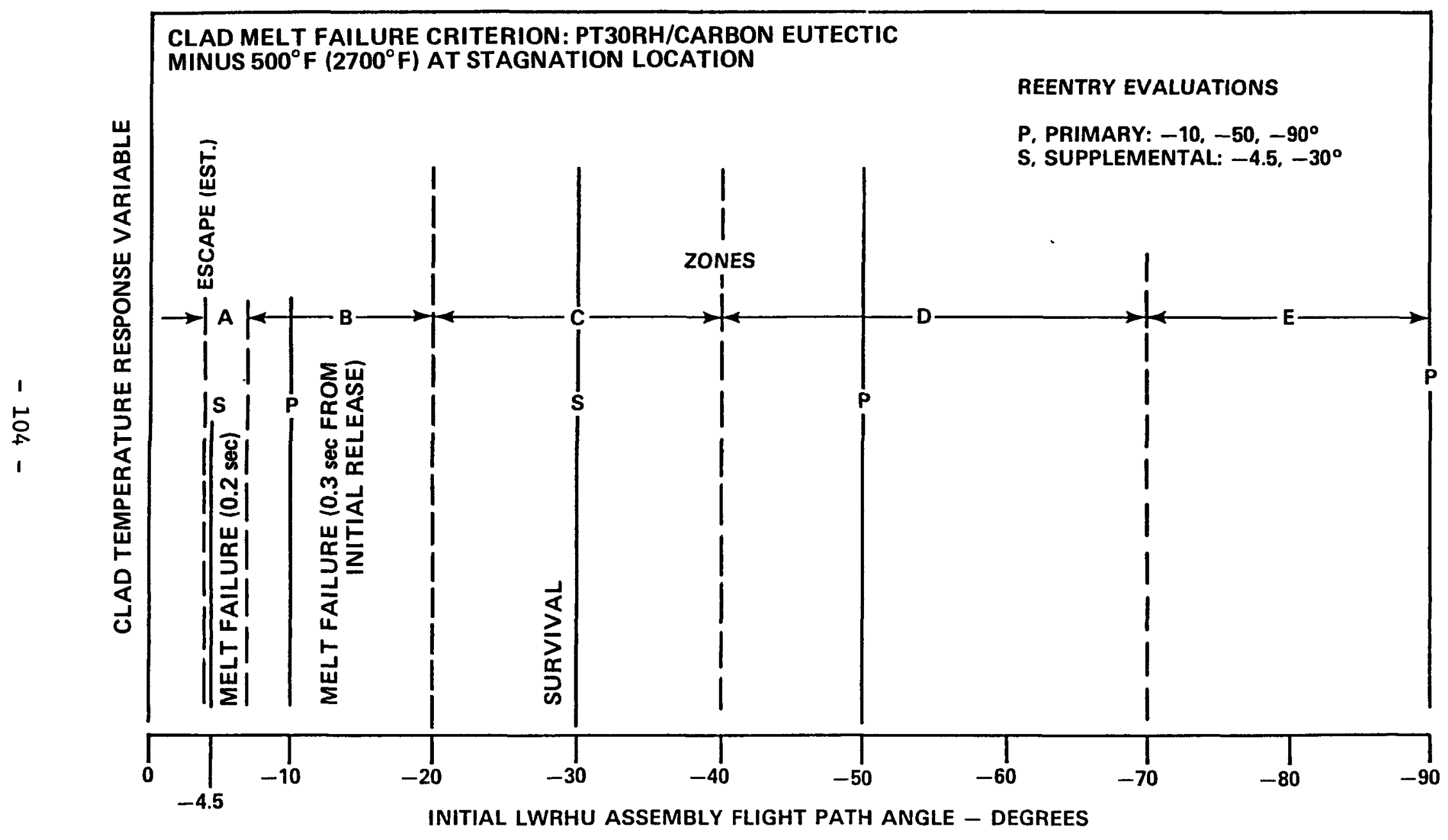

Fig. 23 Clad thermal response behavior subsequent to aeroshell failure (Fig. 21) and release; side-on stable clad/fuel assembly reentry configuration. 


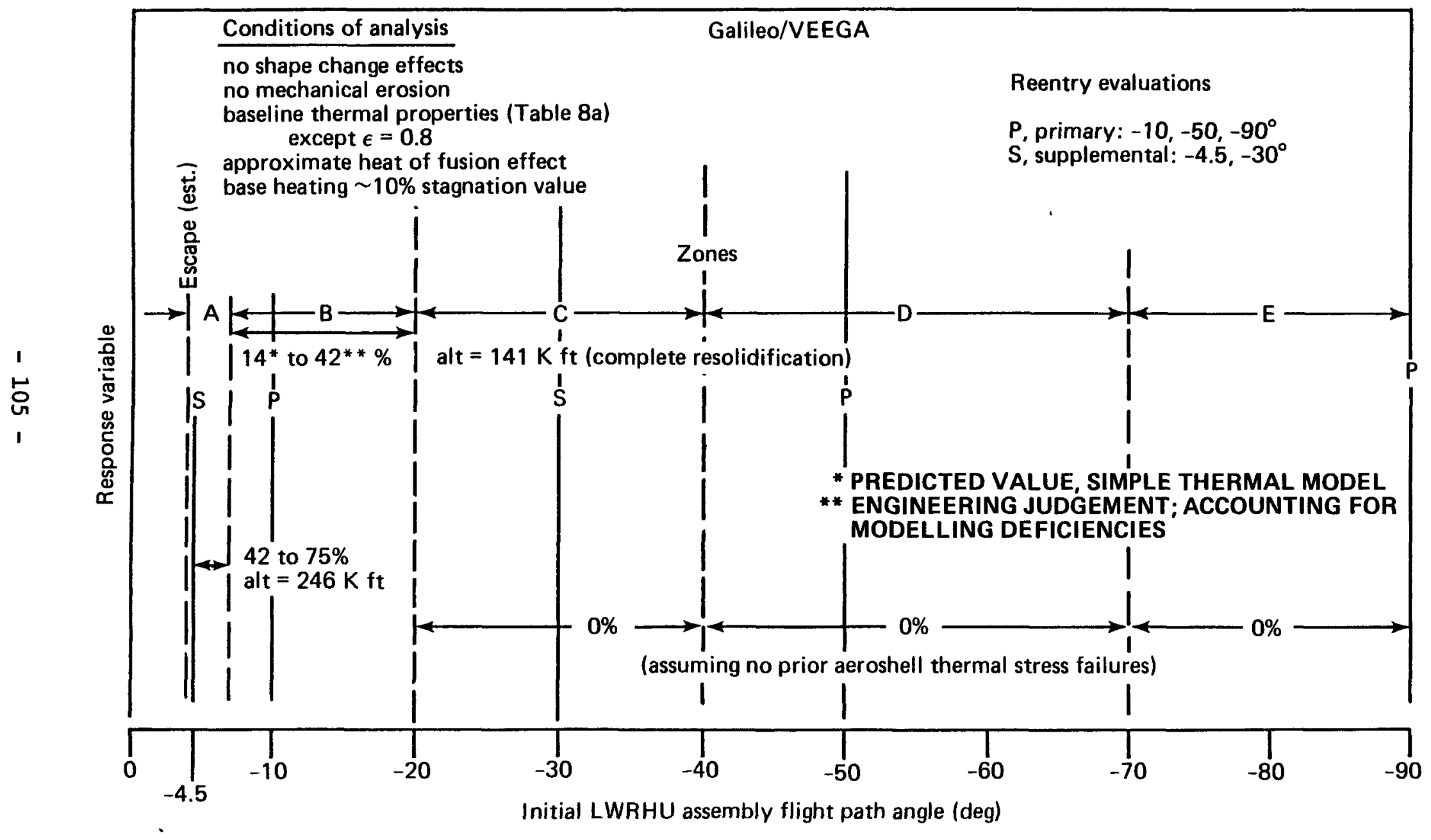

Fig. 24 Estimate of mass fraction of fuel melt resulting from prior sequential failure of the LWRHU; side-on stable bare fuel pellet reentry configuration. 


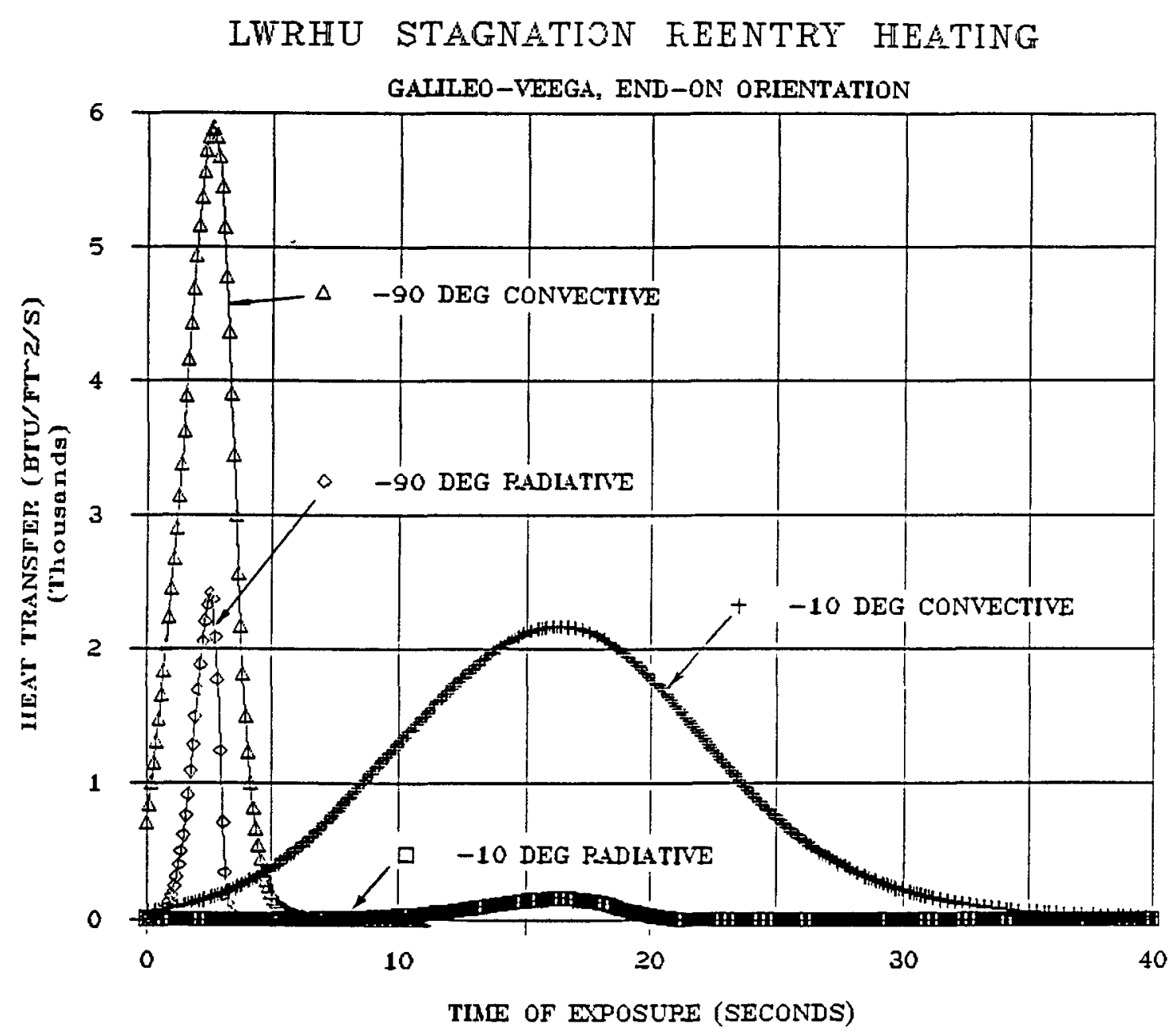

Fig. 25 LWRHU stagnation reentry heating. 


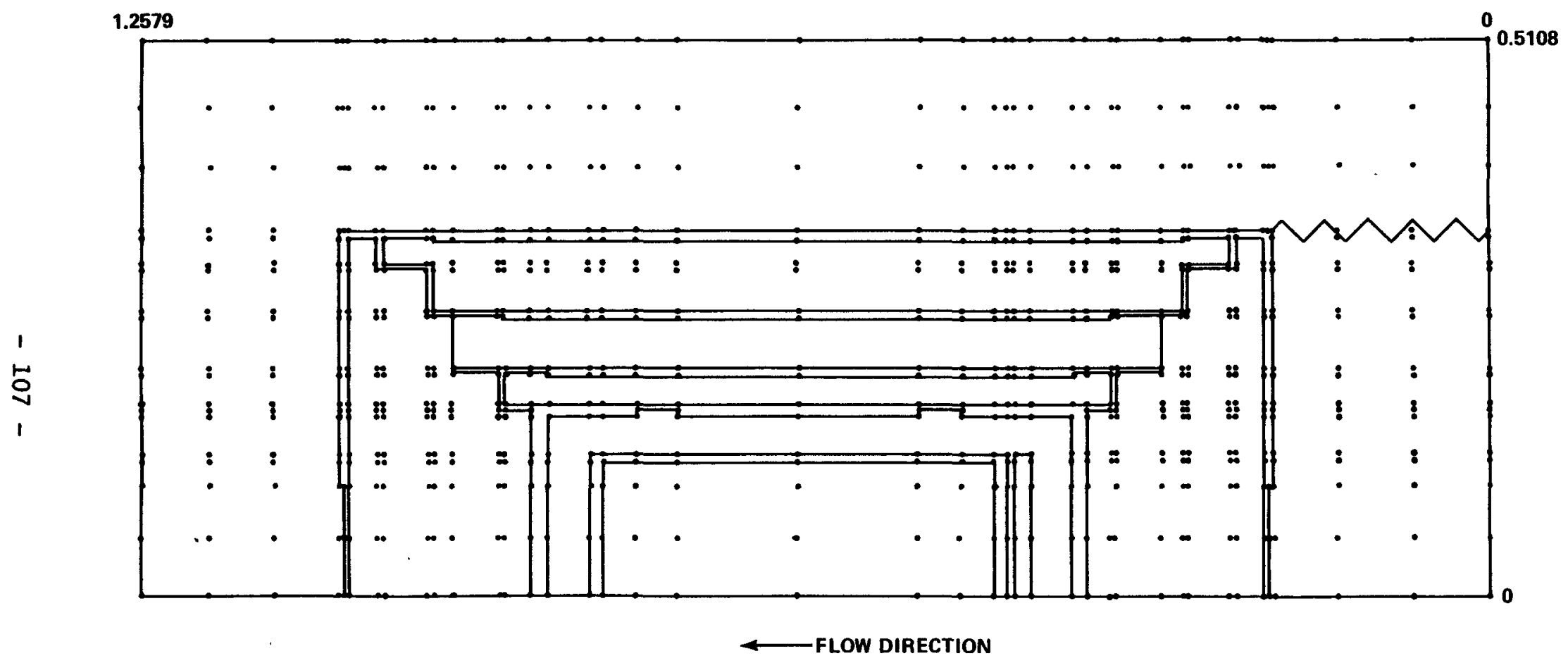

Fig. 26 Sketch of the end-on LWRHU thermal model which shows the 762 nodal points. Dimensions are in inches in this particular drawing. 


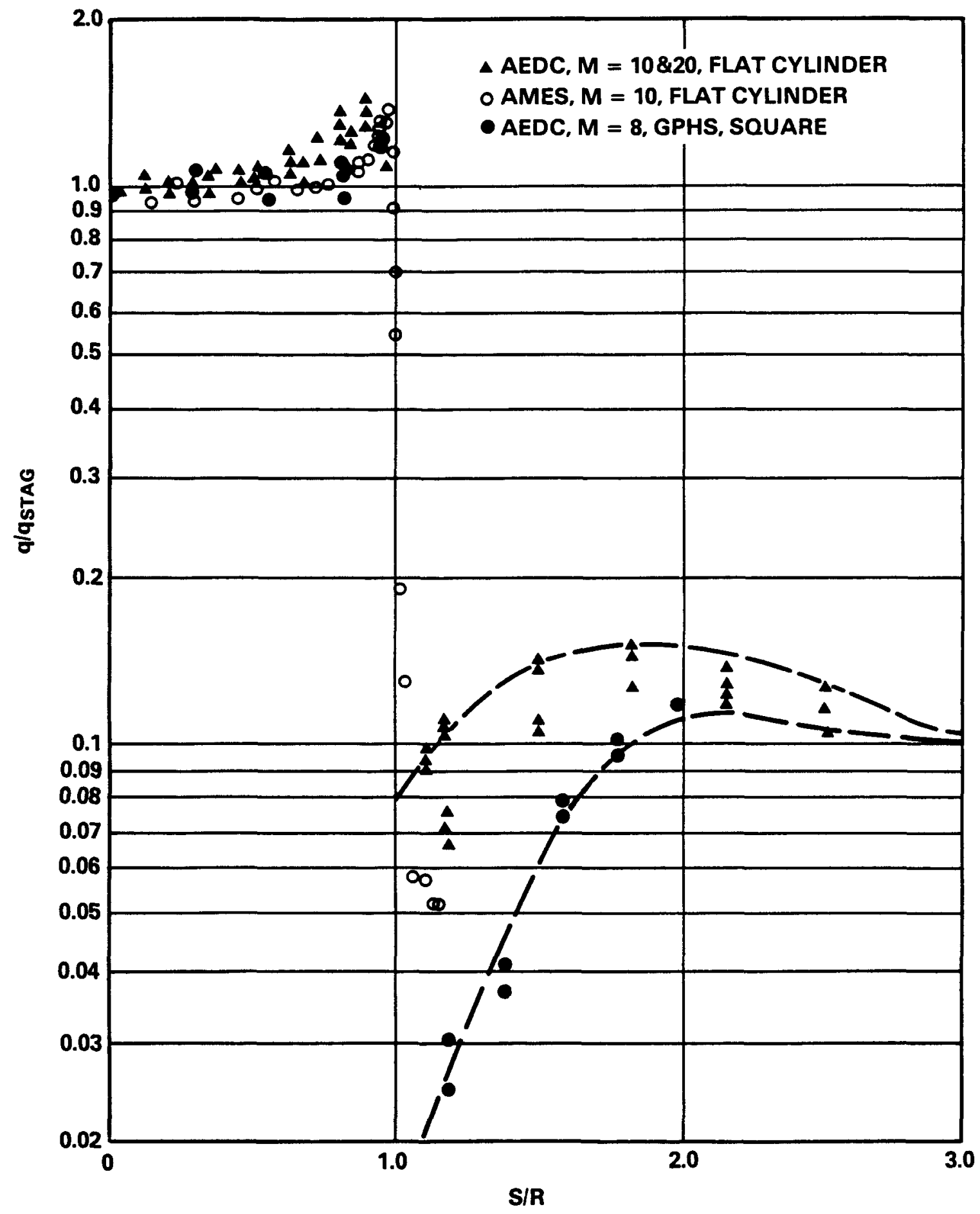

Fig. 27 Heating boundary conditions used in the APL analyses. 


\section{LWRHU -90 DEG. END-ON REENTRY}

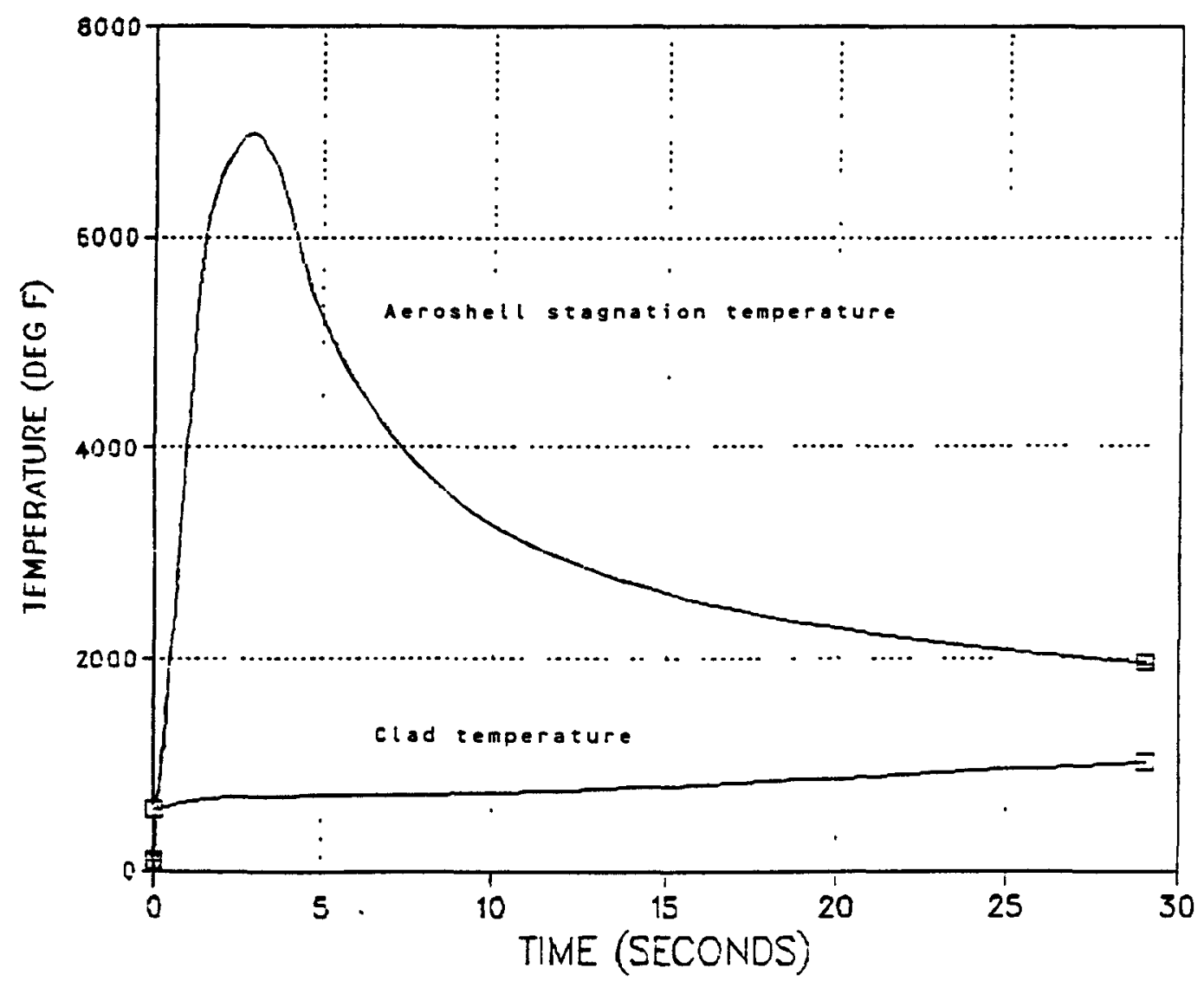

Fig. 28 LWRHU aeroshell windward end-face and clad temperature response. Galileo-VEEGA steep $\left(-90^{\circ}\right)$ end-on reentry orientation. 


\section{LWRHU -10 DEG. END-ON REENTRY}

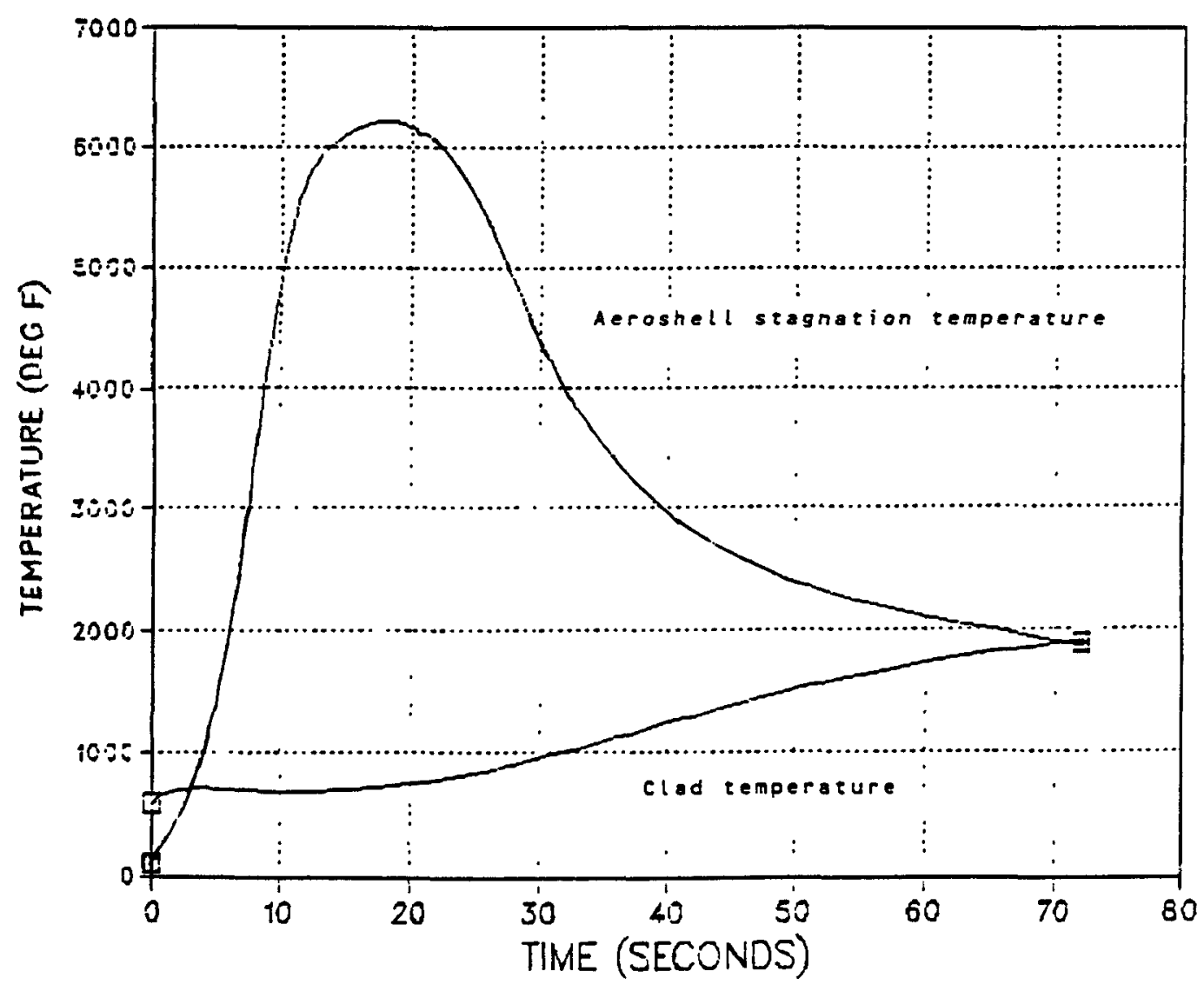

Fig. 29 LWRHU aeroshell windward end-face and clad temperature response. Galileo-VEEGA shallow $\left(-10^{\circ}\right)$ end-on reentry orientation. 


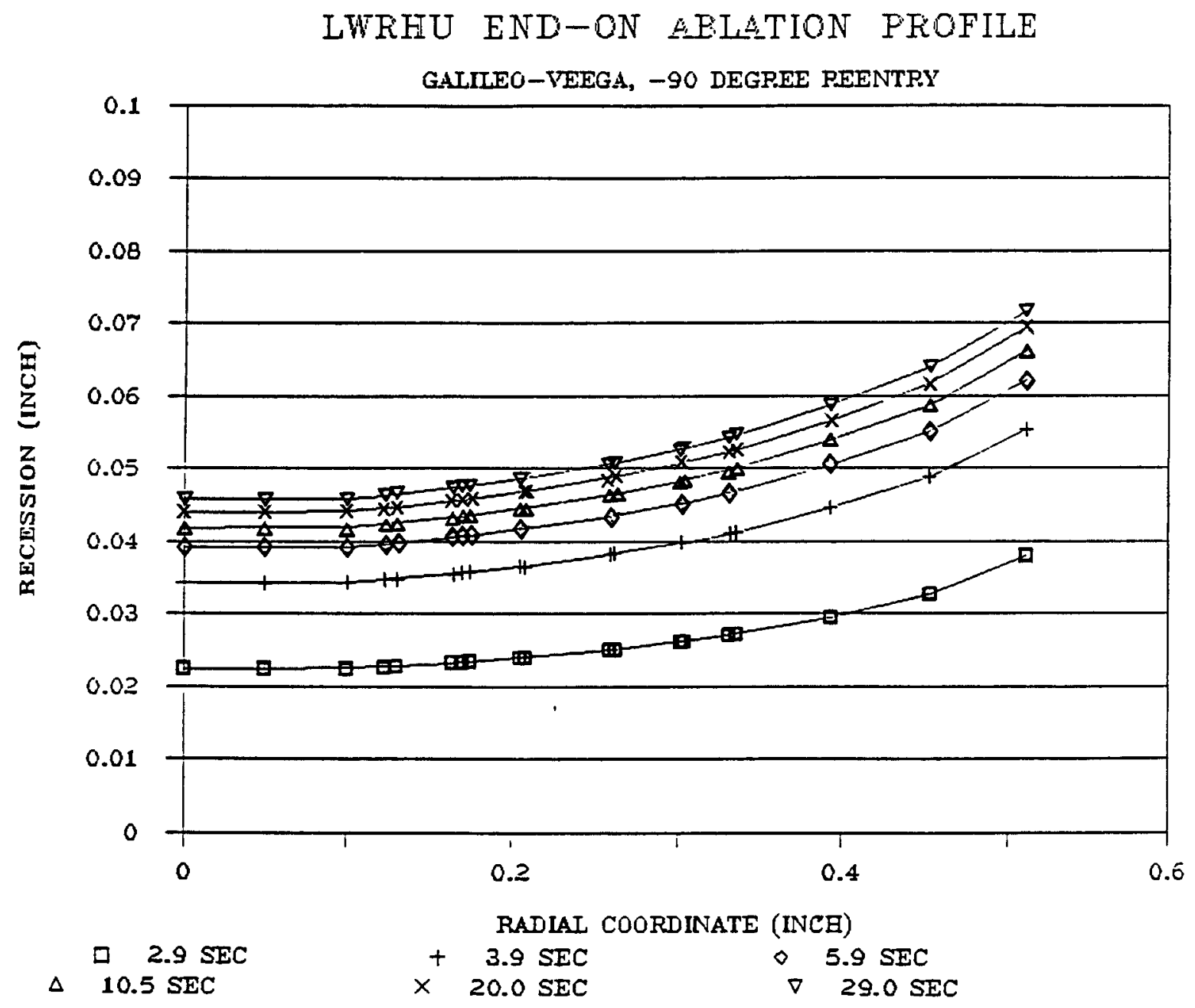

Fig. 30 LWRHU end-on ablation profile Galileo-VEEGA, $-90^{\circ}$ reentry. 


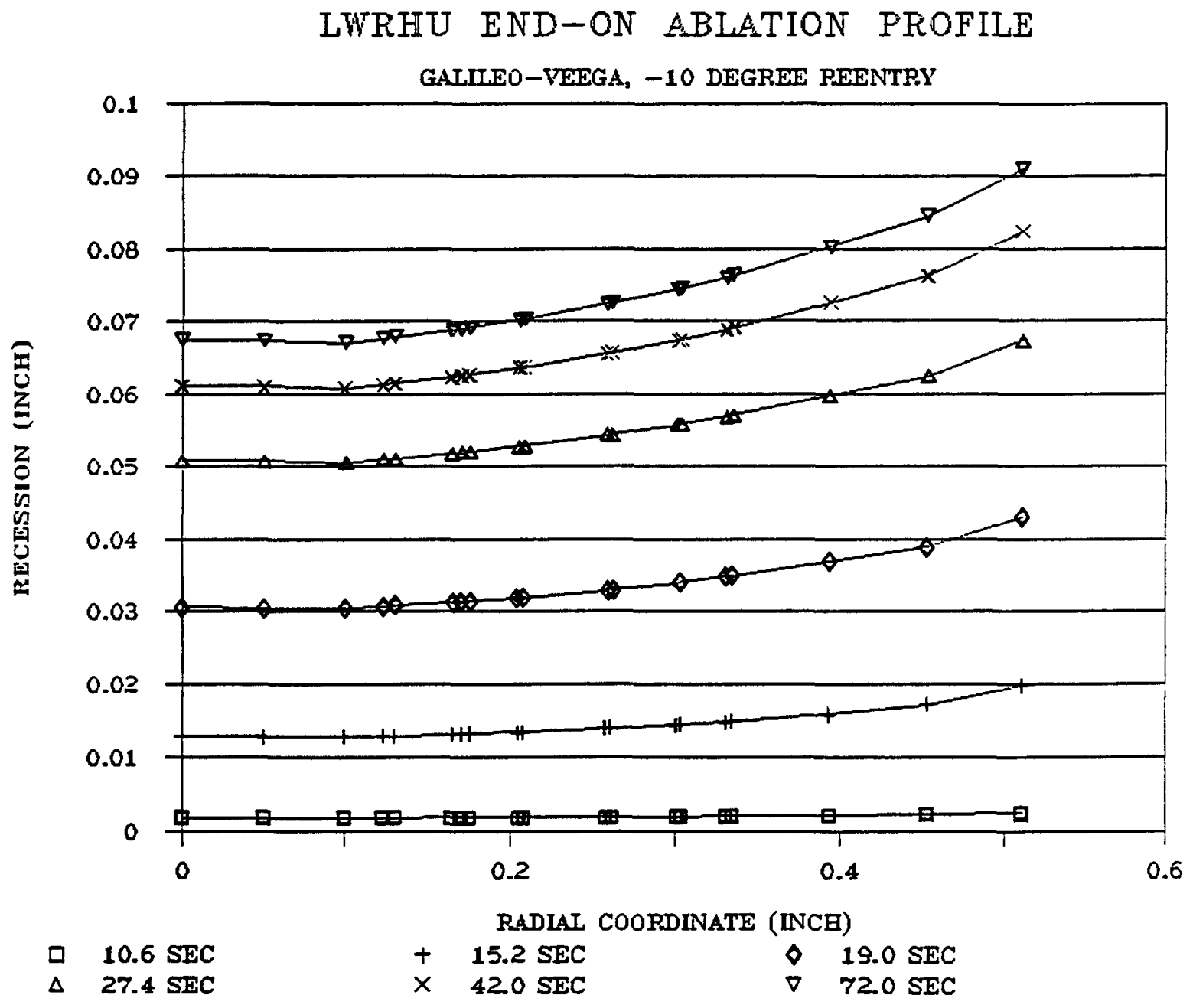

Fig. 31 LWRHU end-on ablation profile Galileo-VEEGA, $-10^{\circ}$ reentry. 


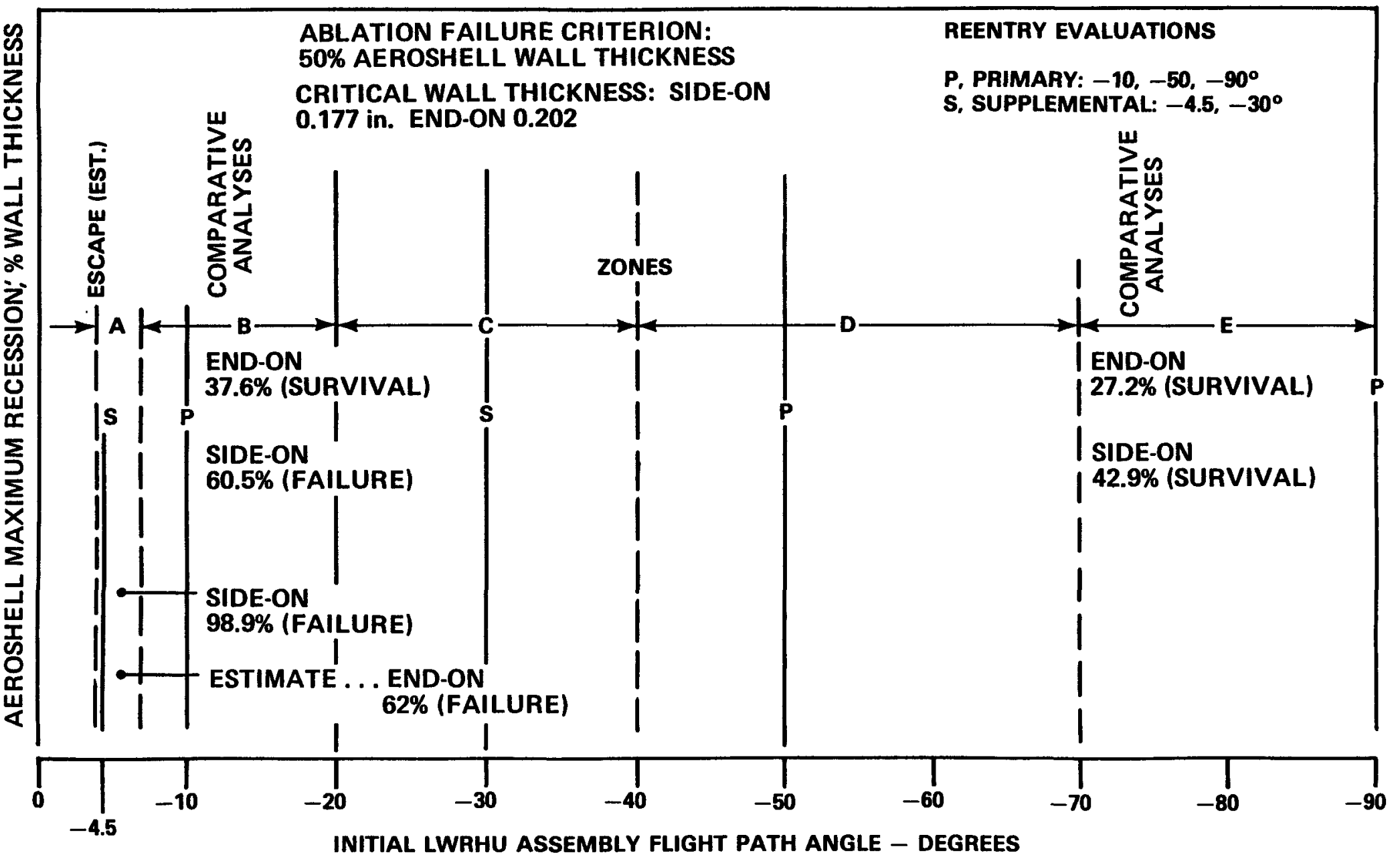

Fig. 32 Comparison of the aeroshell maximum recession predictions for side-on and end-on LWRHU assembly reentry configurations. 


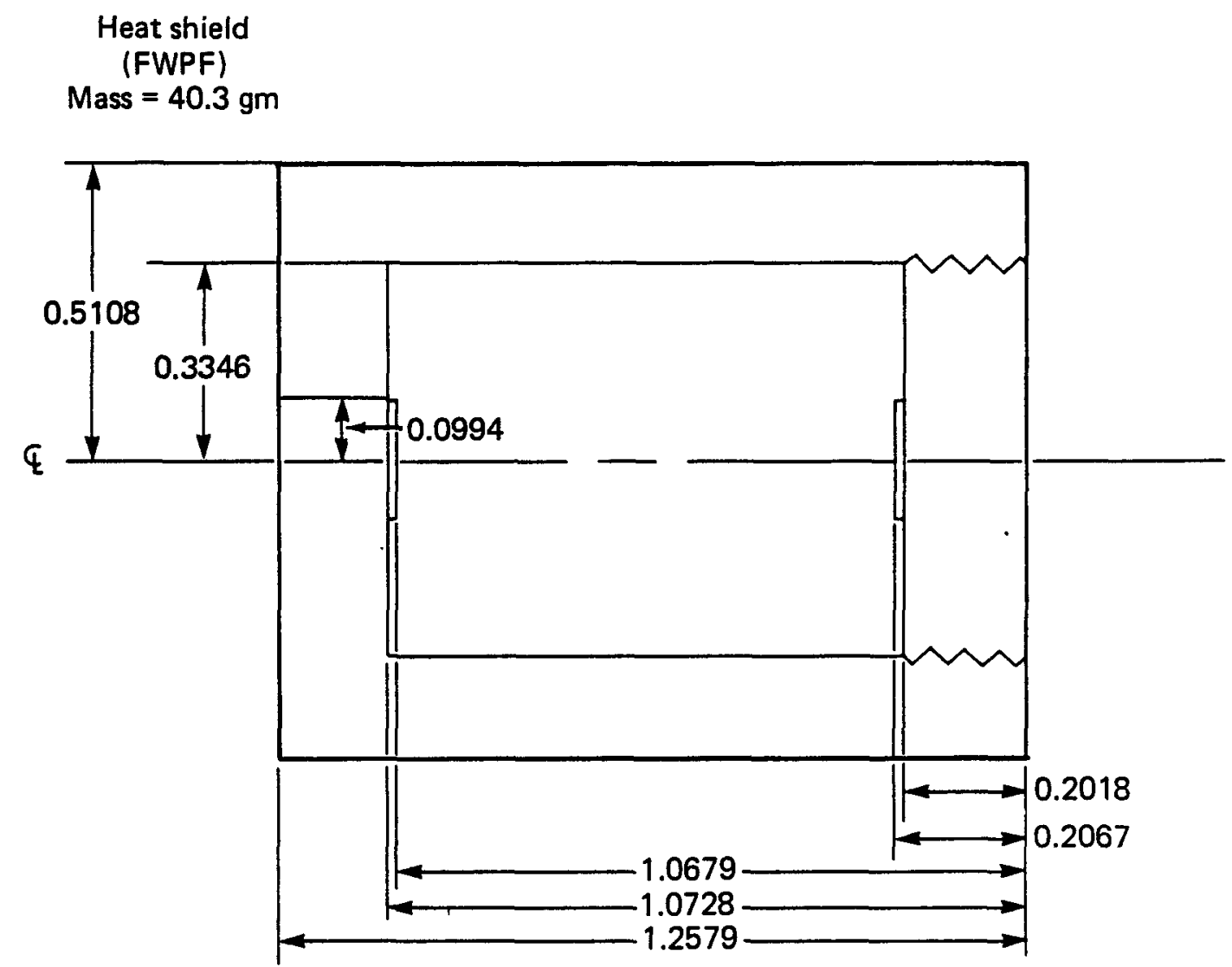

Fig. 33 LWRHU aeroshell, dimensions. 


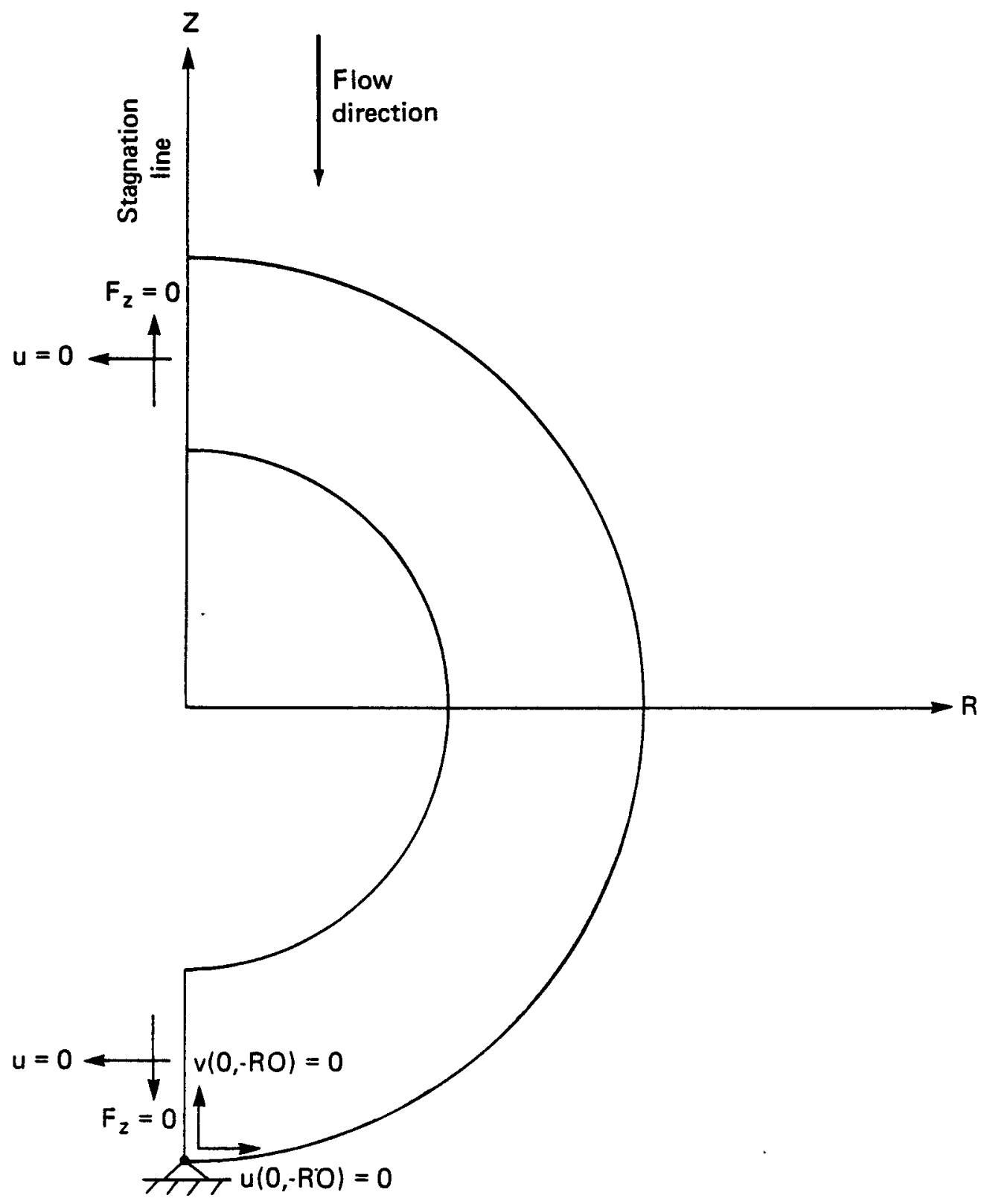

Fig. 34 Boundary conditions - side stable models. 


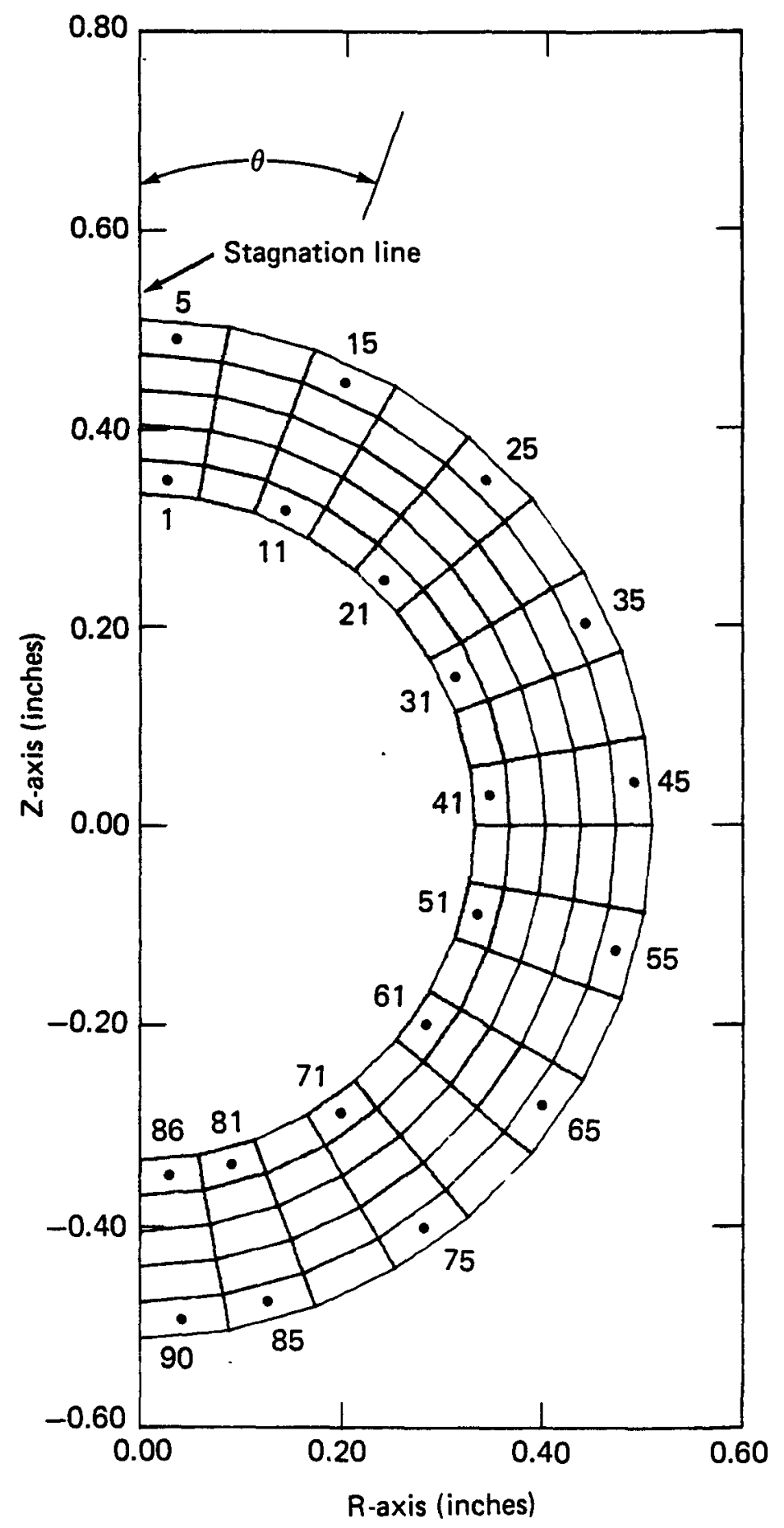

Fig. 35 Coarse mesh planar cross section model for side-on reentry calculations. 


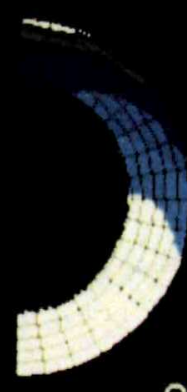

0.80 SEC

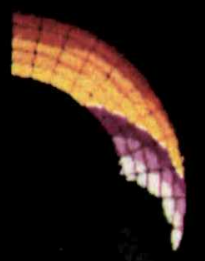

2.80 SEC

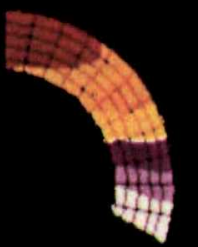

4.00 SEC
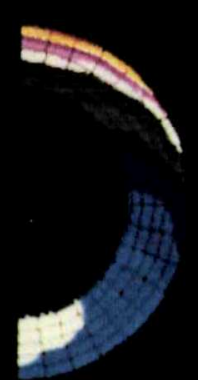

1.40 SEC

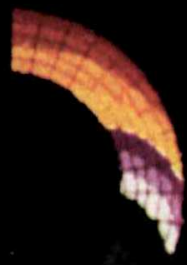

$3.20 \mathrm{SEC}$

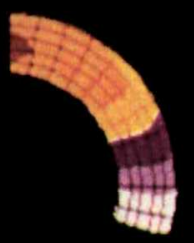

4. 40 SEC

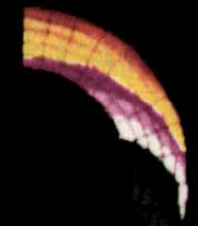

tet

2.40 SEC

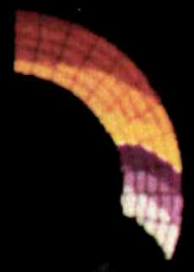

SIDE-ON STABLE 4451. VEEGA REENTRY

CONDITIONS

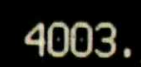

3555. 3107.

3.60 SEC VEL $=46750$ FPS 2659. GAMMA $=-90$ DEG

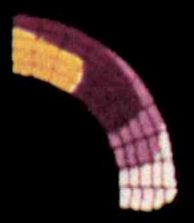
$\mathrm{ALT}=271000 \mathrm{FT}$ 2211. 1762. 1314. 866.

TEMPS IN DEG $F$ 5.60 SEC

Fig. 36 LWRHU nodal temperature distributions. 


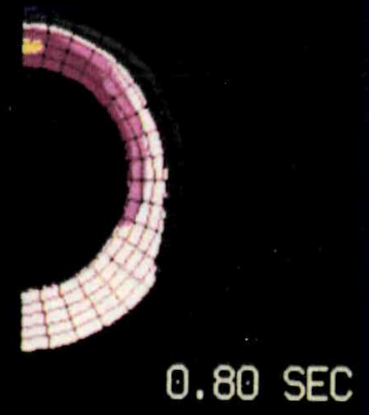

0.80 SEC

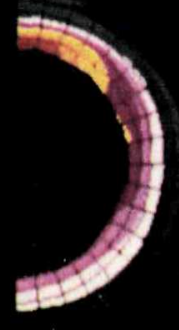

$1.40 \mathrm{SEC}$

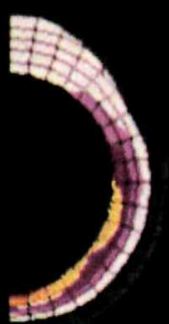

2.80 SEC

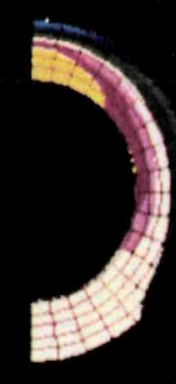

1.00 SEC

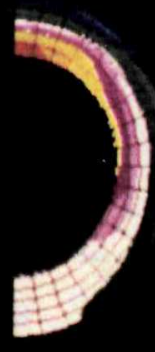

$1.20 \mathrm{SEC}$

COARSE MODEL HOOP STRESS

OISTRIBUTIONS

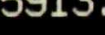

4997.

4080 .

3164.

2247.

1331.

SIDE-ON STABLE

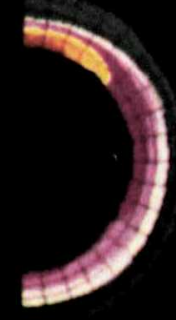

$1.60 \mathrm{SEC}$

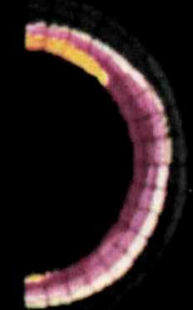

$1.80 \mathrm{SEC} \mathrm{VEL}=46750 \mathrm{FPS}_{-} 3252$

GAMA $=-90$ DEG -4168 . VEEGA REENTRY

CONDITIONS -1419 .

414.

-502 .
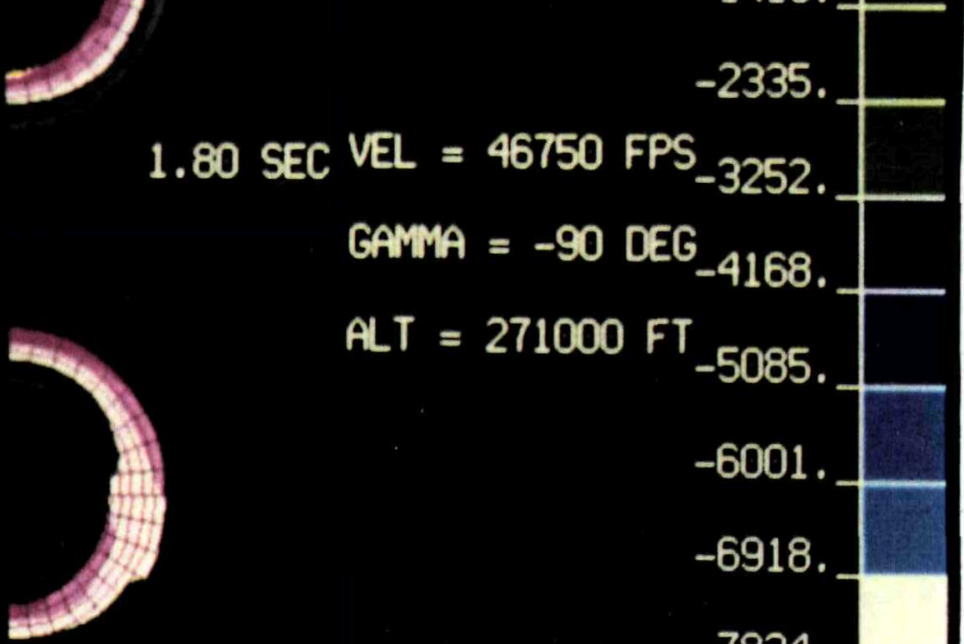

5.60 SEC

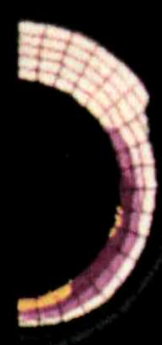

3.60 SEC
ALT $=271000 \mathrm{FT}$

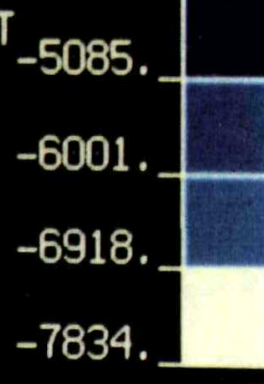

STRESSES IN PSI

Fig. 37 LWRHU coarse model hoop stress distributions. 


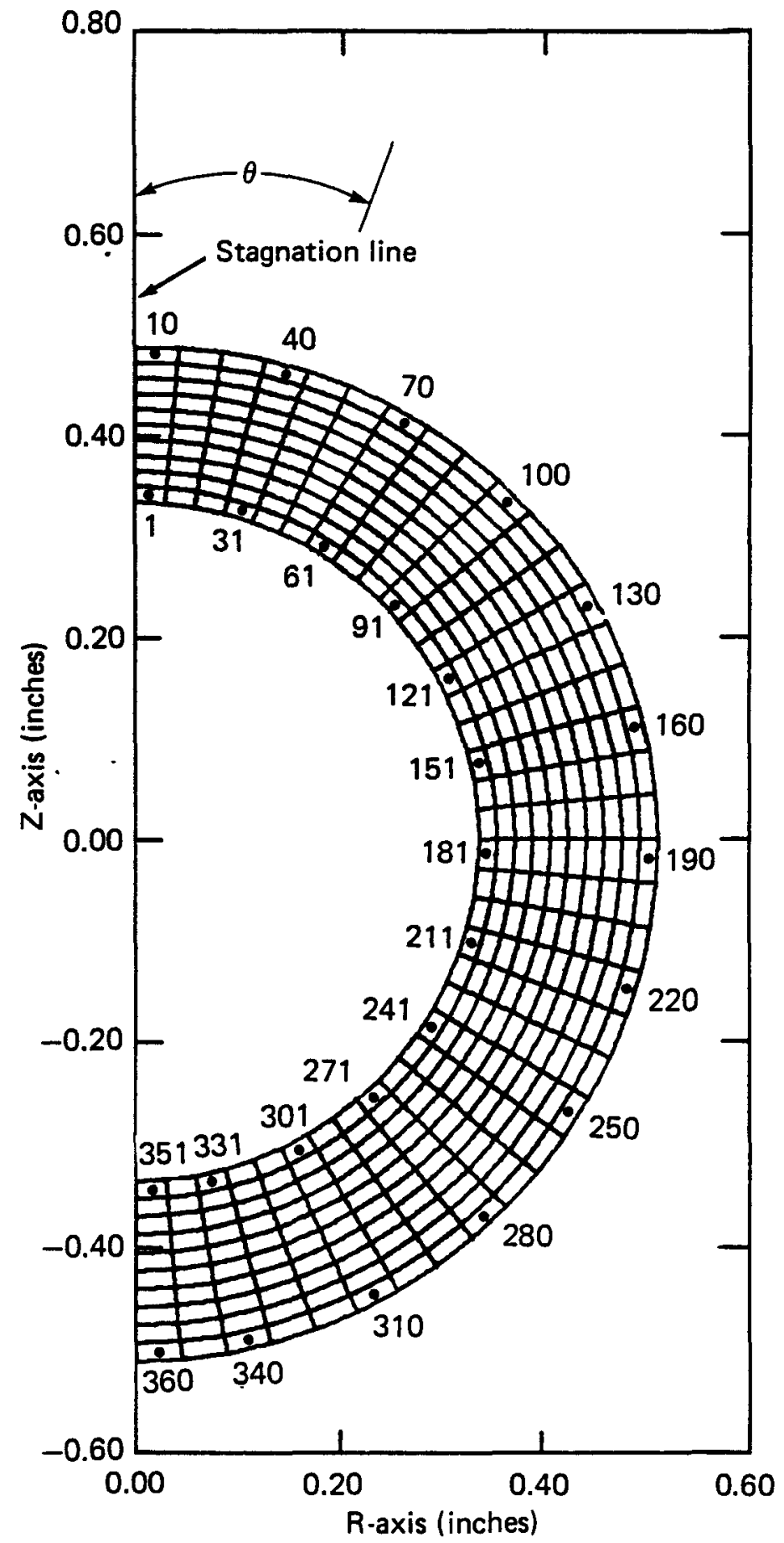

Fig. 38 Fine mesh planar cross section model for side-on reentry calculations. 


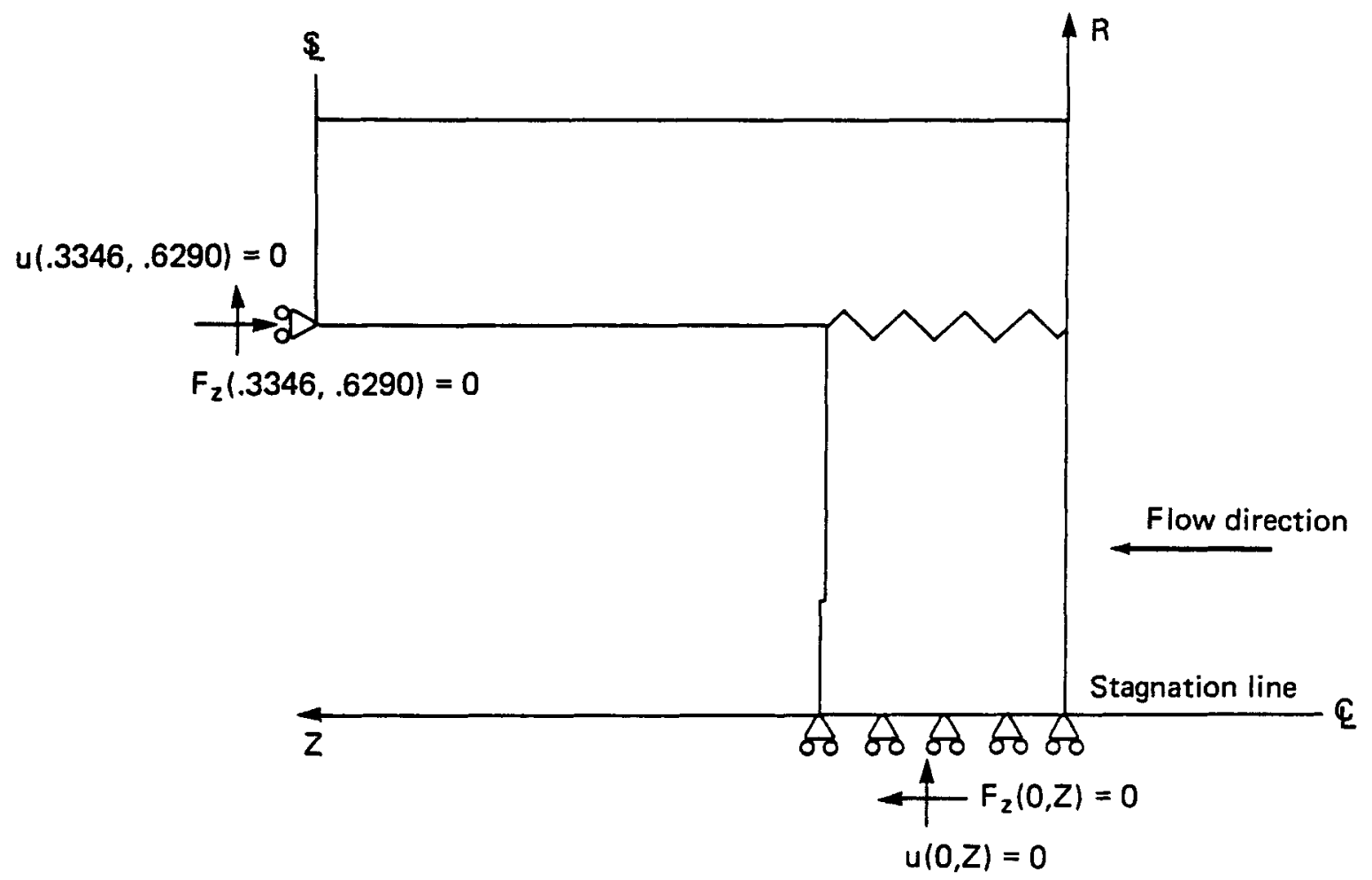

Fig. 39 Boundary conditions - end stable models. 


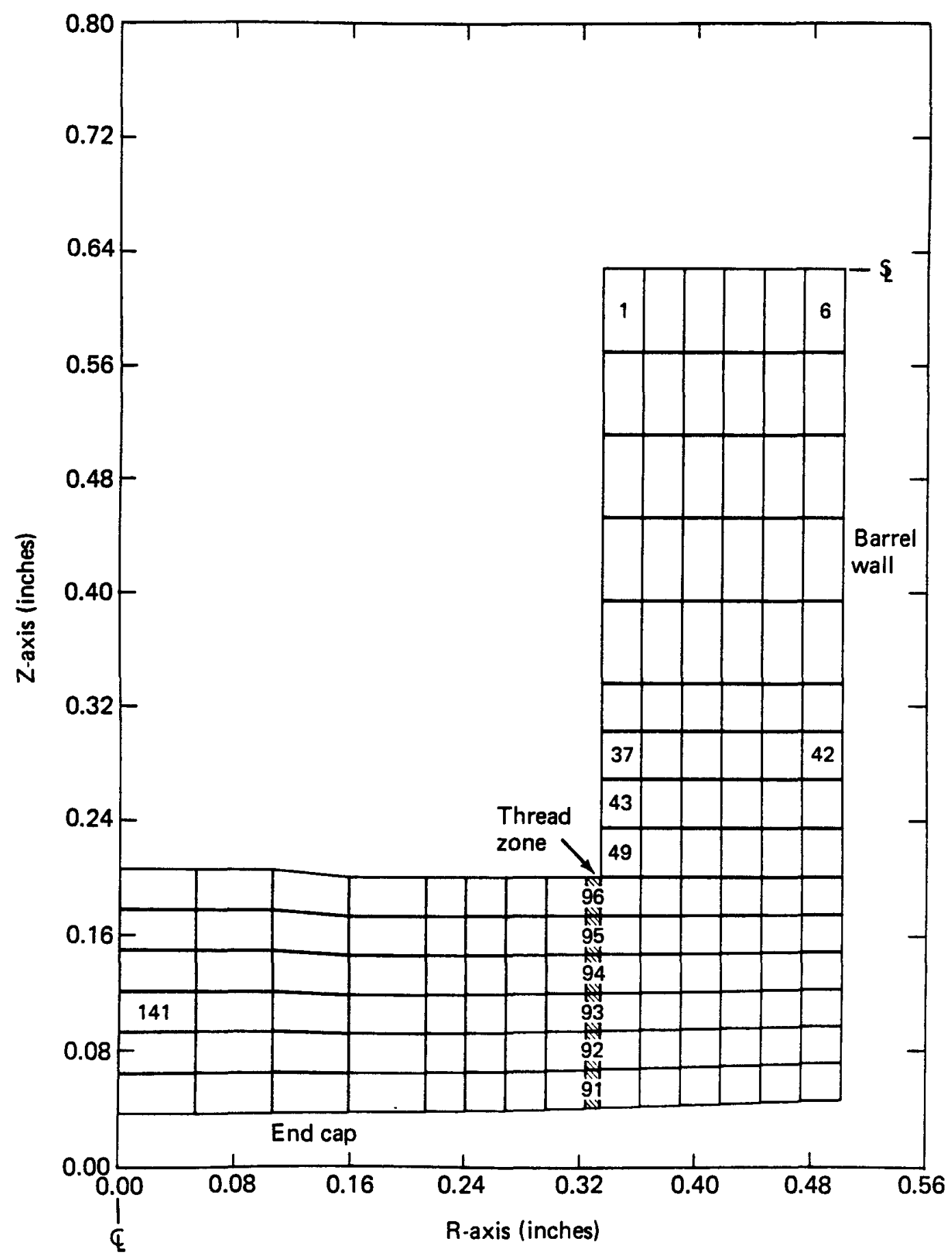

Fig. 40 Axisymmetric model for end-on reentry calculations. 


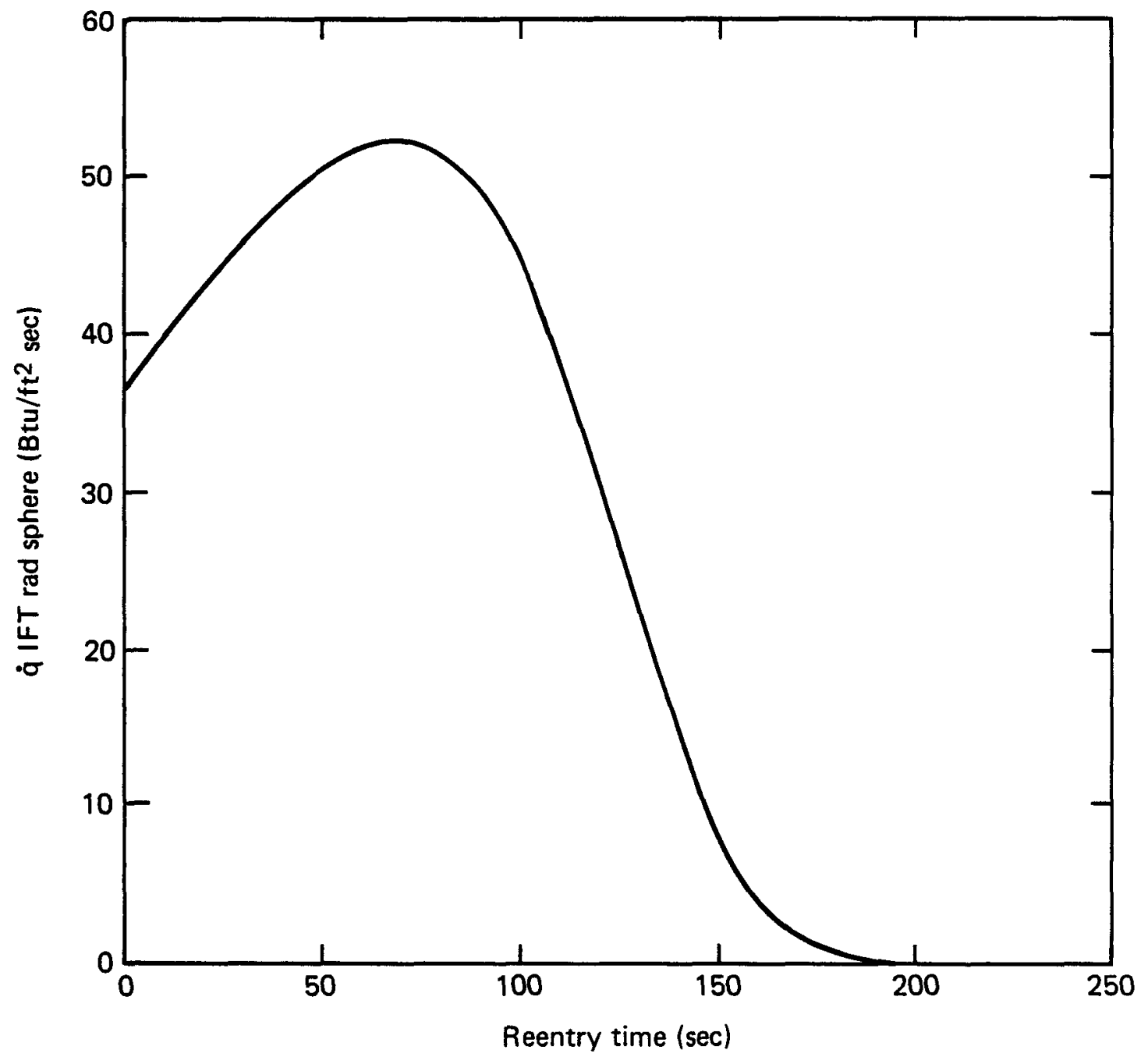

Fig. 41 Heat pulse of an orbital decay earth reentry for a LWRHU with the end-on orientation. 


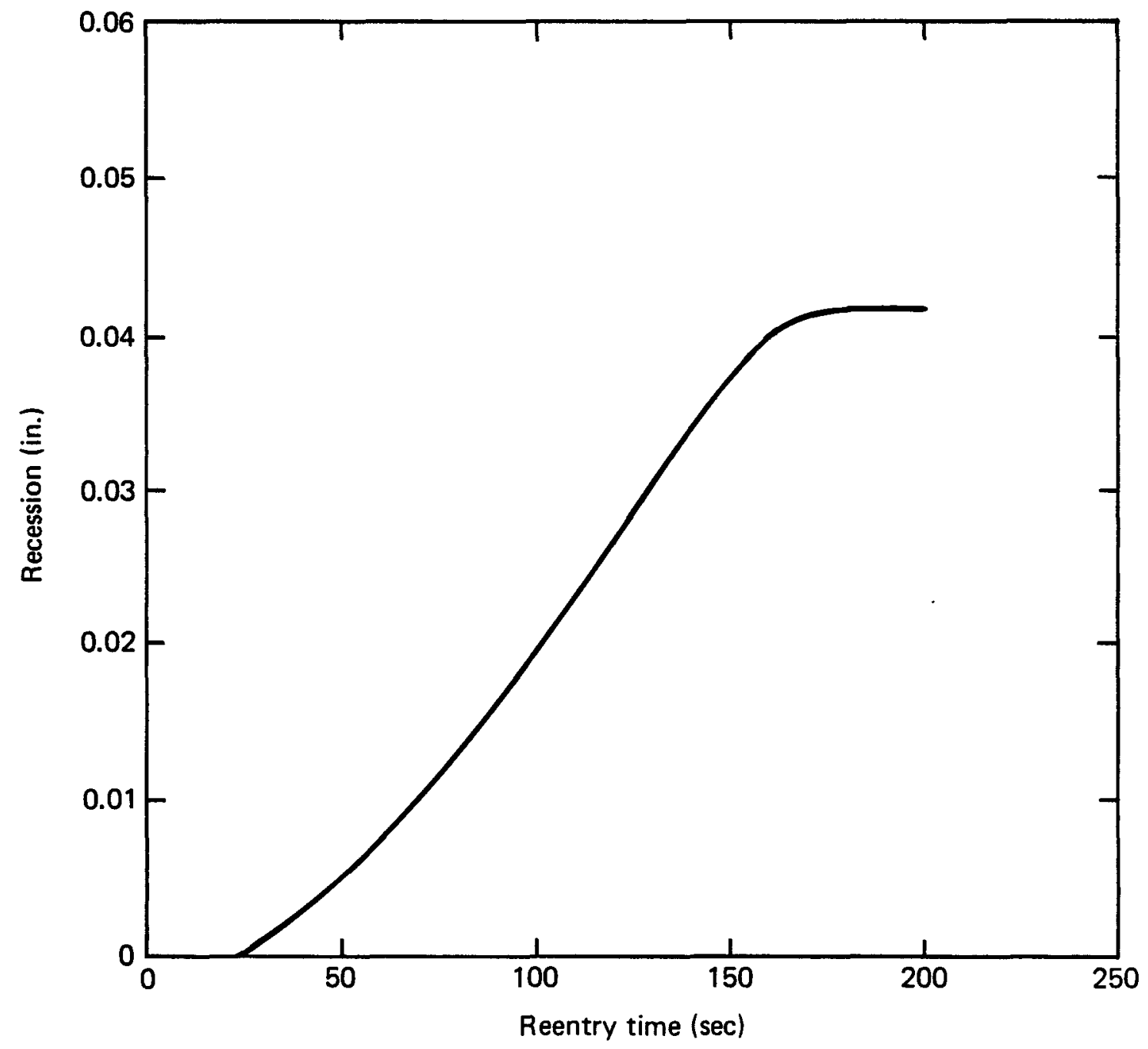

Fig. 42 Aeroshell recession at the stagnation point of the LWRHU for an orbital decay reentry with the end-on stable orientation. 


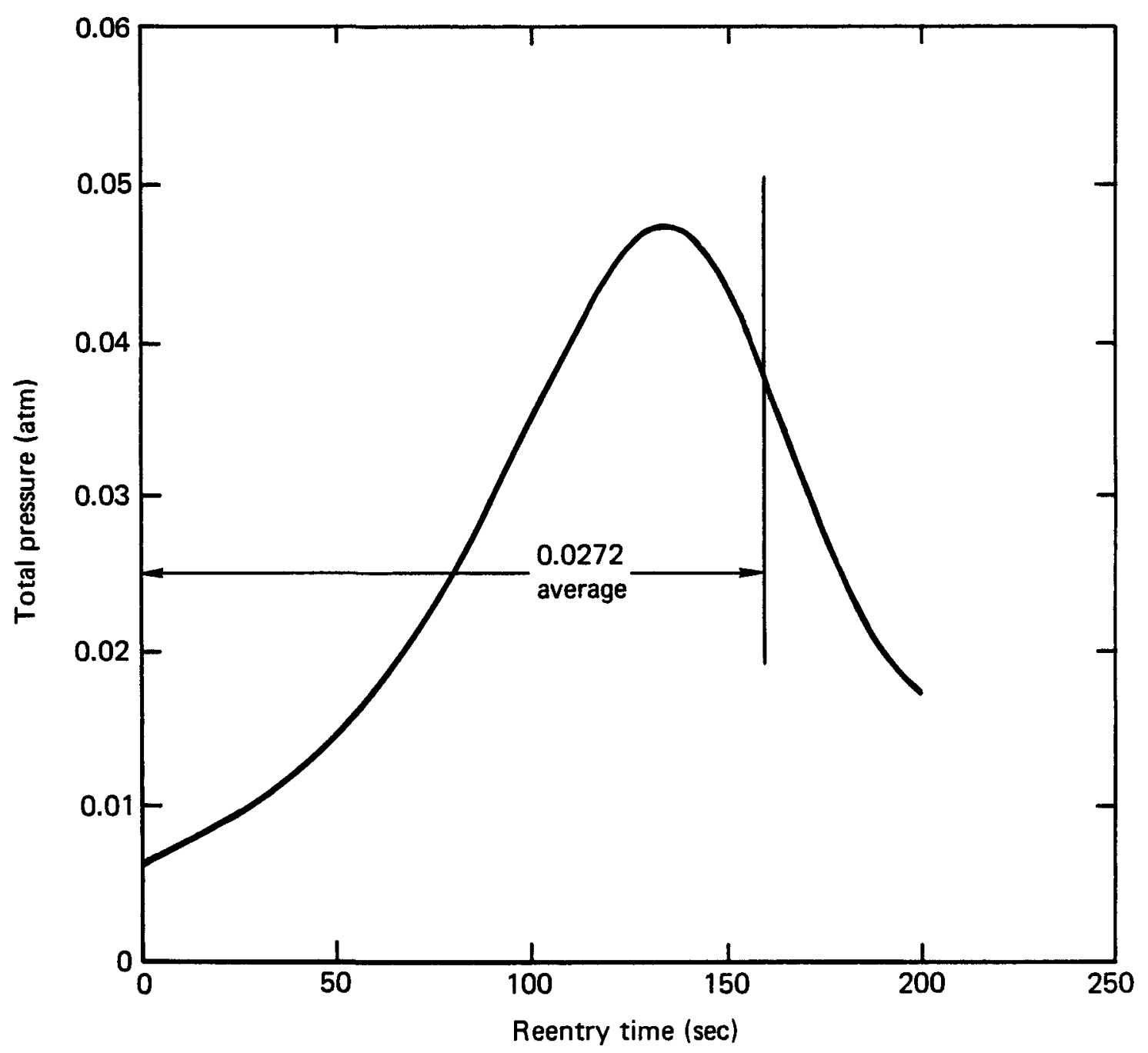

Fig. 43 Total pressure history of an orbital decay reentry for an LWRHU with the side-on stable orientation. 


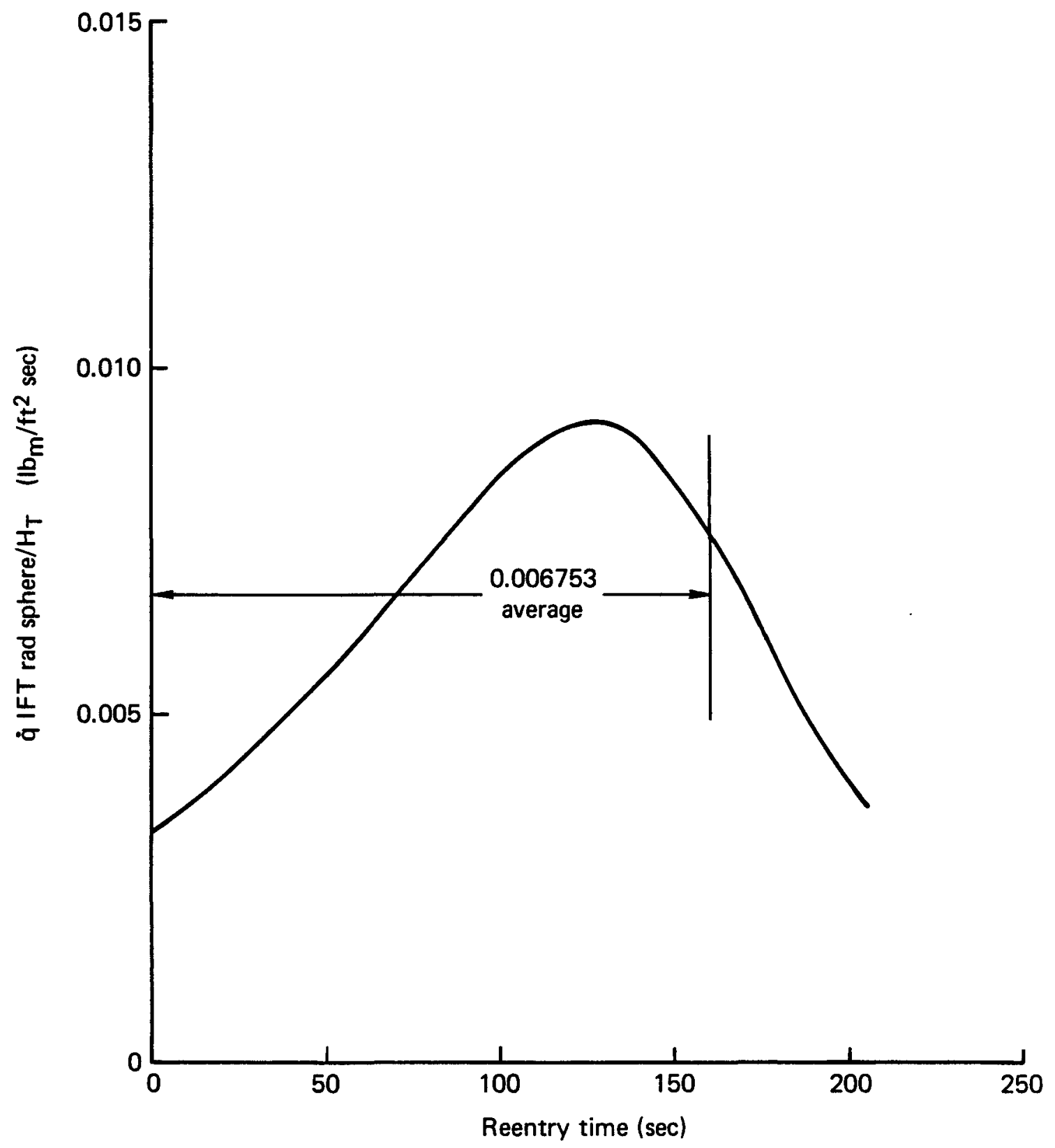

Fig. 44 Heat transfer parameter $\left(\dot{\mathrm{q}} / \mathrm{H}_{\mathrm{T}}\right)$ history for the LWRHU for an orbital decay reentry with the side-on stable orientation. 


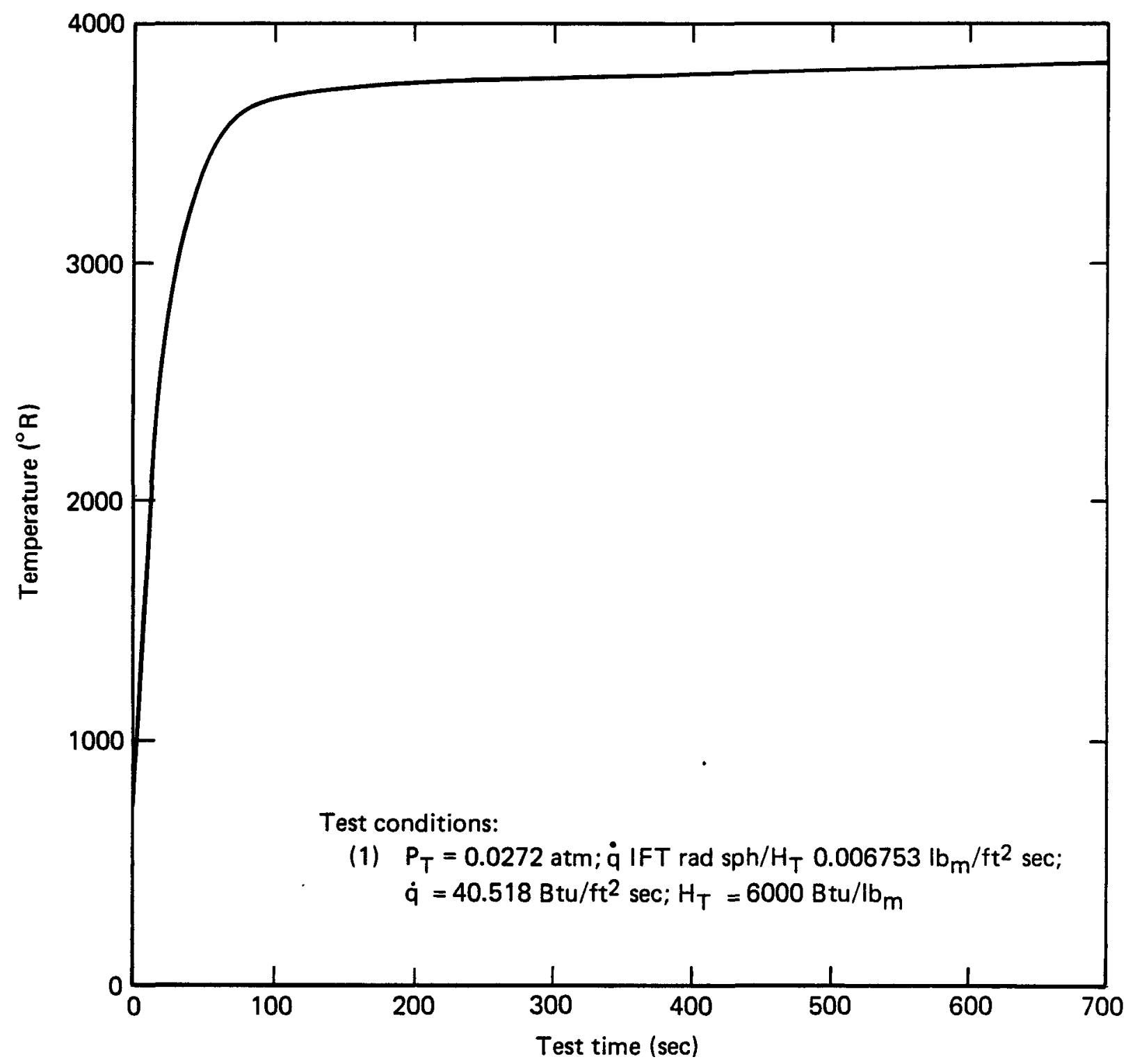

Fig. 45 End-on stagnation point temperature history for the LWRHU - $20 \mathrm{~mW}$., AHF, 12 in. nozzle. 


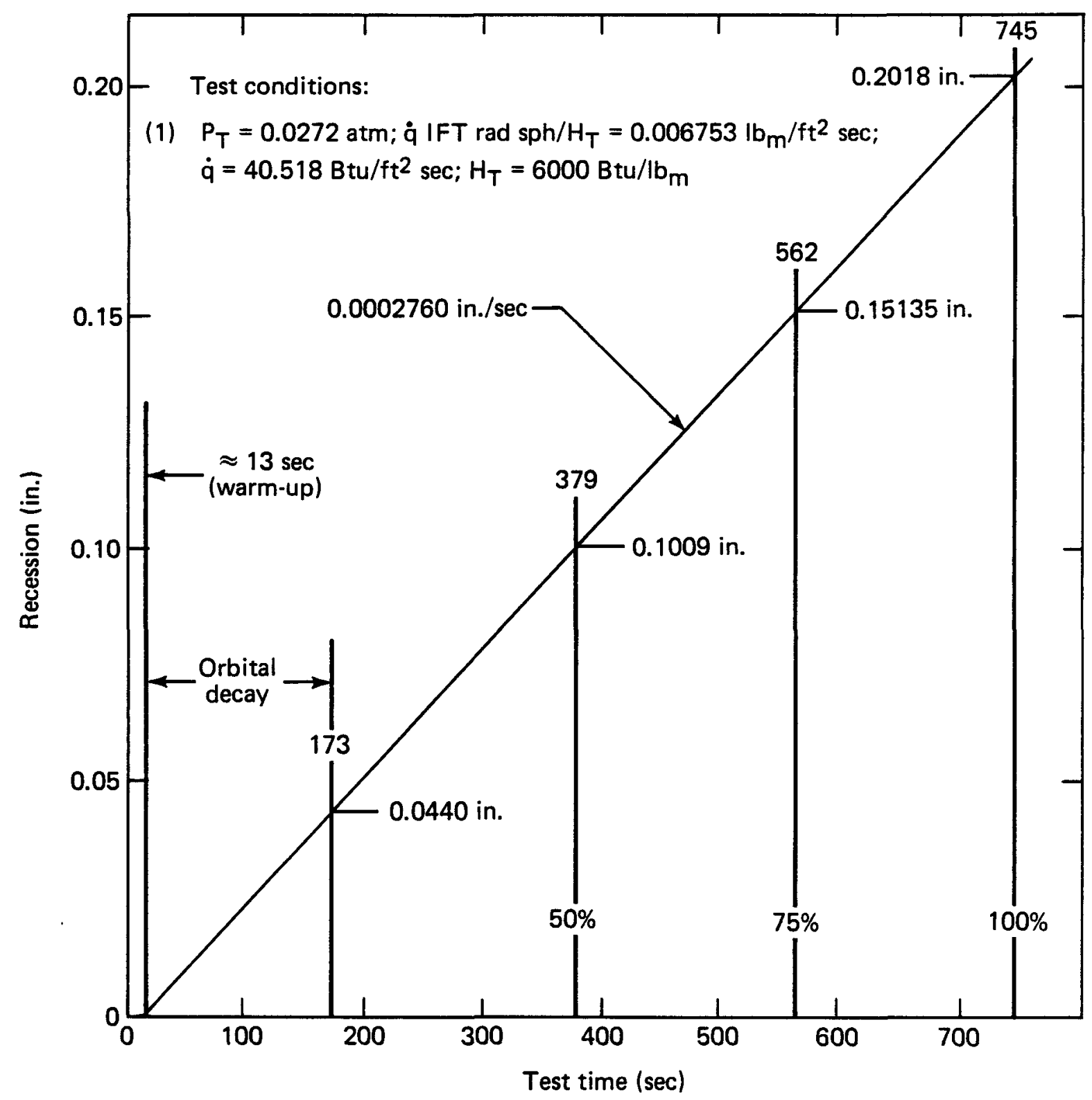

Fig. 46 Aeroshell recession at the end-on stagnation point for the LWRHU - $20 \mathrm{mw}$., AHF, 12 in. nozzle. 
Initial Distribution External to the Applied Physics Laboratory ANSP-M-19

The Aerospace Corporation

P. 0. Box 92957

Los Angeles, CA 90009

Battelle Columbus Laboratories

505 King Avenue

Columbus, $\mathrm{OH} 43201$

Fairchild Industries

20301 Century Bldg

Germantown, MD 20707

General Electric Company

Astro-Space Division

Building $B$

P. 0. Box 8555

Philadelphia, PA 19101

Jet Propulsion Laboratory

California Institute of Technology

4800 Oak Grove Drive

Pasadena, CA 91103

Los Alamos National Laboratory

P. O. Box 1663

Los Alamos, NM 87545

Monsanto Research Corp.

Mound Facility

P. 0. Box 32

Miamisburg, OH 45342

NASA/Headquarters

600 Independence A venue, S.W.

Washington, D.C. $2054^{\circ} 6$
W. H. Ailor

Mail Code M4/942

C. A. Alexander
R. F. Hartman, 29B12-KB
R. J. Hemler, 29B12-KB
J. R. Peterson, 29B12-KB
J. Loffreda, 20B41

A. D. McRonald, MS-157-102

S. E. Bronisz, MST-5/MS-G730

R. W. Zocher, CMB-11

E. Johnson, Bldg 88

W. R. Amos

D. McConnel1/EL

L. Grey/DS 
Initial Distribution External to the Applied Physics Laboratory (cont) ANSP $-M-19$

NASA/Langley Research Center

A. Wilhite, MS 365

Hampton, VA 23665

NUS Corporation

R. W. Englehart

910 Clopper Road

Gaithersburg, MD 20878

Teledyne Energy Systems

P. Dick

110 W. Timonium Road

Timonium, DM 21093

U. S. Department of Energy

office of Scientific and Technical

Information (OSTI)

Special Assistant for Reproduction and Processing

P. 0. Box 62

Oak Ridge, TN 37830

U. S. Department of Energy

Office of Special Applications

Washington, DC 20545

U. S. Department of Energy office of Procurement Operations 1000 Independence Avenue, S.W.

Washington, D.C. 20585
J. A. Turi, Director, NE-53

W. J. Barnett, NE-53

A. L. Mowery, NE-53

E. Mastal

Contracting officer, MA-453.2 
Internal Distribution:

P. T. Brenza

C. C. Chan

D. W. Conn

J. A. Ecker

E. F. Lucero

S. A. Lutz

E. T. Marley

R. P. Suess

L. B. Weckesser

F. B. Weiskopf

Archives/Files 